\title{
Bifurcation of the Periodic Orbits of Hamiltonian Systems: An Analysis using Normal Form Theory
}

D. A. Sadovskii

John B. Delos

William \& Mary, jbdelos@wm.edu

Follow this and additional works at: https://scholarworks.wm.edu/aspubs

Part of the Physics Commons

\section{Recommended Citation}

Sadovskii, D. A. and Delos, John B., Bifurcation of the Periodic Orbits of Hamiltonian Systems: An Analysis using Normal Form Theory (1996). Physical Review E, 54(2), 2033-2070.

https://doi.org/10.1103/PhysRevE.54.2033

This Article is brought to you for free and open access by the Arts and Sciences at W\&M ScholarWorks. It has been accepted for inclusion in Arts \& Sciences Articles by an authorized administrator of W\&M ScholarWorks. For more information, please contact scholarworks@wm.edu. 


\title{
Bifurcation of the periodic orbits of Hamiltonian systems: An analysis using normal form theory
}

\author{
D. A. Sadovskii ${ }^{1,2, *}$ and J. B. Delos ${ }^{1,2}$ \\ ${ }^{1}$ Department of Physics, College of William \& Mary, Williamsburg, Virginia 23187-8795 \\ ${ }^{2}$ Joint Institute for Laboratory Astrophysics, University of Colorado, Boulder, Colorado 80309-0440
}

(Received 26 October 1995)

\begin{abstract}
We develop an analytic technique to study the dynamics in the neighborhood of a periodic trajectory of a Hamiltonian system. The theory begins with Poincaré and Birkhoff; major modern contributions are due to Meyer, Arnol'd, and Deprit. The realization of the method relies on local Fourier-Taylor series expansions with numerically obtained coefficients. The procedure and machinery are presented in detail on the example of the "perpendicular" $(z=0)$ periodic trajectory of the diamagnetic Kepler problem. This simple one-parameter problem well exhibits the power of our technique. Thus, we obtain a precise analytic description of bifurcations observed by J.-M. Mao and J. B. Delos [Phys. Rev. A 45, 1746 (1992)] and explain the underlying dynamics and symmetries. [S1063-651X(96)10407-4]
\end{abstract}

PACS number(s): 03.20. $+\mathrm{i}, 02.30 . \mathrm{Hq}, 31.10 .+\mathrm{z}, 46.10 .+\mathrm{z}$

\section{PURPOSE. ORGANIZATION OF BIFURCATIONS OF PERIODIC ORBITS}

The modern study of periodic orbits (PO's) began with Poincaré [1] who realized that periodic solutions provide a route to the study of nonintegrable dynamics. Thus, the idea of chaos entered classical mechanics in close relation to periodic orbits. The concept of quantum chaos, the quantum analog of classical nonintegrability, was also formulated within a periodic orbit framework. Gutzwiller [2,3] showed how to use semiclassical approximations to calculate the density of states of a quantum system from PO's of the corresponding classical system. Similar semiclassical expressions were obtained for other observables, such as transition probabilities $[4,5]$.

A particularly rich structure has been studied for the states of the near-zero-energy Rydberg electron of the hydrogen atom in magnetic or electric fields [4-7]. In this case the large-scale structure of the absorption spectrum is formed by those short-time orbits of the electron that begin at and return to the nucleus. Many such recurrences have been clearly identified in the experimental data [8].

If fixed parameters of the system, such as energy or field strength, are made to vary, then the family of PO's changes quantitatively, but may also change qualitatively, as a result of bifurcations of orbits. For instance, as energy increases new periodic orbits can be created, and as order changes to chaos this results in a proliferation of PO's. In atoms in fields the new orbits are observed as new peaks that emerge in the recurrence spectrum when scaled energy changes (see Figs. 5 and 6 of Ref. [8]).

It follows that the mechanism of individual bifurcations of periodic orbits, and their patterns and sequences (their "organization"), are of fundamental interest for the study of the dynamics of nonintegrable classical systems and of their quantum analogs.

A bifurcation of a periodic orbit is associated with a quali-

\footnotetext{
*Present address: Université du Littoral, Quai Freycinet 1, Boite Postal 5526, 59379 Dunkerque Cedex 1, France.
}

tative change of the flow (or the phase portrait) in the immediate neighborhood of this orbit. Different types of bifurcations are characterized by different changes. The simplest way to observe these changes is to numerically generate the trajectories in the neighborhood of the given periodic orbit, and to plot a series of Poincaré surfaces of section for different values of the parameter.

For two degrees of freedom this approach gives a good idea of what happens, so it has been used extensively in application to concrete dynamical systems. It does not, however, address the question of why certain phenomena occur. Instead, it essentially produces "experimental" data-a result of a purely numerical experiment. Furthermore, in analyzing Poincaré surfaces of section we largely rely on the pattern-recognition ability of our eye-a wonderful device but, regrettably, a helpless one for plots of dimension higher than 2 or 3 . This limits the applications to two degrees of freedom, where in fact almost all of such work has been done.

The main purpose of this paper is to present a more general and appropriate strategy of attacking the problem. "Normal form theory" is a perturbation theory that combines with the principles of bifurcation theory and gives an approximate description of classical motion near a periodic orbit and of how that motion changes as the parameters of the system are made to vary. At its lowest level of approximation, normal form theory leads to the theorem of Meyer [9], which asserts that periodic orbits typically bifurcate in just five ways depending on the period-multiplication factor. We show that normal form theory can be used as a consistent quantitative theory and that at higher levels of approximation (with more terms) this theory describes not only bifurcations of the orbit itself but also bifurcations of other orbits nearby.

In particular, normal form theory provides a logical foundation for the observation that bifurcations of periodic orbits can occur in organized sequences [10]. For example, looking at the diamagnetic Kepler problem (DKP) defined in Appendix C, Shaw [11] and Mao and Delos ([6] Sec. V C 3) were studying how the new PO that they call "pac-man" was created near the perpendicular orbit. They saw a period-4 bifurcation that had the expected "four-island-chain" pattern 


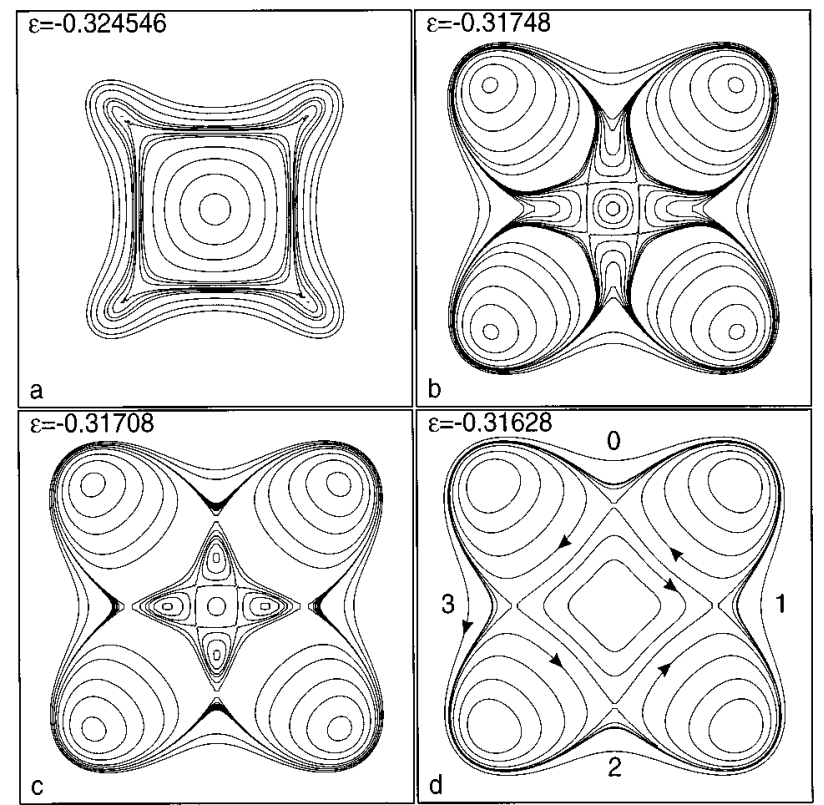

FIG. 1. "Organized" one-parameter bifurcations displayed by the normal form near the period-4 bifurcation of perpendicular orbit (contours are not drawn equidistantly).

(these terms will be explained in Sec. II B). However, "much more" was observed in the surfaces of section [Figs. 9(a), 9(b), and 9(c) of Ref. [6], and our Fig. 1). (1) A stableunstable pair of nearby period-4 orbits was created by a "saddle-node" bifurcation. (2) A second such pair was created in a similar fashion. (3) The separatrices rearranged into two concentric four-island chains. (4) Finally the actual period-4 bifurcation of Meyer's classification occurred: the inner chain shrank and collapsed onto the perpendicular orbit, leaving only the outer chain, whose $\mathrm{X}$ points correspond to the "pac-man" orbit. All these observations came out of careful examination of many numerical calculations; Shaw, Mao, and Delos (SMD) could neither anticipate nor explain these sequences of events. We use the term "organization" when we refer to such sequences of bifurcations.

What does normal form theory give us? Consider the function

$$
f_{\varepsilon}(\rho, \varphi)=\left(\varepsilon_{0}-\varepsilon\right) \frac{\rho^{2}}{2}-(1+\alpha+\cos k \varphi)\left(\frac{\rho^{4}}{4}-\frac{\rho^{6}}{6}\right),
$$

with $\rho$ and $\varphi$ polar coordinates on the plane, the order of the resonance $k=4, \alpha$ a fixed positive constant, and $\varepsilon$ a variable parameter that passes through a fixed value $\varepsilon_{0}$. (a) The contours of (1) reproduce numerically generated surfaces of section. Thus our Fig. 1 is a contour plot of a function qualitatively equivalent to (1) and it is indistinguishable from Fig. 9 of Mao and Delos [6]. In particular we examine the stationary points of (1): the points where $\partial f_{\varepsilon}(\rho, \varphi) / \partial \rho$ $=\partial f_{\varepsilon}(\rho, \varphi) / \partial \varphi=0$. These stationary points of (1) correspond to periodic orbits (fixed points of the Poincare map) found in Ref. [6]. As $\varepsilon$ varies, these stationary points are created and destroyed in an orderly sequence of events, precisely corresponding to that in [6]. (b) Normal form theory gives a systematic algorithm for constructing such functions from the exact total Hamilton function of the system. These functions constitute reduced effective Hamilton functions near a particular periodic orbit of the system. The function in Fig. 1 has been obtained as a normal form. All searching and experimentation with surfaces of section are thereby reduced to examination of contour plots and stationary points of simple functions.

Other bifurcations that SMD studied for the perpendicular orbit ([6], Fig. 11) proved to have a similar organization. Normal form theory provides an explanation: it shows that for all bifurcations of the perpendicular orbit above a certain scaled energy, the normal forms are qualitatively equivalent to (1) with $k=4,5,6, \ldots$ Specifically, the fourth- and sixthpower radial terms have alternating signs, and higher terms are small enough that they cause no qualitative changes in the vicinity. This is sufficient to guarantee the presence of just such an organized sequence of bifurcations.

SMD also found that one of the bifurcations of the perpendicular orbit seemed not to occur through such an organized sequence. Again normal form theory gives an explanation-at low scaled energies the alternation of signs does not occur in the normal form, so the complicated sequence is not present.

Normal form theory is also a natural and direct way to deal with a priori symmetries of a physical system, which can have important consequences for bifurcations of periodic orbits. In the case with symmetry Meyer's generic classification has to be modified to account for the bifurcations that are actually observed ([12], Appendix B of [6]). In normal form theory, a priori symmetries become explicitly built into the normal form, and they combine with additional symmetries that are induced by resonances. This leads to a complete classification of bifurcations of periodic orbits with symmetry. We plan to present such a classification in a separate future paper.

Intriguing observations [6-8] and the lack of understanding of the observed phenomena have inspired our present work. The methods we use are well developed in the mathematical literature. Poincare laid the foundation of normal form theory in his dissertation, Dulac considered normal forms near resonances [13], and Birkhoff treated normal forms near periodic orbits as an important case of the general theory [14]. An efficient Lie transform algorithm is a recent vital contribution due to Deprit and others [15]. Two contemporary sources, one by Meyer and Hall [16], and the other by Arnol'd [17], contain many original contributions to the use of normal forms in qualitative analysis of bifurcations. These books guided us well through the whole theory and their influence is invaluable.

In this paper we review these theories, and adapt them for our purpose. More important, we compose them into a unified, consistent procedure that can be used in a variety of applications. (To our knowledge, this paper is the first to carry through the whole process for a nontrivial periodic orbit.) The procedure is long, but ultimately rewarding. To make the procedure accessible to the general physics community, to show its realization in all details, and to demonstrate its usefulness we analyze the bifurcations of the perpendicular orbit of the diamagnetic Kepler problem. A brief summary of the results was published in [10], and we encourage the reader to return to that paper to keep the goals in mind. 
In Sec. II and in Appendix A, we present additional background information that underlies the theory. The details and implementation of the theory begin in Sec. III.

\section{INTRODUCTION. GENERIC BIFURCATIONS AND NORMAL FORMS}

At the heart of the theory is an intimate relationship between bifurcations of periodic orbits of Hamiltonian systems and bifurcations of stationary points of smooth functions of two variables. Let us introduce our subject by explaining that relationship (see also Appendix A).

\section{A. Generic bifurcations of stationary points of Morse functions}

Qualitative theory of generic functions, known in mathematics as Morse theory [18], is a basic theory that directly applies to generic Hamilton functions. The main ideas are intuitively simple and we survey them briefly.

\section{Morse functions on the line}

Consider a function $f_{\varepsilon}(q)$ that depends smoothly on a single variable $q$ and a single parameter $\varepsilon$. Let us say that $f_{\varepsilon}(q)$ changes qualitatively if and only if as parameter $\varepsilon$ varies, a new stationary point is created. This can occur only if for some $\varepsilon_{0}$ there is a degenerate stationary point $q_{0}$, such that both $f_{\varepsilon_{0}}\left(q_{0}\right)^{\prime}$ and $f_{\varepsilon_{0}}\left(q_{0}\right)^{\prime \prime}$ vanish. For example, consider the one-parameter family of functions

$$
f_{\varepsilon}(q)=\left(\varepsilon-\varepsilon_{0}\right) q+q^{3} .
$$

As $\varepsilon$ decreases through $\varepsilon_{0}$, this function changes qualitatively from one with no stationary points to one with a maximum and a minimum. This change is known as a "saddlenode" bifurcation or a "fold catastrophe." Moreover, a general smooth function having a degenerate critical point can be locally reexpressed in the form (2a) by a smooth change of coordinates, provided only that $f_{\varepsilon_{0}}\left(q_{0}\right)^{\prime \prime \prime}$ is nonzero. Therefore we say that the one-parameter family of functions (2a) is the normal form that represents the (only) generic creation of stationary points of functions on the line [19].

Now suppose that our functions are a priori restricted to be symmetric about $q=0$. The Taylor expansion can contain only even terms, and a bifurcation can only occur if the quadratic term vanishes, so the normal form

$$
f_{\varepsilon}(q)=\left(\varepsilon-\varepsilon_{0}\right) q^{2}+q^{4}+\cdots
$$

gives the typical (generic) bifurcation of symmetric functions on the line, called the "pitchfork" [20]: as $\varepsilon$ decreases through $\varepsilon_{0}$, the minimum at $q=0$ becomes a maximum, and two new minima are created.

If a symmetric function changes qualitatively near $q_{0} \neq 0$ we still expect the case (2a) - the only difference is that at the same time exactly the same bifurcation occurs near $-q_{0}$. Consider, for example, the function

$$
f_{\varepsilon}(q)=\varepsilon q^{2} / 2-q^{4} / 4+q^{6} / 6+\cdots,
$$

illustrated in Fig. 2. For $\varepsilon$ large and positive, there is a single

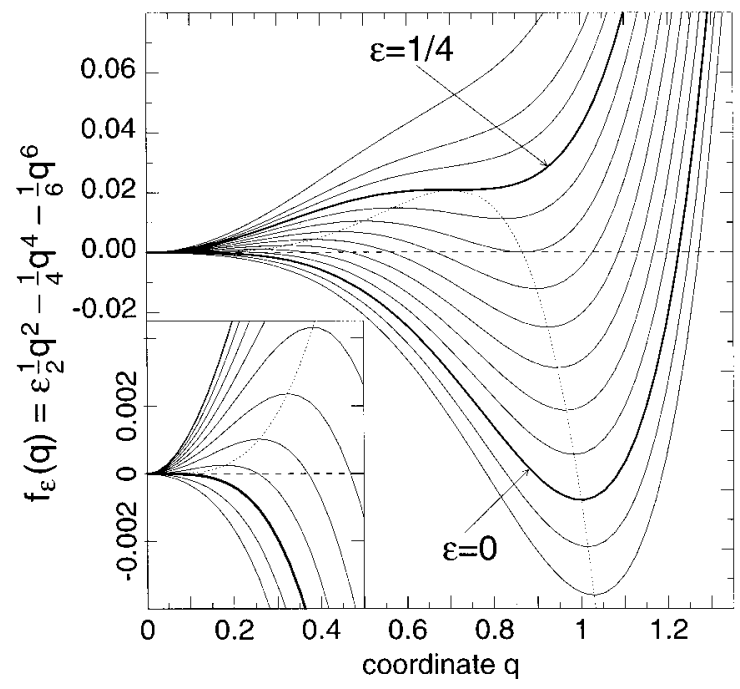

FIG. 2. "Organized" one-parameter bifurcations of even functions on the line exemplified by Eq. (2c); dotted line gives the position of stationary points.

stationary point at $q=0$. As $\varepsilon$ decreases through $1 / 4$, a new local maximum $(\max )$ and minimum $(\min )$ are created at a critical point at $q_{0}=1 / \sqrt{2}$, and they move apart as $\varepsilon$ continues to decrease. This is again a "saddle-node" bifurcation, with cubic normal form (2a). Due to the symmetry of (2c) about $q=0$ a twin max-min pair appears at the same time at $q_{0}^{\prime}=-1 / \sqrt{2}$. As $\varepsilon$ decreases through zero, the twin maxima move to the origin, "collide" with it, and disappear, leaving the origin a local max for negative $\varepsilon$ [" [pitchfork" bifurcation, with normal form (2b)].

We further note that the two bifurcations are organized: they form a sequence of two events caused by a monotonic change of a single parameter. In one-parameter theory this phenomenon of organization is not generic. However, if high-order terms in (2c) are sufficiently small, so that cutting them off gives a qualitatively correct behavior of $f_{\varepsilon}$ in the neighborhood of 0 , such organization can be common: it takes place if the fourth- and sixth-power terms in (2c) have opposite signs.

Function (1), which described the bifurcations of PO's near the perpendicular orbit, is essentially (2c) with an angular modulation. As stressed in Sec. I, the organization of bifurcations of stationary points manifested in function (1) has been observed for many bifurcations of periodic orbits $[6,10,11,21]$.

\section{Morse functions on the plane}

The theory for the planar case is central to our study. The stationary points

$$
\left(q_{0}, p_{0}\right): \frac{\partial f_{\varepsilon}\left(q_{0}, p_{0}\right)}{\partial q}=\frac{\partial f_{\varepsilon}\left(q_{0}, p_{0}\right)}{\partial p}=0
$$

of generic (Morse) functions $f_{\varepsilon}(q, p)$ are such that the Hessian matrix is nonsingular:

$$
\Omega_{\varepsilon}=\left(\frac{\partial^{2} f_{\varepsilon}}{\partial(q, p)^{2}}\right), \quad \operatorname{det} \Omega_{\varepsilon}\left(q_{0}, p_{0}\right) \neq 0 .
$$


Whenever (3b) holds, the stationary point (3a) is isolated from any other stationary point (is nondegenerate), and it persists (can be continued) over a range of $\varepsilon$.

What are the typical qualitative changes in this case? The simple answer has been given by Zhilinskií and Pavlichenkov [22]. The idea remains the same as in the case of the line, but on the plane there are more possible symmetry properties to consider, such as

$$
f(q, p)=f(\mathrm{R}(q, p)), \quad \mathrm{R}=C_{1}, C_{2}, C_{3}, C_{4}, C_{k>4},
$$

the rotations of the plane by angle $2 \pi / k$. The corresponding normal forms are shown in Table I [23].

In this table, the $C_{1}$ (no symmetry) and $C_{2}$ (inversion) normal forms are the obvious two-dimensional generalizations of Eqs. (2a) and (2b). For $C_{1}$, we get the saddle-node bifurcation: an $\mathrm{X}$ point (saddle) and an $\mathrm{O}$ point (max or min) are created where there was no stationary point before. For $C_{2}$ symmetry, there are two possible types of pitchfork bifurcation: $\mathrm{O} \rightarrow \mathrm{X}$ with two new $\mathrm{O}$ 's created, or $\mathrm{X} \rightarrow \mathrm{O}$ with two new X's created [20].

To understand the higher symmetry cases, let us examine $k=4$ in more detail. Consider a function $f_{\varepsilon}(q, p)$ that must have $C_{4}$ symmetry about the origin. The Taylor expansion of $f_{\varepsilon}(q, p)$ is best expressed in polar coordinates $(\rho, \varphi)$, and, because of the imposed symmetry, this expansion can contain any power of $\rho^{2}$, and it can also contain $\rho^{2 i} \cos 4 j \varphi$ and $\rho^{2 i} \sin 4 j \varphi$, with $j$ any integer. However, to make $f_{\varepsilon}(q, p)$ smooth in $(q=\rho \sin \varphi, p=\rho \cos \varphi)$ at the origin $q=p=0$, we must have $2 i \geqslant 4 j$. If the coefficient of the quadratic term $\rho^{2}$ is nonzero, then the origin is an isolated stationary point, so a bifurcation can only occur if that coefficient passes through zero. Typically all other coefficients in this expansion will not become zero at the same time. Therefore, the generic representation (normal form) for bifurcations of $C_{4}$ functions is

$$
f_{\varepsilon}(q, p)=\varepsilon \rho^{2}+\frac{1}{4} a \rho^{4}+\frac{1}{4} b \rho^{4} \cos (4 \varphi)+\cdots .
$$

The structure of the contour plots of (5) depends on the relative magnitudes of the constants $a$ and $b$. If $|a|>|b|$, then (for $a>0$ ) when $\varepsilon>0$ the origin is an isolated min ( $O$ point in the contour plot). When $\varepsilon$ passes through zero and becomes negative, the origin becomes a max, and nearby there are four symmetrically placed mins separated by saddle points ( $\mathrm{X}$ points in the contour plots). We call this an "island chain" bifurcation.

The reader can now verify the following. (i) If $|b|>|a|$, the $C_{4}$ bifurcation (5) has a different structure: four saddles approach so that they collide with the origin when $\varepsilon=0$, and then reappear with a different orientation when $\varepsilon$ changes sign ("touch-and-go" bifurcation). (ii) For $C_{3}$ symmetry, the generic structure can only be "touch and go." (iii) For $C_{k}$ symmetry with $k \geqslant 5$, the only generic behavior is the "island chain." (Hint: compare the degree in $\rho$ of the main resonance term $\rho^{k} \cos k \varphi$ and of $\rho^{4}$.)

\section{B. Generic bifurcations of periodic orbits: Meyer's classification}

Meyer [9] has established a simple theorem, which we can state intuitively as follows. When we examine bifurca-
TABLE I. Generic one-parameter $C_{k}$-symmetric Morse functions $f_{\varepsilon}: R^{2} \rightarrow R$ and their bifurcations.

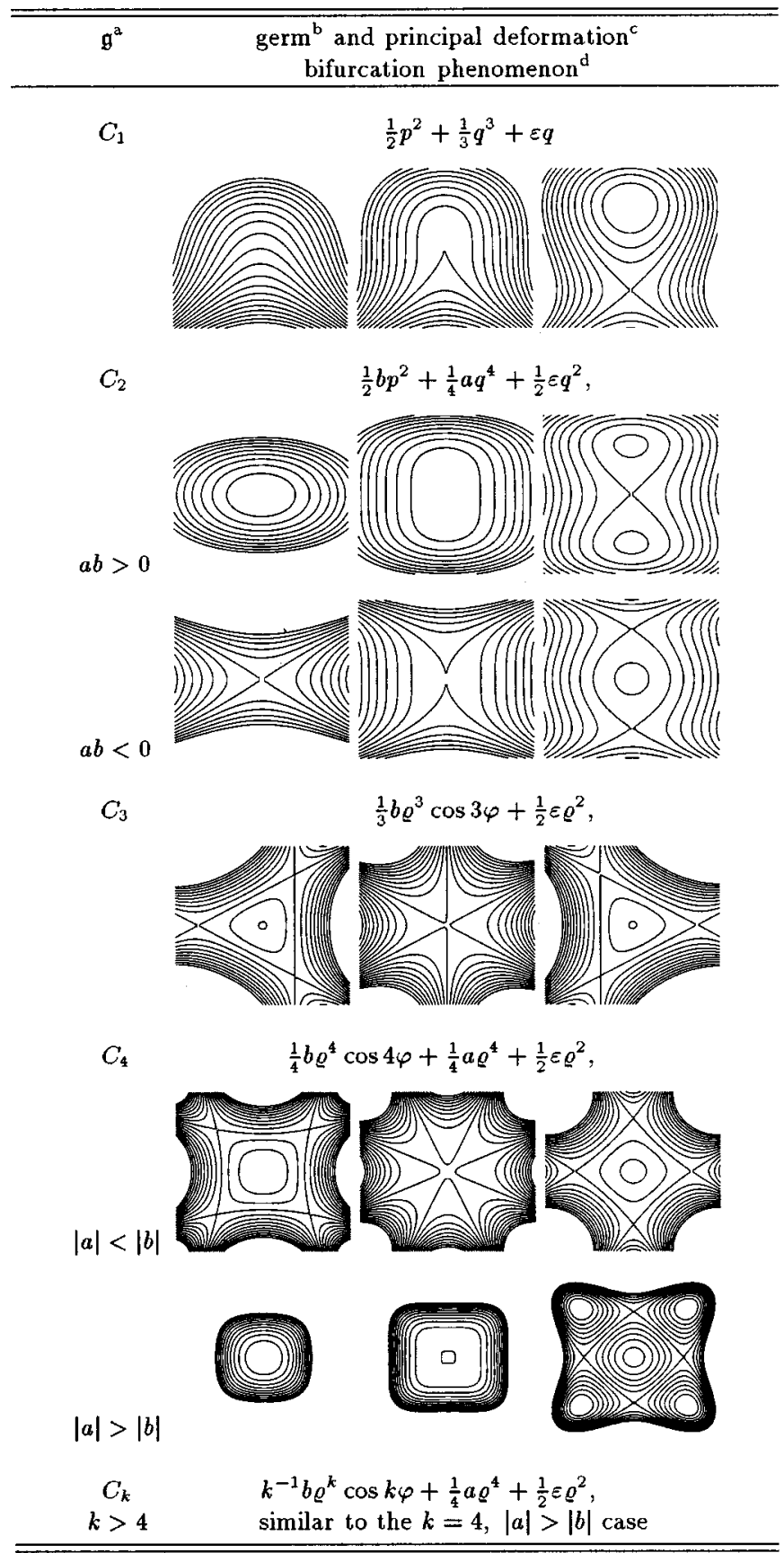

${ }^{a}$ Local symmetry in the neighborhood of the stationary point.

${ }^{\mathrm{b}} \mathrm{We}$ assume the critical value of parameter $\varepsilon_{0}=0$ so that the germ of the family corresponds to the expression in this column without the last term; $a \neq 0$ and $b \neq 0$.

${ }^{\mathrm{c}}(q, p)$ and $(\varrho, \varphi)$ are local rectangular and polar coordinates.

${ }^{\mathrm{d}}$ The middle contour plot corresponds to the critical value $\varepsilon_{0}$.

tions of periodic orbits of Hamiltonian systems resulting from the change of a single parameter, then the patterns that our eyes will see in a surface of section are the same patterns that are shown in Table I. A period- $k$ bifurcation of a periodic orbit looks like a $C_{k}$ bifurcation of stationary points of a smooth function of two variables.

To understand this correspondence, several points must be explained. (a) The theorem describes generic behavior- 
other things can happen, but they would be exceptional. (b) We need to know the analog of the Hessian condition: under what condition is a $\mathrm{PO}$ isolated in $(q, p)$ and continuable in $\varepsilon$ ? (c) Why is there such a close correspondence between bifurcations of PO's (which are special solutions to differential equations defined in a $2 N$-dimensional phase space) and bifurcations of stationary points of functions of only two variables? (d) How does symmetry enter? Nothing is assumed $a$ priori about symmetries of the Hamiltonian system; yet a period- $k$ bifurcation somehow manifests local $C_{k}$ symmetry. (e) What do we mean when we speak of "the patterns our eyes will see"? How complete is the correspondence between bifurcations of PO's and bifurcations of stationary points?

\section{Monodromy matrix and continuable orbits}

The linear stability of a periodic orbit $\Gamma_{\varepsilon}(t)$ is defined by the (eigenvalues of) monodromy matrix $M_{\varepsilon}$, the linear part of the Poincaré map:

$$
x \mapsto P_{\varepsilon}(x)=M_{\varepsilon} x+\cdots
$$

It can be obtained if we linearize equations of motion near $\Gamma_{\varepsilon}$ and integrate them over the period.

In $N$ degrees of freedom $M_{\varepsilon}$ is a symplectic matrix of dimension $2 N-2$; in particular $\operatorname{det} M=1$. It has $N-1$ pairs of reciprocal eigenvalues ([16], Chap. II C) $\lambda(\varepsilon)$, called multipliers, such that $\lambda_{1} \lambda_{2}=1$. For $N=2$ (two degrees of freedom) generic matrices $M$ have [24]

$$
\lambda_{1,2}=\exp ( \pm i \omega)
$$

with characteristic exponents $i \omega$ either real (hyperbolic or unstable case) or purely imaginary (elliptic or stable case). A matrix with

$$
\lambda_{1}=\lambda_{2}= \pm 1
$$

(singular case, $\omega=0)$ is not generic [Eq. (7b) can be violated by an arbitrarily small deformation of $M]$, so typically Eq. (7b) will hold only at some isolated critical value of $\varepsilon$.

If multipliers are hyperbolic, the central orbit is unstable and the neighboring trajectories diverge. Otherwise, if multipliers are elliptic, this orbit is (linearly) stable: the neighbors may coil around the central orbit for long times, depending on the nature of the nonlinear terms.

A fixed point of (6) satisfies the equation

$$
P_{\varepsilon}(x)-x=\left(M_{\varepsilon}-I\right) x+\cdots=0 .
$$

The solution $x_{\varepsilon}=0$ of this equation is unique if and only if

$$
\operatorname{det}\left(M_{\varepsilon}-I\right)=\prod_{j=1}^{2 N-2}\left(\lambda_{j}-1\right) \neq 0 .
$$

If the above determinant is nonzero at $\varepsilon_{0}$, then the PO persists (can be uniquely continued in $\varepsilon$ ) in a domain surrounding $\varepsilon_{0}$. Such a PO is called elementary ([16], Chap. VIII A1). Therefore Eq. (9a) is the analog of the Hessian condition (3b).
If $M_{\varepsilon}-I$ itself is nonsingular, singularity can still occur for $M_{\varepsilon}^{k}$ (repetitions of the Poincaré map). The $k$ th repetition of the periodic orbit is elementary (isolated and continuable) unless

$$
\begin{aligned}
& \operatorname{det}\left(M_{\varepsilon}^{k}-I\right)=0 \quad \text { for } \quad k=2,3, \ldots \\
& \lambda_{1,2}=\exp ( \pm 2 \pi n / k) \quad \text { for } n<k
\end{aligned}
$$

Conversely, at those critical values of $\varepsilon$ such that Eq. (9b) holds, a period- $k$ orbit might be created or destroyed at the central orbit.

The fundamental correspondence between the Hessian matrix (effectively - the matrix of the linearized equations near the origin) and the monodromy matrix is further explained in Secs. V A and V C.

\section{Reduction to two dimensions}

In a generic one-parameter family of matrices $M_{\varepsilon}^{k}$ Eq. (9a) can only be violated at an isolated critical value $\varepsilon_{\text {crit }}$ $\left[d \lambda\left(\varepsilon_{\text {crit }}\right) / d \varepsilon \neq 0\right.$, cf. Sec. II A 1], and Eq. (9b) does not hold at the same $\varepsilon_{\text {crit }}$. This assures that the bifurcation itself is generic and that no other period- $k$ bifurcation occurs for parameter values within some (finitely) small open neighborhood of $\varepsilon_{\text {crit }}[25]$.

In a generic theory those $\lambda_{j}(\varepsilon)$ in Eq. (9a) that belong to different pairs (7a) are independent functions of the parameter $\varepsilon$, and, therefore, only one pair $\lambda_{1,2}$ satisfies Eq. (9c) at a "time," i.e., at an isolated value $\varepsilon_{\text {crit }}$. The eigenvectors associated with this pair of eigenvalues define a plane in phase space in which the bifurcation manifests itself. In this way, the generic one-parameter problem reduces to dimension two [24,26]; with more parameters, more complex bifurcations can occur [27].

The study of each individual one-parameter bifurcation reduces to the study of an equivalent one-degree-of-freedom time-dependent ( $2 \pi$-periodic) system. A more complex phenomenon in which new periodic orbits are created at $\varepsilon_{\text {crit }}$ is structurally unstable: it can be decomposed into a sequence of generic two-dimensional (2D) phenomena (at $\left.\varepsilon_{\text {crit }}^{\prime}, \varepsilon_{\text {crit }}^{\prime \prime}, \ldots\right)$ by an arbitrarily small deformation of the problem (of $M_{\varepsilon}$ ).

\section{Correspondence of phase portraits of normal forms and Poincaré surfaces of sections}

The Kol'mogorov-Arnol'd-Moser (KAM) theorem and the theorems of Poincaré and Birkhoff assure us that around any stable PO of a nonintegrable system, a surface of section (SOS) will generally be horribly complicated, with highorder island chains, $X$ points, heteroclinic tangles, and structure within structure to all levels of resolution. How can the simple functions given in Table I describe bifurcations of periodic orbits in Hamiltonian systems? (i) Normal form theory replaces the exact Hamiltonian of the system by a new effective Hamiltonian, which possesses only integrable motion. (ii) The normal form correctly reproduces the largescale structures on the SOS; i.e., it creates an "interpolated", or "smoothed"' SOS. (iii) It does this effectively by averag- 
ing over high-order resonances in the system. The result preserves short-time local structure, but smooths the long-time structure.

The most important statement, which serves both as the premise and as the backbone of our work, is that despite the fact that the whole map is not reproduced by the normal form, the short-time PO's, their stability, and their bifurcations, are reproduced by the normal form. In particular the bifurcations of the fixed points of the map and of the corresponding PO's are completely equivalent to those of the stationary points of the normal form.

\section{Fixed versus $\varepsilon$-dependent coordinates}

In real life the problem usually needs certain coordinate transformations to achieve the standard normal form representation, such as in Table I. The first step is similar for functions $f_{\varepsilon}(q, p)$ and for PO's. For $C_{k}$ bifurcations the origin of the coordinate system is translated to coincide with the central stationary point of $f_{\varepsilon}$ (central equilibrium) for all values of $\varepsilon$. In the case of $\mathrm{PO}$ we construct coordinates that are normal to and have their origin at the central $\mathrm{PO}$ for all $\varepsilon$ 's (Sec. IV A and Appendix B). In general this coordinate transformation is $\varepsilon$ dependent.

The $C_{1}$ case is exceptional: on "one side" of the bifurcation there are no stationary points (PO's), and on "the other side" neither can be regarded as the "central" stationary point $(\mathrm{PO})$, so we cannot use their location to define the coordinate system. Instead, a fixed, $\varepsilon$-independent coordinate system is defined having its origin at the single degenerate stationary point (PO) that exists at $\varepsilon_{\text {crit }}$.

In the case of periodic orbits another $\varepsilon$ dependence comes into the coordinate system on a later step (Sec. IV A): when considering the motion along the PO we change to angleaction variables $(\theta, J)$ in which one period of the central orbit $T_{\varepsilon}$ maps into an interval $\Delta \theta=2 \pi$. In other words we use an $\varepsilon$-dependent effective time $(\theta)$ scale. This happens for all $C_{k}, k \geqslant 2$. In the $C_{1}$ case we simply lock our $\theta$ scale to the period $T_{\varepsilon_{\text {crit }}}$.

\section{Resonances and symmetries}

In Sec. II A symmetry was imposed as an a priori property and all possible canonical symmetries in the plane were considered $[22,23,28]$. In contrast, bifurcation theory of periodic orbits $[9,16]$ does not begin with any postulated symmetries. In fact we deal with generic periodic solutions, which have no special symmetry properties. Instead, symmetries emerge from the normal form procedure.

How this happens for periodic orbits will become explicitly clear after the normal form procedure is presented in Sec. VII [see Eq. (66)]. Here we give a simple example of a $k=3$ resonance of a periodic orbit ([29], Appendix 7). To study bifurcations of a $\mathrm{PO}$ we consider small oscillations about this PO [in the normal plane $\left.\left(q_{1}, p_{1}\right)\right]$ driven by the motion along the PO. Therefore, let us consider a onedimensional oscillator with phase space $\left(q_{1}, p_{1}\right)$ driven by a periodically oscillating force $F\left(q_{1}, p_{1} ; \theta\right)$, with $\theta$ the effective time. It is helpful to write $\left(q_{2}, p_{2}\right)=(\sin \theta, \cos \theta)$, and to think of $\left(q_{2}, p_{2}\right)$ as coordinates that are associated with motion along the central PO. Then the effective Hamilton function of this nonautonomous problem is

$$
\begin{gathered}
H\left(q_{1}, p_{1} ; \theta\right)=\omega \frac{1}{2}\left(q_{1}^{2}+p_{1}^{2}\right)+F, \\
F=F\left(q_{1}, p_{1} ; \theta\right)=F\left(q_{1}, p_{1} ; q_{2}, p_{2}\right),
\end{gathered}
$$

or in complex coordinates $z=q+i p$ [30],

$$
\begin{aligned}
H & =i \omega z_{1} z_{1}^{*}+2 i F\left(z_{1}, z_{1}^{*}, z_{2}, z_{2}^{*}\right) \\
& =i \omega z_{1} z_{1}^{*}+2 i F\left(z_{1}, z_{1}^{*}, e^{ \pm i \theta}\right) .
\end{aligned}
$$

The force $F$ is nonlinear, i.e., it depends on $\left(q_{1}, p_{1}\right)$ and the degree of $F$ in $\left(q_{1}, p_{1}\right)$ is greater than 2 . We consider sufficiently small oscillations in $\left(q_{1}, p_{1}\right)$ and represent $F$ as a power series in $z_{1}, z_{1}^{*}, z_{2}, z_{2}^{*}$. The normal form reduction eliminates from this series as many terms as possible. It retains only "resonant" or "secular" terms in $F$, those whose time derivatives are small:

$$
\frac{d F_{\text {res }}}{d \theta} \approx \frac{\partial F_{\text {res }}}{\partial \theta}+\omega \frac{1}{2}\left\{q_{1}^{2}+p_{1}^{2}, F_{\text {res }}\right\}=0 .
$$

If $\omega$ happens to be $1 / 3$ [the frequency of the driver is three times the natural frequency in $\left.\left(q_{1}, p_{1}\right)\right]$ the normal form retains the 1:3 resonance terms:

$$
F_{1: 3}=i\left[z_{1}^{3} z_{2}^{*}+\left(z_{1}^{*}\right)^{3} z_{2}\right]+\cdots .
$$

In action-angle variables, such that $z_{1}=\sqrt{2 I_{1}} e^{i \phi_{1}}$,

$$
F_{1: 3}=4 I_{1}^{3 / 2} \cos \left(3 \phi_{1}-\theta\right)+\cdots .
$$

Consider now (12a) in the plane $\left(q_{1}, p_{1}\right)$ (the plane of the Poincaré surface of section) at $q_{2}=0\left(\theta=0, p_{2}=1\right)$,

$$
\left.F_{1: 3}\right|_{q_{2}=0}=\left(p_{1}^{3}+3 p_{1} q_{1}^{2}\right) p_{2}+\cdots .
$$

This term is invariant with respect to $C_{3}$ rotation in the $\left(q_{1}, p_{1}\right)$ plane. Furthermore, the same $C_{3}$ symmetry persists at all $\theta$ if we use a coordinate system that rotates with $\theta$, such as $\varphi=\phi_{1}-\theta / 3$. [This coordinate system rotates in the $\left(q_{1}, p_{1}\right)$ plane as we move along the central PO, cf. Appen$\operatorname{dix}$ A 3.]

Normalization thus creates $C_{3}$ symmetry out of a general Hamiltonian. However, we must be cautious since we have not considered convergence of the normalization process. While $C_{3}$ is the exact symmetry of a truncated normal form, the only safe statement about the flow of the initial system is that it is approximately $C_{3}$ symmetric; i.e., it is symmetric in the same average sense as discussed in Sec. II B 3 ([29], Appendix 7). We return to this in Sec. IX B 1.

\section{Classification of bifurcations}

Now let us reexamine and reinterpret Table I in terms of bifurcations of periodic orbits. We only need to say that a saddle (X point) in the function plot corresponds to an unstable $\mathrm{PO}$ and a maximum or minimum (O point) corresponds to a stable PO.

a. $C_{1}$ bifurcation, saddle node, or extremal orbit. At the critical point $\varepsilon_{\text {crit }}$ condition (9a) is violated, Eq. (8) has more than one solution, and the stability of the orbit is undefined. The orbit is extremal ([16], Chap. VIII A 2), which means that, like the stationary points of (2a) it cannot be continued 
near $\varepsilon_{\text {crit }}$ [the two solutions of Eq. (8) exist either for $\varepsilon \geqslant \varepsilon_{\text {crit }}$ or else for $\varepsilon \leqslant \varepsilon_{\text {crit }}$. Two orbits get "created" or "annihilated" as a stable-unstable pair corresponding to the stationary points of the $C_{1}$ function in Table I.

b. $C_{2}$ bifurcation, period doubling, or transitional orbit. The "transitional" orbit ([16], Chap. VIII A 3) is so named because it changes stability while remaining on the scene. The surface of section looks like the plots of the $C_{2}$ function in Table I. The twin stationary points that (dis)appear at the origin are equivalent under the $C_{2}$ symmetry operation; the corresponding two fixed points of the Poincare map are equivalent as well, because they lie on the same period-2 orbit, and the period-1 Poincare map (6) maps them into each other. We may call such points connected.

c. Period-k or $C_{k}, k>2$, bifurcation. If there is a pair of multipliers $(9 \mathrm{c})$ the linear stability for the $k$ th repetition of the central orbit becomes undetermined, and it undergoes a period- $k$ bifurcation ([16], Chap. VIII A 4). In the "weakresonance" case (last row of Table I), the "island chain" pattern occurs: a stable-unstable pair of period- $k$ orbits, which wind $n$ times around the central orbit when passing $k$ times along it, branch from the central orbit. The latter is stable on either side of the bifurcation. In the case of strong resonance ( $C_{3}$ and upper line of $C_{4}$ in Table I) no new orbits are created at the center-the reason that it is called "touch and go" [6].

\section{Vocabulary}

Readers who are not familiar with the language of Hamiltonian bifurcation theory should note certain words that have different meanings in other areas of physics.

Given a collection of differential equations $\dot{x}_{i}=f_{i}\left(x_{1} \cdots x_{n}\right)$, the set of functions $f_{i}\left(x_{1} \cdots x_{n}\right)$ is said to define a vector field. That vector field is Hamiltonian if the differential equations can be derived from a Hamilton function: $n$ is even, the set $x_{i}$ is divided into $n / 2$ pairs $\left(q_{i}, p_{i}\right)$, and there exists a function $H(p, q)$ such that $\dot{q}_{i}=\partial H / \partial p_{i}, \dot{p}_{i}=-\partial H / \partial q_{i}$. A Hamiltonian matrix is a matrix that represents linear Hamiltonian equations of motion. (A Hamiltonian matrix is not Hermitian.) For more precise definitions and more details, see [16,29].

\section{SKETCH OF THE METHOD}

Arnol'd prepares us well by remarking that metamorphoses of phase portraits of (Hamiltonian) vector fields near a singular point and those of a family of trajectories in the neighborhood of a periodic orbit constitute the two "most complex problems of bifurcation theory" ([17], Sec. 6.34). The plot of our method is as follows. For a given periodic trajectory of a system with $N$ degrees of freedom, parameters $\varepsilon$, and Hamilton function $H_{\varepsilon}(p, q)$ we (1) use angle-action variables $(\theta, J)$ and normal variations $\left(q_{i}, p_{i}\right)$, $i=1, \ldots, N-1$, to describe the motion along and normal to the trajectory (Sec. IV A); (2) solve $\mathcal{H}_{\varepsilon}(\theta, J, q, p)=E$ and construct a reduced Hamilton function $\mathcal{J}_{E, \varepsilon}(q, p ; \theta)$, which is $2 \pi$ periodic in "time" $\theta$ and is parametrized by energy $E$ and parameter(s) $\varepsilon$ (Secs. IV B and IV C 2); (3) derive linearized equations for $\mathcal{J}_{E, \varepsilon}$ and obtain their fundamental matrix solution $X_{E, \varepsilon}(\theta)$ (Sec. V A 2); (4) study the monodromy matrix $M=X_{E, \varepsilon}(2 \pi)$ and identify critical value(s) $\varepsilon_{n: k}$ and corresponding resonant $2 \times 2$ subspace(s) $(\widetilde{q}, \widetilde{p})$ where $M_{\varepsilon}$ either has eigenvalues $\pm i 2 \pi n / k(k>2)$, or cannot be diagonalized $(k \leqslant 2, n=1)$; (5) use a Floquet transformation to obtain a time-independent linear part with matrix $\Omega=\ln M_{E, \varepsilon}$ (Secs. V B 3 and V D); (6) near each critical value, construct normal forms $\mathcal{J}_{n: k}(q, p)$, which are $C_{k}$ symmetric functions on the plane containing only $n: k$ resonance terms (Secs. VII and VIII); (7) consider bifurcations of the stationary points of these normal forms $\mathcal{J}_{n: k}(q, p)$ in the neighborhood of the origin $q=p=0$ corresponding to bifurcations of periodic orbits near and including the given (central) orbit (Secs. VII B and VIII B).

In step (6), to obtain the normal form of the periodic Hamilton function $\mathcal{J}_{E, \varepsilon}(q, p ; \theta)$, we transform nonautonomous equations of motion into autonomous but nonHamiltonian equations and then transform the latter using a normal-form algorithm for ordinary differential equations (vector fields) [31]. The normal form of $\mathcal{J}_{E, \varepsilon}$ is obtained from the normal form of the differential equations. (Connection to a more familiar theory for Hamilton functions is summarized in Appendix E 1.)

The general formulation of our approach is clearly analytical but the realization is in most cases numerical. As the theory is developed in the following sections, each theoretical step is followed by numerical implementation for our example, the perpendicular orbit of DKP.

In this paper we focus on the generic dynamical aspects of the problem, such as the organization phenomenon, and on the instrumental role of normal form theory. We note that our example (the DKP) has a number of a priori symmetries [32] besides those created by the normal form near the resonances. This gives us an opportunity to distinguish between generic and particular symmetry-related aspects of the theory.

\section{EQUATIONS IN NORMAL VARIATIONS NEAR PERIODIC ORBIT}

To study bifurcations of a periodic orbit we study the change of nearby orbits (the change of normal variations about the periodic orbit) caused by the change of parameter(s) $\varepsilon$. These variations can be found for any phase curve of a system of $2 N$ nonlinear differential equations. In the vicinity of the phase curve, one of the coordinates, let us call it $\theta$, may be chosen along the curve, so that other $2 N-1$ coordinates span the space of normal variations ( [17], Secs. $5.26 \mathrm{G}$ and $6.34 \mathrm{~A})$. Furthermore, $\theta(t)$ must be monotonic: $d \theta(t) / d t>0$ for all $t$, so that $t(\theta)$ is defined everywhere, and we can rewrite our initial system (near the phase curve) as a system of $2 N-1$ equations with coefficients depending on $\theta$, the new independent "time" coordinate. If the phase curve is a $T_{\varepsilon}$-periodic orbit (in general the period depends on the parameter $\varepsilon$ ) the coefficients are (for a properly chosen $t \rightarrow \theta) 2 \pi$-periodic functions of $\theta$.

\section{A. Normal variations}

We first introduce new coordinates in configuration space $(\lambda, \sigma)=\left(\lambda, \sigma_{1}, \ldots, \sigma_{N-1}\right)$ such that $\delta \sigma$ 's are variations 
normal to the configuration-space image of the trajectory. For $N=2$ this was explained in detail in Ref. [7], Appendix D1, and we give a shorter description in our Appendix B.

In the new canonical coordinates $\left(\lambda, p_{\lambda}, \sigma, p_{\sigma}\right)$, restriction of our equations of motion to the central trajectory is obviously given by $\sigma=p_{\sigma}=0$, resulting in a one-degree-offreedom problem with Hamiltonian $H_{\varepsilon}\left(\lambda, p_{\lambda}\right)$. Thereby $\delta \sigma$ and $\delta p_{\sigma}$ define $2 N-2$ variations normal to the central trajectory in the phase space of our dynamical system. On the other hand, neither $\delta \lambda$ nor $\delta p_{\lambda}$ are normal variations, nor can they in general be used as a new independent "time" variable. It is the action-angle variables

$$
\begin{gathered}
J=\frac{1}{2 \pi} \oint p_{\lambda} d \lambda=\frac{1}{2 \pi} \int_{0}^{T_{\varepsilon}} p_{\lambda} \dot{\lambda} d t \\
\theta(t)=\frac{2 \pi}{T_{\varepsilon}} t=\left(\frac{d \mathcal{E}_{\varepsilon}}{d J}\right) t, \quad H_{\varepsilon}\left(p_{\lambda}, \lambda\right)=\mathcal{E}_{\varepsilon}(J),
\end{gathered}
$$

that suit the purpose ([33], Chap. 7, Sec. 4.1, Proposition 1). Indeed, $\delta J$, variation of the action of the central trajectory, is clearly the last needed normal variation, and $\theta$ is the new "time" variable. This important dynamical concept completes our construction of the space of normal variations for a periodic trajectory of a Hamiltonian system.

\section{B. Isoenergetic reduction}

For time-independent dynamical systems we account for energy conservation

$$
H_{\varepsilon}\left(J, p_{\sigma}, \sigma ; \theta\right)=E=\mathrm{const},
$$

i.e., we consider normal variations of the orbit restricted to the set of constant energy $E$. Any variation $\delta J$ and hence $\delta \mathcal{E}_{\varepsilon}(J)=2 \pi \delta J / T_{\varepsilon}$, the energy variation of the periodic orbit, should be "compensated" by variation of the energy of the motion in the normal space $\left(p_{\sigma}, \sigma\right)$. In other words, of the $2 N-1$ normal variations $\left(\delta J, \delta p_{\sigma}, \delta \sigma\right)$ of a conservative dynamical system only $2 N-2$ are independent. These latter are called isoenergetic normal variations. It follows that at any given $\theta$ Eq. (14a) defines a $(2 N-2)$-dimensional space of isoenergetic normal variations, embedded in $\left(J, p_{\sigma}, \sigma\right)$. In that $(2 N-2)$-dimensional space the flow is Hamiltonian and the reduced Hamilton function $\mathcal{J}_{\varepsilon, E}\left(p_{\sigma}, \sigma, \theta\right)$ is obtained by solving Eq. (14a) for $J$ as a function of $\left(p_{\sigma}, \sigma, \theta\right)$ at fixed $\varepsilon$ and $E$ :

$$
\mathcal{J}_{\varepsilon, E}\left(p_{\sigma}, \sigma, \theta\right)=-J .
$$

This reduced Hamilton function is $2 \pi$ periodic in $\theta$ (in "time") and it contains energy $E$ as well as $\varepsilon$ as parameters. The solution of (14a) to obtain (14b) is possible locally by the implicit function theorem because [34]

$$
\frac{\partial H_{\varepsilon}\left(J, p_{\sigma}, \sigma ; \theta\right)}{\partial J} \sim \frac{\partial \mathcal{E}_{\varepsilon}}{\partial J}=\frac{2 \pi}{T_{\varepsilon}} \neq 0 .
$$

Following Arnol'd we call this procedure isoenergetic reduction ([29], Sec. 6.45B and Appendix 7C). This time- dependent Hamiltonian (14b) describes the evolution on the single energy shell (energy level set) in the neighborhood of this central orbit.

The concept of isoenergetic normal variations is in a sense a generalization of the Poincare surface of section method. In effect we are working with a continuous set of surfaces of section $S(\theta)$ taken at different "times" $\theta$, along the central orbit. Each $S(\theta)$ is a phase portrait (a constant level set), a slice of the extended phase space of Hamilton function $J_{\varepsilon, E}$ and that Hamilton function describes the continuous $\theta$ evolution of these sections. The "conventional" Poincaré surface of section $\tilde{S}$ is defined as a hypersurface (usually a plane) in the (initial) phase space $(q, p)$ transverse to the central trajectory. The latter crosses $\widetilde{S}$ at some $\left(\theta_{0}, q_{0}, p_{0}\right)$ and there are diffeomorphic open neighborhoods of $\left(q_{0}, p_{0}\right)$ on $\widetilde{S}$ and on $S\left(\theta_{0}\right)$.

\section{Perpendicular orbit in the diamagnetic Kepler problem}

To illustrate this method we consider a single electron (such as in the hydrogen atom) that moves in Coulomb and magnetic fields with orbital angular momentum $L_{z}=0$. As shown in Appendix $\mathrm{C}$ the Hamilton function for this problem,

$$
H_{\varepsilon}=2=\frac{p_{u^{\prime}}^{2}+p_{v^{\prime}}^{2}}{2}+4\left(u^{\prime 2}+v^{\prime 2}\right)\left[\frac{\left(u^{\prime 2}-v^{\prime 2}\right)^{2}}{2}-\varepsilon\right],
$$

is nonsingular in the so-called semiparabolic coordinates (C2). We study one particular periodic trajectory, which is normal to the field, the so-called perpendicular orbit. On every major step our general outline will be followed by the concrete application to this orbit.

\section{Perpendicular trajectory}

Equations of motion for this particular solution follow from Hamilton function (15) restricted to $v^{\prime} \equiv 0$ :

$$
\mathcal{H}_{\varepsilon}\left(p_{\lambda}, \lambda\right)=\frac{1}{2} p_{\lambda}^{2}-4 \varepsilon \lambda^{2}+2 \lambda^{6}=2, \quad \lambda=u^{\prime} .
$$

No transformation of configuration coordinates is required since in (16) the role of translational and normal coordinates $(\lambda, \sigma)$ is played by $\left(u^{\prime}, v^{\prime}\right)$. The solution $\Gamma_{\varepsilon}(\theta)=\left[p_{\lambda}(\theta, \varepsilon), \lambda(\theta, \varepsilon)\right]$ for Hamilton equations defined by (16) with initial condition $t=\lambda=0$ is shown in Fig. 3; it can be represented as a (vector valued) Fourier series

$$
\begin{gathered}
\Gamma_{\varepsilon}(\theta)=\left[\begin{array}{l}
Q_{\varepsilon}(\theta) \\
P_{\varepsilon}(\theta)
\end{array}\right]=\sum_{k=1,3,5, \ldots}\left[\begin{array}{l}
Q_{\varepsilon}^{k} \sin (k \theta) \\
P_{\varepsilon}^{k} \cos (k \theta)
\end{array}\right], \\
\theta=\frac{2 \pi t}{T_{\varepsilon}}, \quad P_{\varepsilon}^{k}=\frac{2 \pi k}{T_{\varepsilon}} Q_{\varepsilon}^{k} .
\end{gathered}
$$

Due to the simple kinetic term in (16) $\dot{Q}_{\varepsilon}=P_{\varepsilon}$, and hence (17b). The dependence of the Fourier amplitude $Q_{\varepsilon}^{1}$ in Eq. (17), period $T_{\varepsilon}$, and action $J_{\varepsilon}$ in Eq. (13a) on the parameter $\varepsilon$ is illustrated in Fig. 4. Notably, the "harmonic" term $Q^{1}$ accounts for up to $90 \%$ of $\lambda(\theta)$, and its contribution natu- 


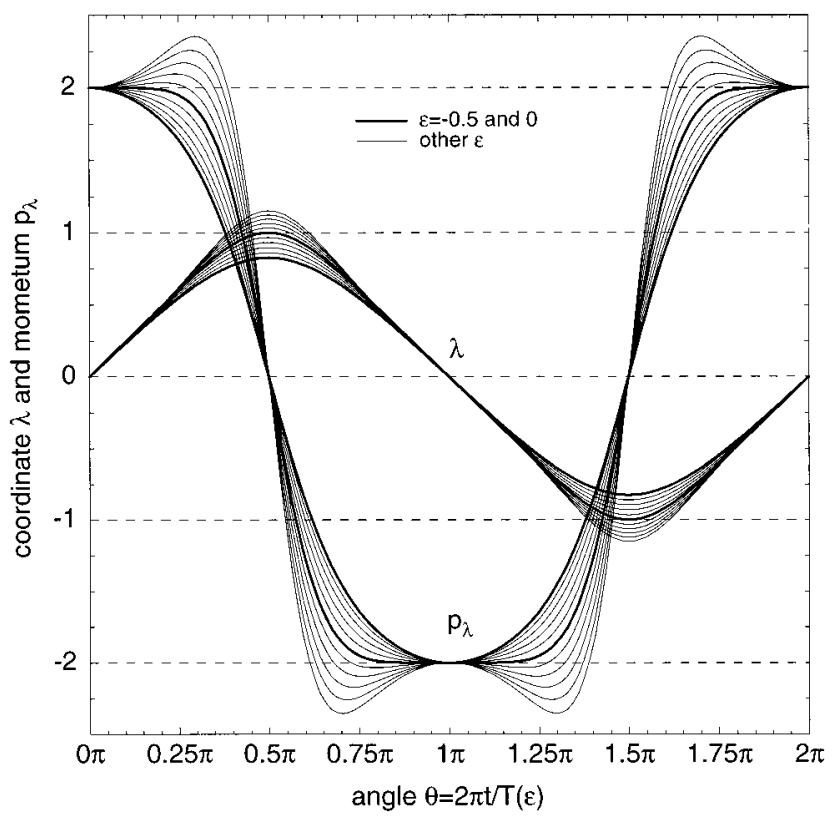

FIG. 3. Perpendicular periodic orbit in the diamagnetic Kepler problem: solution $\lambda(\theta), p_{\lambda}(\theta)$ at different values of scaled energy $\varepsilon=-0.5, \ldots, 0, \ldots, 0.5$.
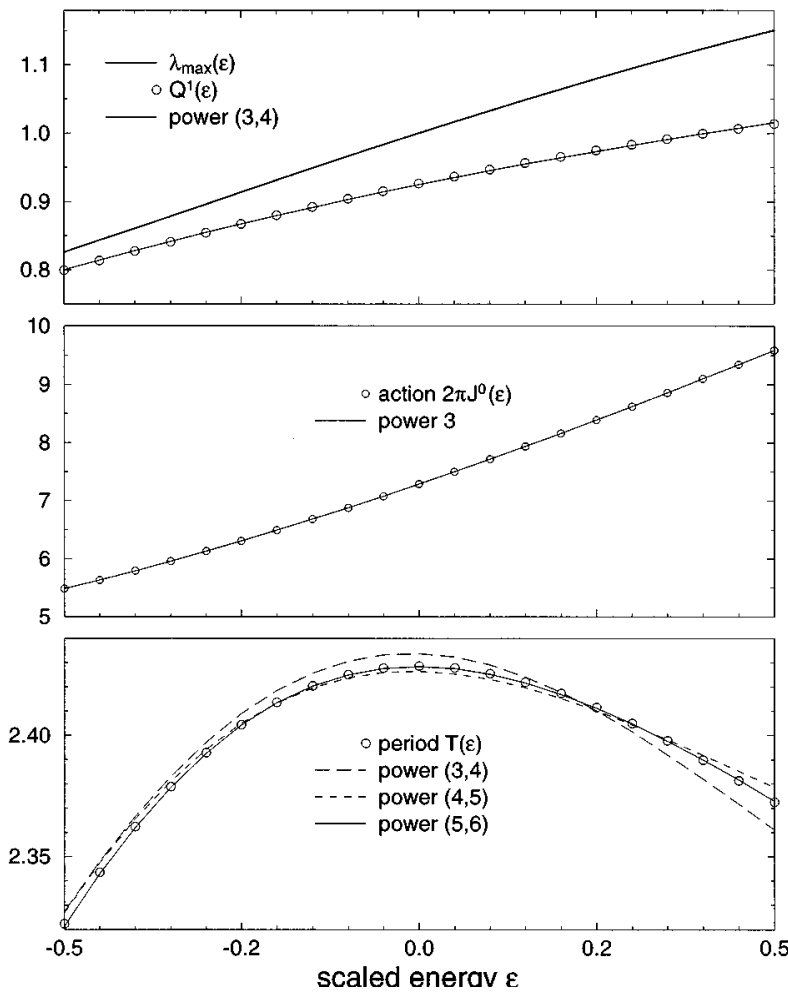

FIG. 4. Amplitude $\lambda_{\max }$, first Fourier series coefficient $Q^{1}$ (top plot), action $J^{0}$ (middle plot), and period $T$ of perpendicular orbit as functions of scaled energy $\varepsilon . J^{0}(\varepsilon) \equiv J_{\varepsilon}^{0}$ in Eq. (21) is approximated by a power 3 polynomial. $Q^{1}(\varepsilon)$ is $Q_{\varepsilon}^{k=1}$ in Eq. (17) or $Q_{\varepsilon}^{k=1}\left(J^{0}\right)$ in Eq. (19b). This $Q^{1}(\varepsilon)$ is obtained from an approximating polynomial of power $(3,4)$ in $(\varepsilon, J) . T_{\varepsilon}$ is obtained from different approximating polynomials for virtual energy $\mathcal{E}(\varepsilon, J)$ as $(\partial \mathcal{E} / \partial J)^{-1}$ at $J^{0}$. rally decreases with growing $\varepsilon$. In general we can qualitatively approximate $Q_{\varepsilon}^{k}$ and $J_{\varepsilon}$ by linear, and $T_{\varepsilon}$ by quadratic functions of $\varepsilon$.

\section{Equations in normal variations}

For the (regularized) diamagnetic Kepler problem, $E$ in Eq. (14a) has a fixed value, equal to 2 , while $\varepsilon$, or " scaled energy," is the only parameter. Nevertheless, the method of normal variations begins by introducing an additional parameter, the virtual energy $\mathcal{E}$, and solving the Hamiltonian problem (16) at different values of this virtual energy:

$$
\mathcal{H}_{\varepsilon}\left(p_{\lambda}, \lambda\right)=\frac{1}{2} p_{\lambda}^{2}-4 \varepsilon \lambda^{2}+2 \lambda^{6}=\mathcal{E}=2+\delta \mathcal{E} .
$$

( $\delta \mathcal{E}$ is the virtual energy going into transverse oscillations when the trajectory deviates from $\sigma=p_{\sigma}=0$.) With the introduction of this virtual energy, the transformation to action-angle representation proceeds as a textbook onedimensional problem. For each scaled energy $\varepsilon$, the trajectory is computed at several values of virtual energy $\mathcal{E}$, the action $J=J_{\varepsilon}(\mathcal{E})$ is evaluated as a function of virtual energy $\mathcal{E}$, and finally the canonical transformation $\left(\lambda, p_{\lambda}\right) \leftrightarrow(\theta, J)$ is expressed as Taylor-Fourier series,

$$
\begin{gathered}
{\left[\begin{array}{c}
Q_{\varepsilon}(J, \theta) \\
P_{\varepsilon}(J, \theta)
\end{array}\right]=\sum_{\text {odd } k}\left[\begin{array}{c}
Q_{\varepsilon}^{k}(J) \sin (k \theta) \\
\frac{2 \pi k}{T_{\varepsilon}(J)} Q_{\varepsilon}^{k}(J) \cos (k \theta)
\end{array}\right],} \\
Q_{\varepsilon}^{k}(J)=\sum_{i=0} \sum_{j=1} \frac{Q_{i j}^{k}}{(i+j) !} \varepsilon^{i} J^{j}, \\
\mathcal{H}_{\varepsilon}(J)=\sum_{i=0} \sum_{j=1} \frac{\mathcal{E}_{i j}}{(i+j) !} \varepsilon^{i} J^{j},
\end{gathered}
$$

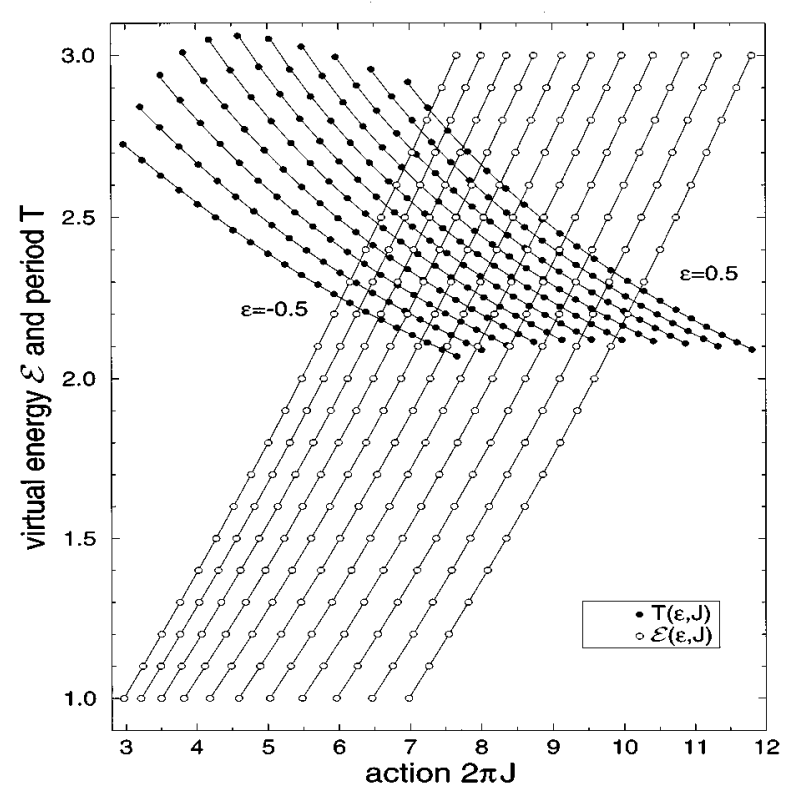

FIG. 5. Variational study of perpendicular trajectory. Circles show exact values of virtual energy $\mathcal{E}$ and period $T$. $\mathcal{E}$ is approximated by a power 6 polynomial in scaled energy and action $(\varepsilon, J)$ with no $J$-independent terms; $T(\varepsilon, J)$ is obtained from the approximation for $\mathcal{E}$ and Eq. (19d). 
TABLE II. Leading terms in the power series approximation for virtual energy $\mathcal{E}(19 \mathrm{c})$, and Fourier amplitudes $Q^{k}(19 \mathrm{~b})$ in the range $-0.5 \leqslant \varepsilon \leqslant 0$ (11 data points) and $-1 \leqslant \delta \mathcal{E} \leqslant 1$ (11 points).

\begin{tabular}{lcccccc}
\hline \hline$i j$ & $\mathcal{E}_{i, j}$ & $10 Q_{i, j}^{1}$ & $10^{2} Q_{i, j}^{3}$ & $10^{3} Q_{i, j}^{5}$ & $10^{3} Q_{i, j}^{7}$ & $10^{4} Q_{i, j}^{9}$ \\
\hline 01 & 0.3520 & 4.656 & -0.1936 & -0.0015 & 0.0071 & -0.0022 \\
02 & 4.427 & -28.16 & 0.2880 & 0.3206 & -0.1319 & 0.0347 \\
11 & -9.724 & 5.131 & -2.452 & 0.1129 & 0.0451 & -0.0180 \\
03 & -11.38 & 159.6 & 2.729 & -3.289 & 1.158 & -0.2925 \\
12 & 60.06 & -59.87 & 16.80 & 2.822 & -1.620 & 0.4397 \\
21 & -20.26 & -2.081 & -13.70 & 1.681 & -0.0979 & -0.0256 \\
04 & 36.92 & -757.9 & -31.25 & 21.54 & -7.113 & 1.761 \\
13 & -351.5 & 383.8 & -31.89 & -48.84 & 18.73 & -4.538 \\
22 & 283.0 & -48.84 & 155.0 & -2.923 & -4.884 & 1.758 \\
31 & -9.369 & -43.64 & -28.65 & 7.635 & -1.689 & 0.2953 \\
05 & -99.89 & 2858.0 & 179.5 & -100.3 & 31.93 & -7.807 \\
14 & 1530 & -1247.0 & -380.9 & 397.7 & -128.7 & 28.91 \\
23 & -2069 & 805.0 & -745.5 & -115.6 & 69.80 & -19.11 \\
32 & 301.7 & 386.4 & 366.6 & -50.96 & 2.625 & 1.381 \\
41 & 60.21 & -46.43 & 8.229 & 11.29 & -5.025 & 1.423 \\
15 & -4419 & -1233.0 & 3965 & -2018. & 575.6 & -120.2 \\
24 & 7655 & -3586.0 & 464.8 & 828.9 & -306.7 & 70.13 \\
33 & -2235 & -416.4 & -1441 & -100.9 & 103.8 & -33.33 \\
42 & -469.9 & 281.4 & -129.1 & -69.13 & 30.68 & -7.622 \\
51 & -14.63 & 37.80 & 14.62 & -4.680 & 0.3694 & 0.3100 \\
\hline \hline
\end{tabular}

$$
T_{\varepsilon}(J)=2 \pi\left[\frac{\partial \mathcal{H}_{\varepsilon}(J)}{\partial J}\right]^{-1}
$$

The virtual energy $\mathcal{E}$ and Fourier amplitudes $Q^{k}$ vanish when $J \rightarrow 0$ and hence have no $J$-independent terms. The dependence of virtual energy (19c) and period (19d) on $J$ and $\varepsilon$ is shown in Fig. 5; typically, to obtain a 4-5 digit accuracy in $T_{\varepsilon}$ a power $(5,6)$ approximation polynomial in $(\varepsilon, J)$ is required for $\mathcal{E}(\varepsilon, J)$. Table II gives the numerical values of coefficients in Eqs. (19b) and (19c).

To arrive at the time-dependent problem (14a) we transform the total Hamiltonian $H_{\varepsilon}\left(p_{u^{\prime}}, u^{\prime}, p_{v^{\prime}}, v^{\prime}\right)$

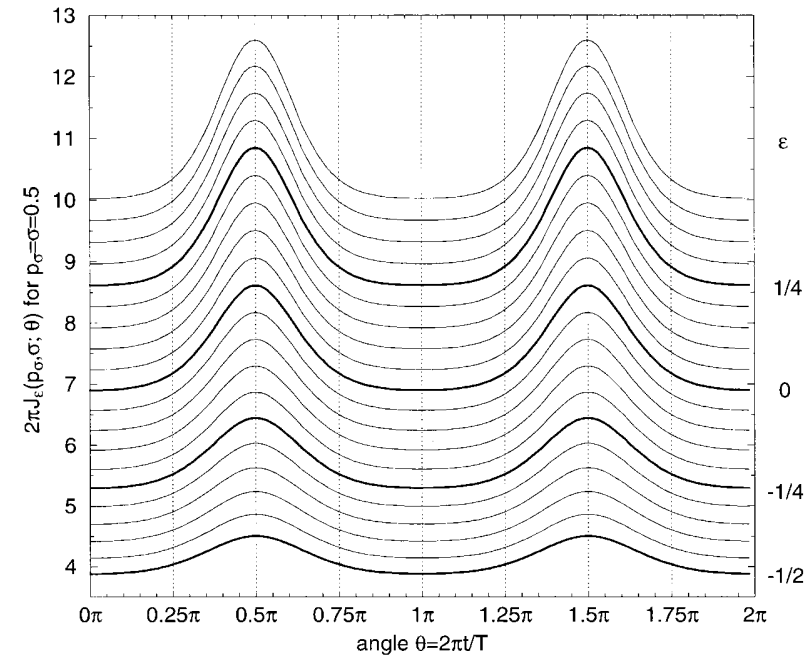

FIG. 6. Reduced Hamiltonian in normal variations near perpendicular trajectory (21) as a function of angle variable $\theta$, for $\sigma=p_{\sigma}=0.5$ at different values of parameter $\varepsilon$. $\equiv H_{\varepsilon}\left(p_{\lambda}, \lambda, p_{\sigma}, \sigma\right) \quad$ in $\quad$ Eq. (15) by substituting $\left[\lambda, p_{\lambda}\right] \rightarrow\left[Q_{\varepsilon}(J, \theta), P_{\varepsilon}(J, \theta)\right]$ and obtain

$$
\begin{aligned}
H_{\varepsilon}\left(J, p_{\sigma}, \sigma ; \theta\right)= & \mathcal{H}_{\varepsilon}(J)+\left[\frac{p_{\sigma}^{2}}{2}-4 \varepsilon \sigma^{2}+2 \sigma^{6}\right] \\
& -2 \sigma^{2} Q_{\varepsilon}(J, \theta)^{2}\left[Q_{\varepsilon}(J, \theta)^{2}+\sigma^{2}\right] \\
= & 2 .
\end{aligned}
$$

For the transverse motion in $\left(p_{\sigma}, \sigma\right)$, the first term $\mathcal{H}_{\varepsilon}(J)$ is an additive "constant" that can be ignored. The next square bracket arises from those terms in Eq. (15) that contain only $p_{v^{\prime}}$ and $v^{\prime}$, and the last term describes periodic coupling of the motions along and normal to the trajectory.

Now the isoenergetic reduction to (14b): Eq. (20b) defines $J$ as an implicit function of $\left(p_{\sigma}, \sigma ; \theta\right)$, and we need to construct (minus) this function explicitly for $\left(p_{\sigma}, \sigma\right)$ sufficiently small [see discussion of Eq. (B2) in Appendix B]. This construction can be done analytically, but a numerical solution would do just as well: we solve Eq. (20a) at different values of $\left(\varepsilon, \sigma, p_{\sigma}\right)$ and $\theta$, tabulate the resulting data, and approximate them by a Taylor-Fourier series

$$
\begin{aligned}
\mathcal{J}_{\varepsilon, H=2}\left(p_{\sigma}, \sigma ; \theta\right)= & J_{\varepsilon}^{0}+\sum_{a=1}\left(J_{\varepsilon 0 a}^{0} p_{\sigma}^{2 a}+J_{\varepsilon a 0}^{0} \sigma^{2 a}\right) \frac{1}{a !} \\
& +\sum_{\substack{a=1 \\
b=0}} \frac{\sigma^{2 a} p_{\sigma}^{2 b}}{(a+b) !} \sum_{k=1} J_{\varepsilon a b}^{k} \cos (2 k \theta),
\end{aligned}
$$

with coefficients expanded in a power series in $\varepsilon$ : 


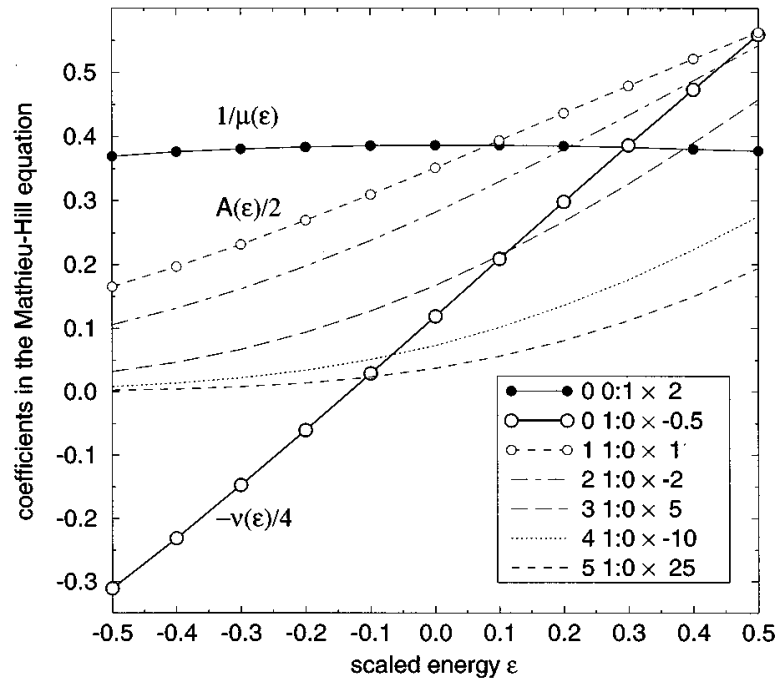

FIG. 7. Mathieu-Hill's equation for perpendicular trajectory: coefficients $J_{\varepsilon a b}^{k}$ with $a+b=1$ (denoted $\left.k a: b\right)$ in Eq. (21).

$$
J_{\varepsilon a b}^{k}=\sum_{i=0} \varepsilon^{i} J_{i a b}^{k} \frac{(a+b) !}{(a+b+i) !} .
$$

An example of such a solution is shown in Fig. 6. There are no terms linear in $\left(p_{\sigma}, \sigma\right)$ since the force vanishes for $\left(p_{\sigma}, \sigma\right)=0$ (on the central trajectory); more precisely, as seen from Eq. (20a), the potential energy is always $\propto \sigma^{2}$. The first few coefficients in (21) are shown in Fig. 7. As expected, at $\varepsilon \gg 0$ high frequencies become increasingly important, but nevertheless it is possible to obtain a good FourierTaylor series approximation in a sufficiently wide range of $\left(p_{\sigma}, \sigma\right)$. Principal terms in $(21)$ are $p_{\lambda}^{2}, \lambda^{2}, \lambda^{2} \cos (2 \theta)$, and $\lambda^{4}$ with coefficients given below:

\begin{tabular}{llrccc}
$k$ & $a b$ & \multicolumn{1}{c}{$J_{0 a b}^{k}$} & $J_{1 a b}^{k}$ & \multicolumn{1}{c}{$J_{2 a b}^{k}$} & \multicolumn{1}{c}{$J_{3 a b}^{k}$} \\
\hline 0 & 01 & 0.1932 & 0.0004 & -0.1613 & 0.3636 \\
0 & 10 & -0.2379 & -3.608 & -0.0171 & 6.267 \\
1 & 10 & 0.0352 & 0.0844 & 0.0249 & -0.2255 \\
0 & 20 & -0.8223 & -2.172 & 19.78 & 17.87
\end{tabular}

For a physical interpretation, let us write (21) in simpler notation:

$$
\begin{aligned}
\mathcal{J}_{\varepsilon, H=2}= & J^{0}(\varepsilon)+\frac{1}{2} p_{\sigma}^{2} / \mu(\varepsilon)+\frac{1}{2}[\nu(\varepsilon)+A(\varepsilon) \cos 2 \theta+\cdots] \sigma^{2} \\
& +B(\varepsilon) \sigma^{4}+\cdots
\end{aligned}
$$

For the transverse motion, $J^{0}(\varepsilon)$ is an additive constant and can be ignored. The effective mass $\mu(\varepsilon)=\left[2 J_{\varepsilon 01}^{0}\right]^{-1}$ is close to 2.5 and nearly independent of $\varepsilon$. The effective force constant $\nu(\varepsilon)=2 J_{\varepsilon 10}^{0}$ varies approximately linearly with $\varepsilon$, being positive (restoring) when $\varepsilon \ll-0.12$ and negative (unstable) otherwise [cf. quadratic terms of the initial Hamilton function (15)]. The quantity $A(\varepsilon)=2 J_{\varepsilon 10}^{2}$ represents the force constant for parametrically driven oscillations. Finally $B(\varepsilon)=J_{\varepsilon 20}^{0}$ is the force constant for the nonlinear (cubic) restoring force. It is negative, indicating that the confinement

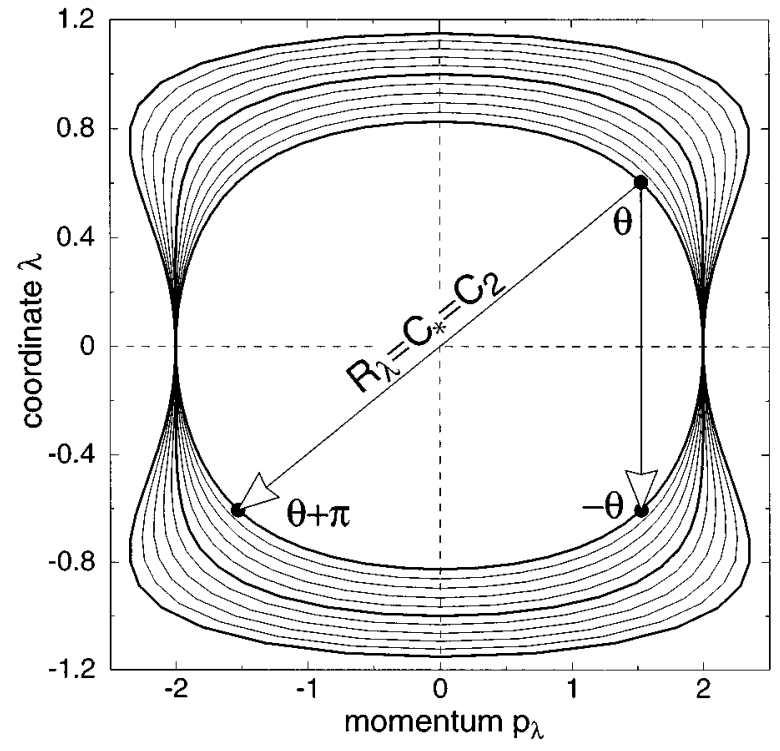

FIG. 8. Phase plot of perpendicular trajectory for $\varepsilon=-0.5 \cdots 0.5$. See Eqs. (24), (25), and (26).

of $\sigma$ is weaker than what would be obtained for a harmonic oscillator. The first three terms enter the linearized equations analyzed in Sec. V D below.

\section{Symmetry properties}

Symmetries of the reduced Hamilton function $\mathcal{J}_{\varepsilon}\left(p_{\sigma}, \sigma ; \theta\right)$ in Eqs. (21) and (22) affect the analytic structure of the normal form as well as the geometry of bifurcating orbits. $\mathcal{J}_{\varepsilon}\left(p_{\sigma}, \sigma ; \theta\right)$ is periodic in $\theta$ with period $\pi$, symmetric about $\theta=0$, and it contains only even powers of $\sigma$ and $p_{\sigma}$.

Let us see how symmetries of the original Hamiltonian (15) and (16) and of the periodic orbit (17) produce the symmetries of $\mathcal{J}$. Hamiltonian (15) is invariant under a number of linear canonical transformations of the phase space, but only two operations

$$
\begin{aligned}
& \mathrm{R}_{\lambda}:\left(\lambda, p_{\lambda}, \sigma, p_{\sigma}\right) \mapsto\left(-\lambda,-p_{\lambda}, \sigma, p_{\sigma}\right), \\
& \mathrm{R}_{\sigma}:\left(\lambda, p_{\lambda}, \sigma, p_{\sigma}\right) \mapsto\left(\lambda, p_{\lambda},-\sigma,-p_{\sigma}\right)
\end{aligned}
$$

that leave the perpendicular orbit invariant are relevant. Thus the invariance group of the perpendicular PO is

$$
\mathfrak{g}=\left\{I, \mathrm{R}_{\lambda}, \mathrm{R}_{\sigma}, C_{2}\right\} \sim D_{2}, \quad C_{2}=\mathrm{R}_{\lambda} \circ \mathrm{R}_{\sigma},
$$

a group of order four.

Due to our choice of initial conditions the origins of space inversion $\mathrm{R}_{\lambda}$ and time reversal $C_{*}:(p, \theta) \rightarrow(-p,-\theta)$ coincide, and $Q_{\varepsilon}$ and $P_{\varepsilon}$ in (17) are, respectively, odd and even functions of $\theta$. We also note (cf. Fig. 3) that since $\lambda=0$ for $\theta=0 \bmod \pi$ (at the origin) and $p_{\lambda}=0$ for $\theta=\pi / 2 \bmod \pi$ (at the turning points) the Fourier series contain no constant terms: $Q_{\varepsilon}^{0}(J)=P_{\varepsilon}^{0}(J)=0$ in Eq. (19). Moreover, since the perpendicular trajectory is degenerate [35] it is time-reversal invariant: for every $\theta$ there exists $\theta^{\prime}$ such that

$$
C_{*}\left(\begin{array}{l}
Q_{\varepsilon}(\theta) \\
P_{\varepsilon}(\theta)
\end{array}\right)=\left(\begin{array}{c}
Q_{\varepsilon}(-\theta) \\
-P_{\varepsilon}(-\theta)
\end{array}\right)=\left(\begin{array}{c}
Q_{\varepsilon}\left(\theta^{\prime}\right) \\
P_{\varepsilon}\left(\theta^{\prime}\right)
\end{array}\right) .
$$


( $\theta^{\prime} \neq \theta$ since $Q_{\varepsilon}$ is odd.) This and the idempotency of $C_{*}$ mean that if applied to $\left[Q_{\varepsilon}(\theta), P_{\varepsilon}(\theta)\right]$ the time-reversal operation reduces to (see Fig. 8)

$$
C_{*}: \theta \mapsto \theta^{\prime}=\theta+\pi, \quad p_{\lambda} \mapsto-p_{\lambda} .
$$

Equations (24) and (25) can be satisfied if only odd- $k$ Fourier harmonics (starting with $k=1$ ) are present in (17). Considering action (25) on the perpendicular orbit (17) we conclude that in fact

$$
C_{*}\left(\begin{array}{l}
Q_{\varepsilon}(\theta) \\
P_{\varepsilon}(\theta)
\end{array}\right)=C_{2}\left(\begin{array}{l}
Q_{\varepsilon}(\theta) \\
P_{\varepsilon}(\theta)
\end{array}\right)=\left(\begin{array}{c}
-Q_{\varepsilon}(\theta) \\
-P_{\varepsilon}(\theta)
\end{array}\right),
$$

i.e., the action of the time-reversal operation on the perpendicular orbit (17) is identical to that of spatial inversion $C_{2}$.

\section{FLOQUET-LYAPUNOV THEORY}

We return now to general theory, and consider a general $2 \pi$-periodic-in- $\theta$ Hamilton function as in Eq. (14b). Such a Hamilton function is the starting point of the local qualitative study of the motion near the central PO. Referring to the sketch of the method in Sec. III we now carry out steps 3, 4, and 5: derive linear equations for the transverse motion, study the monodromy matrix of these equations, and use a Floquet transformation to make the linear part time independent.

The first important result of the linear theory is the stability analysis of the central periodic trajectory. Furthermore, as described in Sec. IV B phase portraits of Hamiltonian (14b) lead to a continuous periodic family of Poincaré surfaces $S\left(\sigma, p_{\sigma} ; \theta\right)$. The linear theory compensates for changes occurring in $S\left(\sigma, p_{\sigma} ; \theta\right)$ by a linear symplectic $2 \pi$-periodic-in- $\theta$ transformation of the transverse space $\left(\sigma, p_{\sigma}\right)$. In other words we adjust our reference frame by making a linear $2 \pi$-periodic canonical stretch and rotation of coordinates $\left(\sigma, p_{\sigma}\right)$ while traveling along the orbit. By the Floquet-Lyapunov theorem the linear part can be made $e x$ actly time independent using this change of variables. This transformation will subsequently be applied to the nonlinear part of (14b).

\section{A. Mathieu-Hill equations}

\section{Linearization of reduced Hamiltonian}

For any given $\theta$ the point $p_{\sigma}=\sigma=0$ is an equilibrium: the periodic force vanishes at the central trajectory, $\left.\dot{p}_{\sigma}\right|_{0}=\left.\dot{\sigma}\right|_{0}=0$. (The central trajectory corresponds to the central stationary point of a function of two variables, such as $f_{\varepsilon}$ in Sec. II A 2.) Hence, linearization of the equations of motion defined by $\mathcal{J}_{\varepsilon, E}$ in Eq. (14b) gives a quadratic Hamilton function such as, for example,

$$
\begin{gathered}
\mathcal{J}_{\varepsilon, E}^{\text {in }}=J^{0}(\varepsilon, E)+\frac{1}{2} p_{\sigma}^{2} / \mu(\varepsilon, E) \\
+\frac{1}{2} \sigma^{2}[\nu(\varepsilon, E)+A(\varepsilon, E) \cos \theta+\cdots], \\
\dot{\sigma}=p_{\sigma} / \mu, \\
\dot{p}_{\sigma}=-\sigma(\nu+A \cos \theta+\cdots),
\end{gathered}
$$

$$
\ddot{\sigma}=-\sigma(\mu \nu+A \cos \theta+\cdots) .
$$

Without high-frequency terms denoted by ellipses these are Mathieu equations [36]; Hill's equations [37] can have any periodic force. The following discussion applies to a generalization of (27): any quadratic Hamilton function having $N^{\prime}$ degrees of freedom.

\section{Fundamental matrix solution and monodromy matrix}

Equations (27) describe (small) oscillations near the central orbit driven by a $2 \pi$-periodic force that is proportional to the displacement. The fundamental matrix solution $X(\theta)$ of system (27b) (of a linear nonautonomous Hamiltonian system with $N^{\prime}=N-1$ degrees of freedom) is a real symplectic matrix of dimension $2 N^{\prime}$ such that

$$
X(0)=I, \quad \operatorname{det} X(\theta)=1, \quad X(\theta) \in \operatorname{Sp}\left(2 N^{\prime}, r\right) .
$$

Then any other matrix solution $Y(\theta)$ of $(27 \mathrm{~b})$ is $([16]$, Theorem 3, Chap. II A)

$$
Y(\theta)=X(\theta) Y(0) .
$$

In particular, since Eqs. (27) are $2 \pi$ periodic $Y(\theta)$ $=X(\theta+2 \pi)$ must be a solution and

$$
X(\theta+2 \pi)=X(\theta) X(2 \pi) .
$$

This shows that in essence, even though solutions of Eqs. (27b) are not periodic, the group of diffeomorphisms defined by these equations needs to be studied only for $0<\theta \leqslant 2 \pi$. The monodromy matrix

$$
M=X(2 \pi) \in \operatorname{Sp}\left(2 N^{\prime}, r\right), \operatorname{det} M=1
$$

defines the transformation over the period $2 \pi$.

\section{B. Floquet-Lyapunov theorem}

\section{Logarithm of a symplectic matrix}

Like any symplectic matrix, the monodromy matrix (28d) has a logarithm ([16], Chap. II, Theorem 2, and Appendix). However, matrix $\ln M$ can be real only if multipliers $\lambda$ (eigenvalues of $M$ ) are such that $\operatorname{Re} \lambda>-1$. Appendix $\mathrm{D}$ gives the solution for case (7a). If all multipliers are of type (7a) [24] the logarithm is a blockdiagonal matrix with $2 \times 2$ blocks as in Eq. (D4) [38].

\section{Theorem}

The fundamental matrix solution (28a) is not periodic, however, by the Floquet-Lyapunov theorem (see [16], Chap. II E, [17], Sec. 5.26 A, and [37], Chap. 1.2) it can be represented as

$$
X(\theta)=B(\theta) M(\theta)=B(\theta) \exp (\Omega \theta), \quad M(2 \pi)=M,
$$

with $B(\theta)$ a $2 \pi$ periodic symplectic matrix, and $\Omega$ a $\theta$-independent Hamiltonian matrix [cf. Eq. (A1)]. The periodicity of $B(\theta)$ simply follows from (28c):

$$
B(\theta+2 \pi)=X(\theta+2 \pi) M(\theta+2 \pi)^{-1}=X(\theta) M(\theta)^{-1} .
$$


Now we define a new reference frame $y$, which moves according to $B(\theta)$ :

$$
x=B(\theta) y,
$$

and our equations of motion (27) reduce to a linear timeindependent system

$$
\dot{y}=\Omega y \text {. }
$$

The proof is short and instructive to follow. We slightly extend this proof by applying transformation (29c) to the full nonlinear system:

$$
\dot{x}=A(\theta) x+u(x, \theta)=\dot{B} y+B \dot{y} .
$$

In the new coordinates the equations of motion become

$$
\dot{y}=B^{-1} A(\theta) B y+B^{-1} u(B y, \theta)-B^{-1} \dot{B} y,
$$

where the second term is the transformed nonlinear part of the vector field, and the last term is the residual of the timedependent transformation. Using the definition of $B$ and $d X / d \theta=A(\theta) X$ we express this last term as

$$
B^{-1} \dot{B}=\left(e^{\Omega \theta} X^{-1}\right)\left(\dot{X} e^{-\Omega \theta}-X e^{-\Omega \theta} \Omega\right)=B^{-1} A(\theta) B-\Omega
$$

(note that $\Omega$ and $\exp \Omega \theta$ commute), so that (30b) becomes indeed

$$
\dot{y}=\Omega y+B^{-1} u(B y, \theta) .
$$

\section{Floquet transformation of a Hamilton function}

In the Hamiltonian case (Hamiltonian matrix $A$ and symplectic matrix $B$ ) the residual matrix $B^{-1} \dot{B}$ is Hamiltonian ([16], Chap. II A, Theorem 3). In other words, if $\mathcal{F}$ is the generating function of (linear) canonical transformation (29c), and $\mathcal{J}$ is the old Hamilton function with nonlinear part $\mathcal{U}=O\left(x^{2}\right)$, then

$$
\begin{gathered}
B^{-1} \dot{B} x=\left(\begin{array}{cc}
0 & I \\
-I & 0
\end{array}\right) \nabla_{x} \frac{\partial}{\partial \theta} \mathcal{F}, \\
B^{-1} A B y+\cdots=\left(\begin{array}{cc}
0 & I \\
-I & 0
\end{array}\right) \nabla_{y} \mathcal{J}(B y),
\end{gathered}
$$

and the new Hamilton function is

$$
\tilde{\mathcal{J}}(y, \theta)=y^{T} \Omega^{T}\left(\begin{array}{cc}
0 & I \\
-I & 0
\end{array}\right) y+\mathcal{U}(B(\theta) y, \theta) .
$$

Therefore, to make transformation (29c) of Hamiltonian $\mathcal{J}_{\varepsilon, E}$ in $(14 \mathrm{~b})$, with $x=\left(p_{\sigma}, \sigma\right)$, we (1) separate $\mathcal{J}_{\varepsilon, E}$ into linear (quadratic in $x$ ), and nonlinear $\left[O\left(x^{2}\right)\right]$ parts; (2) replace the linear part by the time-independent quadratic form with matrix $\Omega$; and (3) substitute $x \rightarrow B(\theta) x$.

\section{Linear stability in the generic case}

As outlined in Sec. II B, the stability of periodic orbits is traditionally analyzed in terms of the multipliers of the monodromy matrix (28d), the linearized Poincaré map. We can do the same using the quadratic part of the reduced Floquet-transformed Hamilton function $\widetilde{\mathcal{J}}$, i.e., the eigenvalues of $\Omega=\Omega_{\varepsilon}$ in (31c). The two approaches apply near the origin (the central orbit), and are, of course, equivalent since eigenvalues of $\Omega_{\varepsilon}$ are characteristic exponents of $M_{\varepsilon}\left[\Omega_{\varepsilon}\right.$ $\in \operatorname{sp}\left(2 N^{\prime}, r\right)$ is the infinitesimal generator of $M_{\varepsilon}$ $\left.\in \operatorname{Sp}\left(2 N^{\prime}, r\right)\right]$.

Once the Hamilton function $\mathcal{J}_{\varepsilon, E}$ is put into form (31c) and its quadratic terms are made time independent, we analyze the stability of its central equilibrium (stationary point) precisely as discussed in Sec. II A 2. $\left[\Omega_{\varepsilon}^{T}\right.$ in Eq. (31c) is the analog of the Hessian matrix in Eqs. (3b) and (A1). If $N^{\prime}=1$, a max or min of $\widetilde{\mathcal{J}}(y)$ is a stable PO while a saddle is an unstable PO.]

For any $N^{\prime}$, elementary orbits correspond to isolated, i.e., nondegenerate stationary points of Hamiltonian (31c) (Secs. II B 1). Thus if the central orbit, which corresponds to the equilibrium at the origin $y=0$, is elementary, the matrix $\Omega_{\varepsilon}$ is nondegenerate [cf. Eqs. (3)]: none of its eigenvalues $i \omega_{j}(\varepsilon)$ vanishes,

$$
\operatorname{det} \Omega_{\varepsilon} \neq 0 \Leftrightarrow \omega_{j} \neq 0, \quad j=1, \ldots, N^{\prime}
$$

in other words, all harmonic frequencies $\omega_{j}$ of Hamiltonian (31c) are nonzero.

These harmonic frequencies $\omega_{j}(\varepsilon)$ in (32) are independent functions of $\varepsilon$, and therefore typically only one of them will pass through zero at some isolated value $\varepsilon_{\text {crit }}\left[\Omega_{\text {crit }}\right.$ has a single nilpotent $2 \times 2$ block $\left(\begin{array}{ll}0 & \beta \\ 0 & 0\end{array}\right)$. The set of degenerate members of the generic family $\Omega_{\varepsilon}$ is of measure zero.] These $\varepsilon_{\text {crit }}$ are the bifurcation points [24,26].

There are three principal types of resonances in the reduced Hamiltonian problem (31c): (i) singularities in the linear problem and corresponding $C_{1}$ and $C_{2}$ (period 1 and 2) bifurcations; (ii) resonances that involve one single mode of (31c) and "time," i.e., the motion along the orbit, and that result in period- $k\left(C_{k}\right), k>2$, bifurcations; (iii) if $N^{\prime}>1$, resonances between different transverse modes of (31c). Types (i) and (ii) are directly related to bifurcations of PO's, and will be studied here. These cases are two-dimensional phenomena and we can use $N^{\prime}=1$ (Secs. VI B and VII A 5) [24].

There remains one problem that is worth mentioning - the absence of a real $\ln M_{\varepsilon}$ in the $C_{2}$ case. Consider the $2 \times 2$ matrices in Appendix D. Domains of real and complex logarithms overlap for $\tau=\frac{1}{2}|\operatorname{Tr} M|<1$. The $C_{1}$ bifurcation occurs at $\tau=1$, at the edge of the complex domain, and therefore can only be analyzed in terms of the real logarithm of $M$ [Eq. (D4c)]. All $k>2$ phenomena happen in the elliptic domain $|\tau|<1$ and again we may use this real logarithm of $M$ [Eq. (D4c)]. On the other hand, the $C_{2}$ bifurcation happens for $\tau=-1$, at the edge of the real domain, so that the complex logarithm must be used.

To understand this situation better it is useful to imagine the topology of the extended phase space $(y, \theta)$, or, in other words, the local topology of the constant energy level set of the initial problem near the periodic orbit. For the real case (the central orbit is stable) our space is foliated as a set of tori. In the complex case the points at the beginning and the end of the period are connected Möbius-wise and the topology is different [39]. To avoid the problem and to have real 


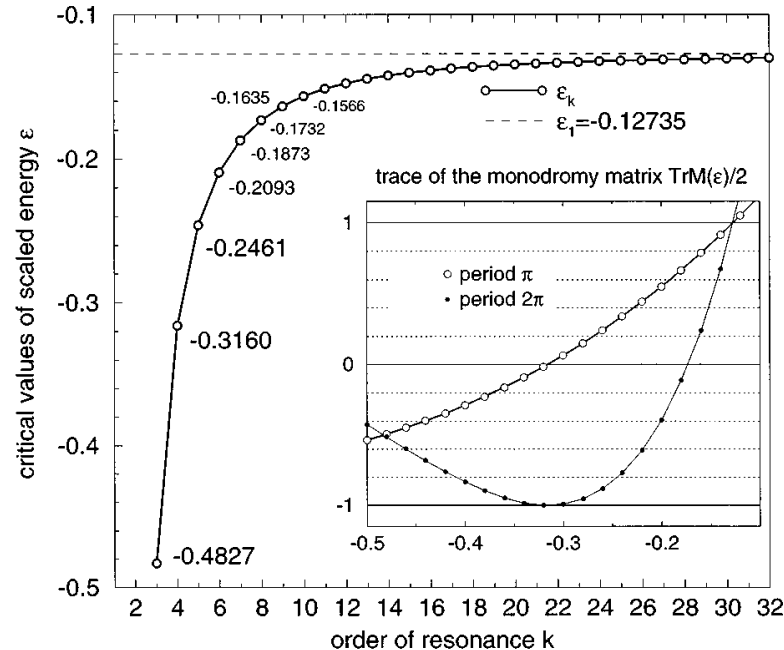

FIG. 9. Critical values of scaled energy for period- $k$ bifurcations of perpendicular orbit [Eq. (33), $n=1]$ and the trace of the monodromy matrix calculated from Mathieu-Hill's equation (circles) and compared to direct numerical calculation (solid lines).

periodic-in- $\theta$ equations we may double the period to $4 \pi$ [two sheet coverage ([17], Sec. 5.26 G)] so that $\ln M^{2}$ is real. The double-period system has $C_{2}$ symmetry and the bifurcation is of type $C_{2}$ with two $C_{2}$-equivalent stationary points connected by $M$.

\section{Floquet analysis for perpendicular trajectory}

Let us now carry out this analysis for the perpendicular $\mathrm{PO}$ of the diamagnetic Kepler problem. We obtain the linearization of the equations in normal variations defined by (21) by retaining only terms of type $J_{\varepsilon, 01}^{0}$ (there are no other purely quadratic in $p_{\sigma}$ terms) and $J_{\varepsilon, 10}^{l}$. Their coefficients are shown in Fig. 7. To compute a fundamental matrix solution $X_{\varepsilon}(\theta)$ we use numerical integration of the linearized equations for two initial conditions $x_{1}(0)=(1,0)$ and $x_{2}=(0,1)$ with $x=\left(\sigma, p_{\sigma}\right)$. The resulting column vectors $x_{1}(\theta)$ and $x_{2}(\theta)$ form the matrix $X_{\varepsilon}(\theta)$.

\section{The half-period monodromy matrix}

As explained in Sec. IV C 3 the reduced Hamiltonian for the perpendicular orbit (21) is $\pi$ periodic in $\theta$ due to the $\left(\mathrm{R}_{\lambda} \sim C_{*}\right)$ invariance of the orbit. As a consequence, the monodromy matrix (as well as the Poincare map itself) can be defined at $\pi$, the first return time, $M_{\varepsilon}^{(\pi)}=X_{\varepsilon}(\pi)$, instead of at $2 \pi$, the actual period of the orbit. This circumstance has been exploited by Mao and Delos [6] who computed the "half map" by registering all crossings of the surface of section (regardless of direction). Using the half period we should, however, remember how the halves are connected into complete trajectories (see Appendix F).

The trace of $M_{\varepsilon}^{(\pi)}$ is used to determine critical values $\varepsilon_{\text {crit }}^{n, k}$ at which an $(n, k)$ bifurcation of the half map occurs:

$$
\tau\left(\varepsilon_{\text {crit }}^{n, k}\right)=\frac{1}{2} \operatorname{Tr} M^{(\pi)}\left(\varepsilon_{\text {crit }}^{n, k}\right)=\cos (2 \pi n / k) .
$$

This trace, and the associated solutions to Eq. (33), are shown in Fig. 9. The trace is a monotonic function of $\varepsilon$ : as

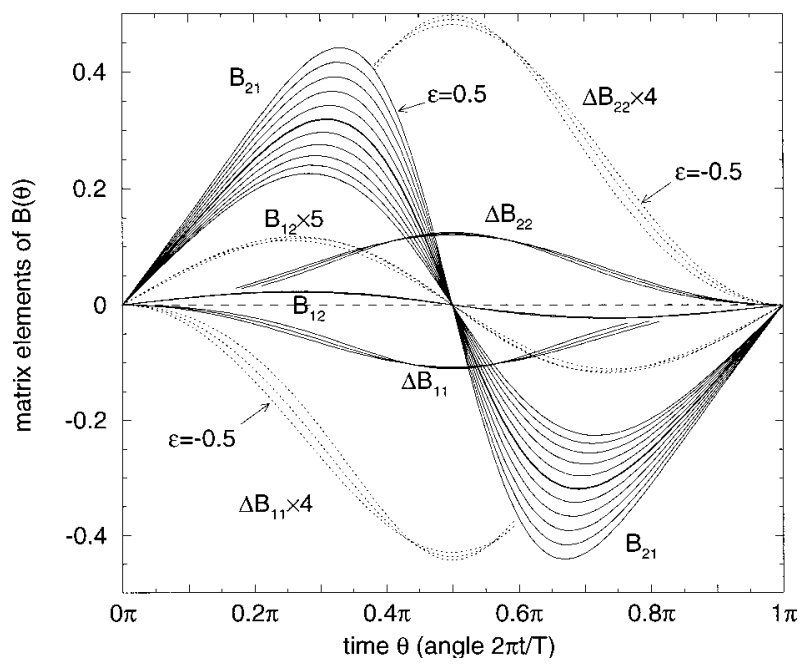

FIG. 10. Matrix of the Floquet transformation $B(\theta)$ in Eqs. (29) at different scaled energies $-0.5 \leqslant \varepsilon \leqslant 0.5$ for perpendicular trajectory $[\Delta B(\theta)=B(\theta)-I]$.

$\varepsilon$ increases $\operatorname{Tr} M$ passes critical values with $k=3,4,5, \ldots$, and then 1 ( $k=2$ "occurs" for $\varepsilon \rightarrow-\infty)$.

\section{Floquet transformation}

Since in our case $\tau>-1$ (Fig. 9) we can always use a real logarithm (Appendix D) and this simplifies the analysis. Obviously, $B$, the matrix of the Floquet transformation (29), has the same period as Hamiltonian (21), i.e., because of symmetry $\mathrm{R}_{\lambda}$ the the matrix $B_{\varepsilon}(\theta)$ has period $\pi$. We define $\widetilde{\theta}=2 \theta$, so that $B_{\varepsilon}(\widetilde{\theta})$ is $2 \pi$ periodic in $\widetilde{\theta}$ and is represented as a Fourier series on the interval $0 \leqslant \widetilde{\theta}<2 \pi$. Due to the initial condition $X(0)=I$ matrix elements of $B-I$ can be cast in terms of pure sine or cosine (without constant term) series as follows:

$$
B_{\varepsilon}(0)=\left(\begin{array}{ll}
1 & 0 \\
0 & 1
\end{array}\right), \quad B_{\varepsilon}(\widetilde{\theta})=\left(\begin{array}{cc}
{[\cos ]} & {[\sin ]} \\
{[\sin ]} & {[\cos ]}
\end{array}\right)
$$

where $[\sin ]$ and $[\cos ]$ mean Fourier sine and Fourier cosine series,

$$
[f](\theta)=\sum_{l \geqslant 0} F_{\varepsilon}^{(l)} f(l \theta), \quad f=\sin \text { or } \cos ,
$$

$F^{(0)} \equiv 0$ (i.e., $\left.l \geqslant 1\right)$ in the sine series and $F^{(0)}=1$ in the cosine series. These matrix elements are shown in Fig. 10.

As outlined in Sec. V B 3 we transform Hamiltonian (21) by substituting $x=\left(\sigma, p_{\sigma}\right)$ for $B y$ in all nonlinear terms. Two coordinate representations of the result of this substitution are required for the subsequent normal form reduction. Below we present the essentials of these results in the simplified Fourier series notation (34b) with amplitudes $F^{(l)}$ as well as orders $l$ specified separately. To follow note that in the spirit of this notation

$$
[\sin ][\sin ]=[\cos ][\cos ]=[\cos ], \quad[\sin ][\cos ]=[\sin ] .
$$


a. Polar coordinates. If the matrix $\Omega$ of the linear equations (29d) is nonsingular (i.e., for cases $k>2$ in Table I) we use action-angle coordinates such that

$$
x=\left(\begin{array}{c}
\sigma \\
p_{\sigma}
\end{array}\right)=B_{\varepsilon}(\widetilde{\theta}) \sqrt{2 I}\left(\begin{array}{c}
\sin \varphi \\
\cos \varphi
\end{array}\right)=B_{\varepsilon}(\widetilde{\theta}) y .
$$

In these coordinates our reduced Hamiltonian becomes a combination of Fourier series:

$$
\mathcal{J}_{\varepsilon}=\omega I+[\cos ](2 \varphi)[\cos ](\widetilde{\theta})+[\sin ](2 \varphi)[\sin ](\widetilde{\theta})
$$

Both $\varphi$ and $\widetilde{\theta}$ series start with $l=1$ for sines, and with $l=0$ for cosines; the $\varphi$ series has the form

$$
\sum_{l=1}(2 I)^{l} \sum_{s=0} F_{\varepsilon, s}^{(l)}(2 I)^{s} \cos (2 l \varphi)
$$

To understand the origin of Eqs. (35b) and (35c) consider nonlinear terms in Eq. (21), such as

$$
\sigma^{4}=\left\{b_{11}[\cos ](\widetilde{\theta}) \cos (\varphi)+b_{12}[\sin ](\widetilde{\theta}) \sin (\varphi)\right\}^{4},
$$

use Eq. (34c), and verify that these terms indeed are $\pi$ periodic in $\varphi$ and $2 \pi$ periodic in $\widetilde{\theta}$. In fact, the $\pi$ periodicity in $\varphi$ is due to the $\mathrm{R}_{\sigma}$ symmetry: (21) is an even function of both $\sigma$ and $p_{\sigma}$. On the other hand, since we use a half map $M^{(\pi)}$ with time $\widetilde{\theta}=2 \theta$, our problem no longer possesses any special property with regard to $R_{\lambda}$. (In other words, we reduced our problem with regard to $R_{\lambda}$.)

In the actual numerical procedure that we have developed, this Floquet transformation is done at a series of fixed values of parameter $\varepsilon$ near the critical value $\varepsilon_{\text {crit }}$ obtained in the linear study (Fig. 9). ( $\varepsilon$ dependence is reintroduced only after a collection of normal forms at various fixed $\varepsilon$ is obtained.) The old coordinates $x=\left(\sigma, p_{\sigma}\right)$ in the Hamilton function (21) are replaced by the new coordinates $y$ such that

$$
x=B(\theta) S_{\alpha} y=B(\theta) S_{\alpha}\left(\begin{array}{c}
\rho \sin \varphi \\
\rho \cos \varphi
\end{array}\right),
$$

with $S_{\alpha}=\operatorname{diag}\left(\alpha^{-1}, \alpha\right)$ a scaling matrix to have the quadratic part in (31c) in a standard form $\frac{1}{2} \omega y^{T} y$. To actually express (21) in terms of $y$ the values of $\mathcal{J}_{\varepsilon}\left(p_{\sigma}(y, \theta), \sigma(y, \theta) ; \theta\right)$ are sampled over a grid in polar coordinates $\rho, \varphi$, and "time", $\theta$. The coefficients $\mathcal{J}_{s, f}^{(k, n)}$ in the Fourier representation (35b) of $\mathcal{J}$,

$$
\begin{gathered}
\mathcal{J}(y ; \theta)=\frac{1}{2} \omega \rho^{2}+\rho^{4} \sum_{k, n, s, f} \mathcal{J}_{s, f}^{(k, n)} \rho^{2 s} f(2 k \varphi) f(n \theta), \\
s \geqslant k-2, \quad f=\sin \text { or cos, }
\end{gathered}
$$

with $(k, n)$ integers 0 (if $f=\cos$ ), $1,2, \ldots$, are subsequently obtained from a Fourier transform in $\theta$ and $\varphi$ and a polynomial fit in $\rho^{2}$. Table III gives an example of these coefficients.

b. Rectangular coordinates. If the linear part is singular the problem is essentially one dimensional and we keep rectangular coordinates $y$. It is, however, convenient to work
TABLE III. Leading terms $\mathcal{J}_{s, f}^{(k, n)}$ in the nonlinear part of the Floquet-transformed Hamilton function (35) for $\varepsilon=-0.31628$ (at the period-4 bifurcation). Notation as in Eq. (36b) with $\alpha=1.0865$ and $\omega=\frac{1}{4}$.

\begin{tabular}{ccccccc}
\hline \hline$k, n$ & $f$ & $10^{-5} \rho^{8}$ & $10^{-4} \rho^{6}$ & $10^{-3} \rho^{4}$ & $10^{-2} \rho^{2}$ & $10^{-2} \rho^{0}$ \\
\hline 0,0 & $\cos$ & 33.245 & 20.209 & 29.049 & 10.057 & -2.7210 \\
1,0 & $\cos$ & -24.049 & -21.335 & -44.672 & -14.713 & 6.0584 \\
2,0 & $\cos$ & -32.437 & -8.1164 & 19.946 & 5.5598 & -1.9909 \\
3,0 & $\cos$ & 28.533 & 14.670 & -4.8630 & -0.9033 & \\
4,0 & $\cos$ & -1.3781 & -6.3843 & 0.5400 & & \\
5,0 & $\cos$ & -5.4510 & 0.9567 & & & \\
6,0 & $\cos$ & 1.5367 & & & & \\
0,1 & $\cos$ & -16.209 & -4.1809 & 7.9024 & 4.9031 & 4.7888 \\
1,1 & $\cos$ & 11.658 & 4.4147 & -12.978 & -7.6133 & -7.3363 \\
2,1 & $\cos$ & 15.937 & 1.4335 & 6.7353 & 3.2879 & 2.2397 \\
3,1 & $\cos$ & -13.937 & -2.4857 & -1.8001 & -0.5779 & \\
4,1 & $\cos$ & 0.5937 & 0.9152 & 0.1402 & & \\
5,1 & $\cos$ & 2.7229 & -0.0968 & & & \\
6,1 & $\cos$ & -0.7650 & & & & \\
1,1 & $\sin$ & -58.825 & -24.312 & -5.0572 & -1.1582 & -0.6157 \\
2,1 & $\sin$ & 42.835 & 24.546 & 4.4981 & 0.8201 & -0.0393 \\
3,1 & $\sin$ & 2.2239 & -10.913 & -1.5728 & -0.1711 & \\
4,1 & $\sin$ & -15.347 & 2.1211 & 0.1947 & & \\
5,1 & $\sin$ & 6.6388 & -0.1050 & & & \\
6,1 & $\sin$ & -0.8866 & & & & \\
0,2 & $\cos$ & -6.8055 & -3.3590 & -3.8895 & -0.8140 & -0.1055 \\
1,2 & $\cos$ & 8.3255 & 5.3001 & 6.2688 & 1.2820 & 0.3812 \\
2,2 & $\cos$ & 1.2230 & -2.3281 & -3.1390 & -0.5677 & -0.2516 \\
3,2 & $\cos$ & -5.6703 & 0.1938 & 0.8366 & 0.0993 & \\
4,2 & $\cos$ & 4.2177 & 0.2845 & -0.0769 & & \\
5,2 & $\cos$ & -1.5258 & -0.0913 & & & \\
6,2 & $\cos$ & 0.2354 & & & & \\
1,2 & $\sin$ & 21.160 & 5.1828 & 0.2569 & -0.0105 & 0.1254 \\
2,2 & $\sin$ & -15.665 & -5.2228 & -0.0709 & 0.0525 & 0.0273 \\
3,2 & $\sin$ & -0.5131 & 2.3199 & -0.0809 & -0.0279 & \\
4,2 & $\sin$ & 5.4834 & -0.4549 & 0.0320 & & \\
5,2 & $\sin$ & -2.4568 & 0.2462 & & & \\
6,2 & $\sin$ & 0.3434 & & & & \\
\hline \hline & & & & & & \\
\hline
\end{tabular}

with an imaginary Hamiltonian. This requires a symplectic transformation with multiplier $i$, such that

$$
x=\left(\begin{array}{c}
\sigma \\
p_{\sigma}
\end{array}\right)=B_{\varepsilon}(\theta) S_{\alpha}\left(\begin{array}{c}
q \\
i p
\end{array}\right)=B_{\varepsilon}(\theta) S_{\alpha} y .
$$

This time $\alpha$ is chosen to put the $p_{\sigma}^{2}$ term in the standard form $p^{2} / 2$.

\section{IDEA OF LIE TRANSFORMATIONS}

We return again to general theory. In the previous section we have used an exact change of variables to make the linear part of our problem time independent; i.e., we converted Eq. (30a) containing $A(\theta)$ to (30d) containing $\Omega$ independent of $\theta$. For the purposes of this section, let us temporarily suppress the $\theta$ dependence that remains in the nonlinear terms, 
and consider a general autonomous set of equations having the form

$$
\dot{x}=A x+u(x) .
$$

The matrix $A$ can now be used to construct a formal nearidentity change of variables

$$
x=y+\mathfrak{w}(y)=y+O\left(|y|^{2}\right),
$$

which preserves the linear part of (38), but which reduces the nonlinear part to a "minimal" or "normal" form.

Let us introduce a formal small parameter $\epsilon$, such that

$$
\dot{x}=A x+\epsilon u(x)+\cdots,
$$

and consider a change of variables

$$
x=y+\epsilon w(y), \quad w(y) \sim O\left(|y|^{2}\right) .
$$

Ideally, Eq. (40a) might be reduced to a purely linear [up to an arbitrary high order $\left.O\left(|y|^{s}\right)\right]$ equation. This, however, is often impossible; instead the normal form of Eq. (40a),

$$
\dot{y}=A y+\epsilon v(y)+\cdots,
$$

retains nonlinear terms $v(y)$ that no transformation of type (39) can eliminate. Such terms are the resonance terms (cf. Sec. II B 5), and they always exist in Hamiltonian systems (see Sec. VII A 4). To find the resonance terms $v$ and the corresponding generator $w$, in other words to shape the strategy of the transformation, we study in the following section the infinitesimal case $\epsilon \rightarrow 0$ with all higher orders neglected.

The finite transformation (operation of a continuous group) for $\epsilon \neq 0$, now written as $y+\mathfrak{w}(y, \epsilon)$ in Eq. (39), is a solution of the equation

$$
\frac{\partial \mathfrak{w}(y, \boldsymbol{\epsilon})}{\partial \boldsymbol{\epsilon}}=w(y+\mathfrak{w}(y, \boldsymbol{\epsilon}), \boldsymbol{\epsilon}), \quad \mathfrak{w}(y, 0)=0,
$$

defined by the generator (operator of the corresponding algebra) $w(y, \boldsymbol{\epsilon})$. In other words,

$$
\begin{aligned}
\mathfrak{w}(y, \boldsymbol{\epsilon}) & =\left.\epsilon \frac{\partial \mathfrak{w}(y, \boldsymbol{\epsilon})}{\partial \boldsymbol{\epsilon}}\right|_{\epsilon=0}+\left.\epsilon^{2} \frac{\partial^{2} \mathfrak{w}(y, \epsilon)}{\partial \epsilon^{2}}\right|_{\boldsymbol{\epsilon}=0}+\cdots \\
& =\epsilon w(y, 0)+\cdots
\end{aligned}
$$

Clearly, to study the transformation of coordinates we need to construct $\mathfrak{w}(y, \boldsymbol{\epsilon})([40]$, Chap. 12.2, Theorem 2.2). However, it turns out that to obtain the new equations of motion (41) we only need the generator $w(y, \boldsymbol{\epsilon})$.

We will see that reduction to a normal form is a stepwise perturbation technique whose zero order, the linear part $A x$, remains unchanged, while higher orders are sequentially reduced. The transformation accumulates: reduction of order $r$ contributes to orders $s>r$. The main advantage of the Lie transformation theory is that it gives a very efficient way to keep track of this accumulating transformation.

\section{A. Algorithm of Lie transformation}

\section{Lie equation}

Let us first focus on the principal aspect of the required transformation and consider an infinitesimal $(\epsilon \rightarrow 0)$ formal near identity change of variables. Substituting (40b) into (40a) gives

$$
\begin{aligned}
\dot{y} & =A y+\epsilon A w(y)-\frac{d w}{d y} \epsilon \dot{y}+\epsilon u[y+\epsilon w(y)] \\
& =A y+\epsilon\left[A w(y)-\frac{d w}{d y} A y\right]+\epsilon u(y)+\cdots,
\end{aligned}
$$

where the derivative of $w$ is a matrix

$$
\frac{d}{d y}\left(\begin{array}{c}
w_{1} \\
w_{2} \\
\vdots
\end{array}\right)=\left(\begin{array}{c}
\nabla_{y} w_{1} \\
\nabla_{y} w_{2} \\
\vdots
\end{array}\right)=\left(\begin{array}{ccc}
\frac{\partial w_{1}}{\partial y_{1}} & \frac{\partial w_{1}}{\partial y_{2}} & \ldots \\
\frac{\partial w_{2}}{\partial y_{1}} & \frac{\partial w_{2}}{\partial y_{2}} & \ldots \\
\vdots & \vdots & \ddots
\end{array}\right)
$$

Ideally we would like to put Eq. (40a) in a purely linear [up to $\left.O\left(\epsilon^{2}\right)\right]$ form $\dot{y}=A y+\cdots$, i.e., to eliminate all nonlinear terms of order $\epsilon$. As seen from (43a) this requires solving the homological or Lie equation ([17], Chap. 5, Sec. 22C)

$$
\mathfrak{L}_{A} w(y)=\frac{d w}{d y} A y-A w(y)=u(y) .
$$

However, as was already known to Poincaré, the solution $w(y)$, the generator of the desired transformation, does not always exist, so that in general the normal form (41) contains some residual nonlinearity $v(y)$.

\section{Finite transformation}

The Lie transformation technique provides an efficient algorithm to accumulate (integrate) transformations. If the generator $w(y, \epsilon),[41]$ the initial nonlinearity $u$, and the final nonlinearity $v$ are expressed as formal Taylor series in $\epsilon$,

$$
\begin{gathered}
w(x, \epsilon)=\sum_{k=0} \frac{\epsilon^{k}}{k !} w_{k+1}(x), \\
u(x, \epsilon)=\sum_{r=0} \frac{\epsilon^{r}}{r !} u_{r}^{0}(x), \\
v(x, \epsilon)=\sum_{r=0} \frac{\epsilon^{r}}{r !} u_{0}^{r}(x),
\end{gathered}
$$

we find the terms in $v(y, \epsilon)$ by an iterative procedure:

$$
u_{j}^{i}=u_{j+1}^{i-1}+\sum_{k=0}^{j}\left(\begin{array}{l}
j \\
k
\end{array}\right) \mathfrak{L}\left(u_{j-k}^{i-1}, w_{k+1}\right) .
$$

(Proof is in Ref. [16], Chap. VII A2 or Ref. [40], Chap. 12.2.) The operation $\mathfrak{L}$ is often called the Lie product or Lie bracket. For vector fields it is a commutator 


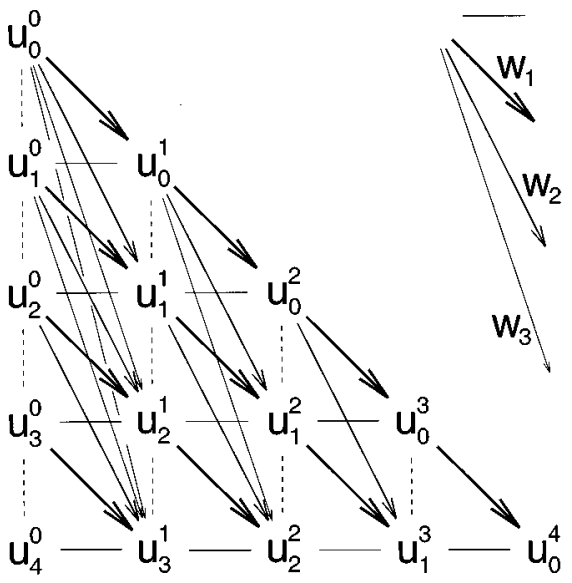

FIG. 11. Lie triangle.

$$
\mathfrak{L}(u, w)=\frac{d u}{d x} w-\frac{d w}{d x} u
$$

with derivatives as in Eq. (43b). Thus operation $\mathfrak{L}_{A}$ is nothing else but

$$
\mathfrak{L}_{A} w(x)=-\mathfrak{L}(A x, w)=-\mathfrak{L}\left(u_{0}^{0}, w\right) .
$$

The implementation of Eq. (44d) is best illustrated by the so-called Lie triangle in Fig. 11. Rows $r=0$ (the top), 1, 2, etc., of this triangle correspond to the order- $r$ steps of the algorithm. Each row has $r+1$ terms (columns $i=0, \ldots, r)$; the leftmost $(i=0)$ and rightmost $(i=r)$ terms are the initial $u_{r}^{0}$ and the transformed $v_{r}=u_{0}^{r}$. The calculation proceeds with increasing $r$ (down from the top), and $i$ (from left to right on each row, $u_{r}^{0} \rightarrow u_{0}^{r}$ ) and involves (if $r>1$ ) intermediate terms $u_{j}^{i}$ with $i+j=r$. To calculate a term $i>0$ in a row we use already known terms in the column $i-1$ on the immediate left. We take the left term on the current row [horizontal line, first term in the right-hand side of Eq. (44d)] and add contributions $\mathfrak{L}(u, w)$ due to generators $w_{k}$ with $k=1, \ldots, r-i+1$ combined with terms $u$ in the left column above our row [arrows from up left, sum in the right-hand side of Eq. (44d)].

The important point to notice is about the generators involved. The lowest-order contribution due to $w_{r}$ is of order $\boldsymbol{\epsilon}^{r} \quad$ the order of $\mathfrak{L}$ terms in Eq. (44d) is $(i-1)+(j-k)+(k+1)=r$, cf. Eq. (42)] and therefore the effect of $w_{r}$ begins on row $r$ (for transformation of order $\epsilon^{r}$ ) where it contributes only to the term $u_{r-1}^{1}$ in the $i=1$ column. This main contribution by $w_{r}$, which is, of course, due to $\mathfrak{L}_{A} w_{r}[i=1, k=j=r-1$ in Eq. (44d)], can be used to eliminate some of the terms of order $r$. To find $w_{r}$ we first assume $w_{r}=0$ and proceed along the $r$ th row using generators of orders $1, \ldots, r-1$, already known from previous rows, to calculate $\tilde{u}_{r}$. (The latter is the sum of $u_{r}^{0}$, the initial term of order $r$, and all terms accumulated due to previous transformations.) Next we try to solve the Lie equation (43c) as

$$
\mathfrak{L}_{A} w_{r} \stackrel{?}{=} \tilde{u}_{r} .
$$

Once $w_{r}$ is found we add its contribution $-\mathfrak{L}_{A} w_{r}$ to all terms in the row, ending with

$$
v_{r}=\tilde{u}_{r}-\mathfrak{L}_{A} w_{r} .
$$

This brings us back to the central problem of the theorysolving the Lie equation with given $A$ and $\tilde{u}$.

\section{Solving the Lie equation. Homogeneous polynomials}

The key to Eq. (43c) is that operator $\mathfrak{L}_{A}$ has invariant subspaces $\mathcal{P}\left(y^{m}\right)$ spanned by homogeneous polynomials of the same total power $m$, i.e., $\mathfrak{L}_{A}: \mathcal{P}\left(y^{m}\right) \rightarrow \mathcal{P}\left(y^{m}\right)$. Moreover, $\mathfrak{L}_{A}$ is a linear operator on $\mathcal{P}\left(y^{m}\right)$ with homogeneous monomials forming a convenient basis. If the null space (kernel) of $\mathfrak{L}_{A}$ restricted to $\mathcal{P}\left(y^{m}\right)$ is not empty, then the normal form $v(y)$ retains monomials in this null space, $\operatorname{Ker} \mathfrak{L}_{A} \mid \mathcal{P}\left(y^{m}\right)$. As we will see below in Eq. (52c), precisely this happens if there exist resonances of order $m$. All other monomials in the range space (image) of $\mathfrak{L}_{A}\left(\tilde{u}_{m} \in \operatorname{Im} \mathfrak{L}_{A}\right)$, i.e., all nonresonant terms, can be eliminated by a proper choice of generators $w_{m} \in \operatorname{Im} \mathfrak{L}_{A}$.

Following these ideas we represent $w_{r}, v_{r}=u_{0}^{r}$, and $u_{r}=u_{r}^{0}$ in Eqs. (44) and all other entries in the Lie triangle as vector-valued polynomials in $x$, such as

$$
u_{j}^{i}(x)=\sum_{\{m\}}\left(\begin{array}{c}
\left(u_{j}^{i}\right)_{1}^{\{m\}} \\
\left(u_{j}^{i}\right)_{2}^{\{m\}} \\
\vdots \\
\left(u_{j}^{i}\right)_{N}^{\{m\}}
\end{array}\right) x^{\{m\}},
$$

where $x^{\{m\}}$ is a monomial,

$$
x^{\{m\}}=x_{2}^{m_{2}} x_{2}^{m_{2}} \cdots x_{N}^{m_{N}},
$$

the sum is over all possible monomials of fixed degree

$$
\{m\}=\left\{m_{1}, m_{2}, \ldots, m_{N} \mid m_{i} \geqslant 0, \sum_{i=1}^{N} m_{i}=m\right\},
$$

and the well-decorated $\left(u_{j}^{i}\right)_{k}^{\{m\}}$ in Eq. (47a) are coefficients.

The relation between the power $r$ of the formal parameter $\epsilon$ and the degree $m$ of the polynomial depends on the particular problem. Typically, in a Taylor series, the natural correspondence is $r=i+j$ and $m=r+1$, specifically, $r=0$ and $m=1$ for $A x$. In some cases (with symmetry) we may have $m=2 r+1$.

Further details of the implementation of the algorithm depend on whether or not the matrix $A$ can be brought to diagonal form. These two cases have to be treated separately; we consider them in Sec. VII, where we also focus on the application of the theory to Hamiltonian ordinary differential equations (ODE's).

\section{B. Nonautonomous equations with periodic coefficients}

Application of the above theory to our problem, reduction of nonautonomous equations of type (30d) to a normal form, needs further analysis. There are several ways to go, but in 
any case we should take advantage of the fact that the time dependence of (30d) is $2 \pi$ periodic (cf. Appendix E 2).

A direct way to take immediate advantage of the periodicity in $\theta$ is to introduce a supplementary pair of variables [31]

$$
x=\left(\begin{array}{c}
\sin \theta \\
\cos \theta
\end{array}\right), \quad \dot{x}=\left(\begin{array}{cc}
0 & 1 \\
-1 & 0
\end{array}\right) x
$$

or, equivalently,

$$
\zeta=\left(\begin{array}{c}
\exp (+i \theta) \\
\exp (-i \theta)
\end{array}\right), \quad \dot{\zeta}=\left(\begin{array}{cc}
i & 0 \\
0 & -i
\end{array}\right) \zeta
$$

We add two new differential equations, (48a) or (48b), to our original system, and replace all $\theta$-periodic factors by their expression in terms of $x$ or $\zeta$. [Recall our change $\theta \leftrightarrow\left(q_{2}, p_{2}\right)$ in Eq. (10b) [31].] The resulting system (which is now autonomous) can be put into normal form by the algorithm already developed above. Moreover, the two added oscillator equations are already linear and are not transformed ("time" is not being redefined) - their variables and their matrices just participate in the transformation of other equations. In other words, the corresponding components of the generator $w$ are always 0 .

In our application to periodic orbits, we go from $2 \mathrm{~N}$ autonomous Hamiltonian equations to $2 N-2$ nonautonomous Hamiltonian equations (isoenergetic reduction), then back to $2 N$ autonomous but non-Hamiltonian equations. The $2 N-2$ equations describe oscillations transverse to the orbit and driven by a periodic force (due to the changing potential along the orbit). The $2 N$ equations include this force (i.e., the motion along the orbit) as a dynamical subsystem. The latter, however, remains independent from the $2 N-2$ subsystem: it pushes without response. It is the resonances between the two subsystems, the driving and the driven, that are responsible for the bifurcations of the central orbit. We consider only the resonances of this kind. (In the generic situation these resonances occur isolated from each other and from those among the modes of the $2 N-2$ subsystem.)

\section{SYMPLECTIC LIE TRANSFORMATIONS. NORMAL FORMS OF HAMILTONIAN VECTOR FIELDS}

While it may seem simpler to work with Hamilton functions (Appendix E 1), the advantage becomes marginal for an algebraic processor or a computer program-in particular if the transformation depends periodically on time $\theta$. We implement the general (time-independent) vector field algorithm in Secs. VI A and VI B, converting the differential equations to normal form, and then we rewrite the result as a new Hamilton function. This algorithm may or may not automatically preserve the symplectic structure of our initial equations of motion. Even if it does not, we can make this algorithm symplectic by requiring the generating field $w(x, \theta, \epsilon)$, the solution of Lie equation (43c), to be Hamiltonian. More precisely, we require the $2 N-2$ components of $w$ to be Hamiltonian when the two added variables (48a) or (48b) are treated as parameters. The way to do this as well as to solve Eq. (43c) depends on the linear part.

\section{A. Normal form in the diagonal case}

Consider a case where the matrix $A$ of the linear part of (38) can be diagonalized, let $\left\{\lambda_{1}, \lambda_{2}, \ldots, \lambda_{N}\right\}$ be the eigenvalues of $A$, and take our equations already in the diagonal form

$$
\dot{z}=A z+u(z)=\left(\lambda_{1} z_{1}, \lambda_{2} z_{2}, \ldots, \lambda_{N} z_{N}\right)^{T}+u(z) .
$$

Since the field is Hamiltonian, Eqs. (49) are canonical. We will use three different types of canonical variables: complex $(z, \bar{z})$, action angle or polar $(I, \varphi)$, and the usual coordinate and momentum $(q, p)$. They can be defined as follows:

$$
\begin{aligned}
(q, p) & =(\sqrt{2 I} \sin (\varphi), \sqrt{2 I} \cos (\varphi)) \\
& =\left(\frac{1}{2}(z+\bar{z}), \frac{1}{2} i(z-\bar{z})\right),
\end{aligned}
$$

with indices such that

$$
z_{2 s-1}=\bar{z}_{2 s}=-i \sqrt{2 I_{s}} \exp \left(i \varphi_{s}\right), \quad s=1, \ldots, N .
$$

In the above notation the Hamilton function of Eqs. (49) is [30]

$$
H=i \sum_{s=1}^{N} \omega_{s} z_{2 s} \overline{z_{2 s}}+\cdots,
$$

with $N$ harmonic frequencies $\omega$, such that

$$
\lambda_{2 s}=\bar{\lambda}_{2 s-1}=-i \omega_{s} .
$$

We say that these frequencies satisfy a resonance condition if there is a set of integers $m_{s}, s=1, \ldots, N$ such that

$$
(m, \omega)=\sum_{s=1}^{N} m_{s} \omega_{s}=0
$$

and

$$
M=\sum_{i=1}^{N}\left|m_{i}\right|>2 .
$$

For $\lambda, \bar{\lambda}$ we define

$$
\begin{gathered}
(m, \lambda)=\sum_{j=1}^{2 N} m_{j} \lambda_{j}=\sum_{s=1}^{N}\left(m_{2 s-1}-m_{2 s}\right) i \omega_{s}, \\
m_{j} \geqslant 0, \quad M=\sum_{j=1}^{2 N} m_{j}>2 .
\end{gathered}
$$

Note that for vector fields ([17,29], Appendix 7.E) the resonance condition is often written as $\left(m^{\prime}, \lambda\right)=\lambda_{r}$, with $m_{j}^{\prime} \geqslant 0$ as in (52c) and $M^{\prime} \geqslant 2$ [42].

\section{Solving the Lie equation}

Let $\left\{\mathbf{e}_{1}, \mathbf{e}_{2}, \ldots, \mathbf{e}_{N}\right\}$ be the eigenvectors of $A$ and define monomials $z^{\{m\}}$ as in Eqs. (47c) and (47b). These monomials combined with the eigenvectors $\mathbf{e}_{k}$ provide basis vectors on 
the space of vector valued polynomials of total order $m$,

$\mathbf{e}_{k}^{\{m\}}(z)=z^{\{m\}} \mathbf{e}_{k}=(\underbrace{0, \ldots, 0}_{k-1}, z^{\{m\}}, \underbrace{0, \ldots, 0}_{N-k})^{T}$.

These basis vectors (53) are the eigenvectors of $\mathfrak{L}_{A}$ :

$$
\mathfrak{L}_{A} \mathbf{e}_{k}^{\{m\}}(z)=[(\lambda, m)-A] \mathbf{e}_{k}^{\{m\}}(z)=\left[(\lambda, m)-\lambda_{k}\right] \mathbf{e}_{k}^{\{m\}}(z),
$$

since

$$
\begin{aligned}
\left(\frac{d}{d z} \mathbf{e}_{k}^{\{m\}}\right) A z & =z^{\{m\}}\left(\begin{array}{ccc}
k-1 & \text { zero } & \text { rows } \\
\frac{m_{1}}{z_{1}} \frac{m_{2}}{z_{2}} & \cdots & \frac{m_{N}}{z_{N}} \\
N-k & \text { zero } & \text { rows }
\end{array}\right)\left(\begin{array}{c}
\lambda_{1} z_{1} \\
\lambda_{2} z_{2} \\
\vdots \\
\lambda_{N} z_{N}
\end{array}\right) \\
& =\left(\lambda_{1} m_{1}+\lambda_{2} m_{2}+\cdots+\lambda_{N} m_{N}\right) \mathbf{e}_{k}^{\{m\}} \\
& =(\lambda, m) \mathbf{e}_{k}^{\{m\}} .
\end{aligned}
$$

Consequently, eigenvectors $\mathbf{e}_{k}^{\{m\}}(z)$ whose eigenvalues $\left[(\lambda, m)-\lambda_{k}\right]$ equal zero form the basis of the null space $\operatorname{Ker} \mathfrak{L}_{A} \mid \mathcal{P}\left(z^{m}\right)$. All others form the basis of the range space $\operatorname{Im} \mathfrak{L}_{A} \mid \mathcal{P}\left(z^{m}\right)$. This makes solving the Lie equation (46a) straightforward: for each order of the transformation $r=1,2, \ldots$, the solution is

$$
w_{r}=-\sum_{\{m\}} z^{\{m\}}\left(\begin{array}{c}
\left(\tilde{u}_{r}\right)_{1}^{\{m\}} \bar{\Lambda}_{1}^{\{m\}} \\
\left(\tilde{u}_{r}\right)_{2}^{\{m\}} \bar{\Lambda}_{2}^{\{m\}} \\
\vdots \\
\left(\tilde{u}_{r}\right)_{N}^{\{m\}} \bar{\Lambda}_{N}^{\{m\}}
\end{array}\right),
$$

with $\bar{\Lambda}$ 's being the inverted eigenvalues of $\mathfrak{L}_{A}$,

$$
\bar{\Lambda}_{k}^{\{m\}}= \begin{cases}{\left[(\lambda, m)-\lambda_{k}\right]^{-1}} & \text { if }(\lambda, m) \neq \lambda_{k} \\ 0, & \text { otherwise. }\end{cases}
$$

This $w_{r}$ safely eliminates all terms $\widetilde{u_{r}}$ in the range space of $\mathfrak{L}_{A}$, leaving only those in the null space.

\section{Symplectic property}

Solution (56) has been obtained for arbitrary vector fields. If the initial field $u(z)$ is Hamiltonian, we want to preserve this property in $v(z)$. In the diagonal case this will happen automatically.

To prove this we only have to make sure that the generating field $w(z)$ is also Hamiltonian, such as

$$
w_{r}=\left(\begin{array}{c}
\left(w_{r}\right)_{1} \\
\left(w_{r}\right)_{2}
\end{array}\right)=i\left(\begin{array}{c}
(a+1)^{-1} z_{1}^{a+1} z_{2}^{b} \\
-(b+1)^{-1} z_{1}^{a} z_{2}^{b+1}
\end{array}\right),
$$

where $\left(z_{1}, z_{2}\right)$ are canonically conjugate variables. In other words, in the above example we require

$$
\left(w_{r}\right)_{1}^{\{a+1, b\}}=-\left(w_{r}\right)_{2}^{\{a, b+1\}}=\nu .
$$

Since the latter requirement is already fulfilled for $\tilde{u_{r}}$ :

$$
\left(\tilde{u}_{r}\right)_{1}^{\{a+1, b\}}=-\left(\tilde{u}_{r}\right)_{2}^{\{a, b+1\}}=\mu,
$$

and, clearly,

$$
\bar{\Lambda}_{1}^{\{a+1, b\}}=\bar{\Lambda}_{2}^{\{a, b+1\}}=\left[a \lambda_{1}+b \lambda_{2}\right]^{-1} \text { or } 0,
$$

the symplectic property comes without any extra effort. Thus, in our example if $\bar{\Lambda} \neq 0$ we take

$$
\nu=-\mu\left[a \lambda_{1}+b \lambda_{2}\right]^{-1} .
$$

It also follows that in the diagonal Hamiltonian case of dimension $2 N$ we only need $N$ nonconjugated components of the generating field.

In the application to periodic orbits in Sec. VI B, the full set of $2 N$ supplemented equations is not Hamiltonian but the original subset of $2 N-2$ equations is Hamiltonian. The above procedure also automatically preserves the Hamiltonian property within that subset if the variables (48b) are treated as parameters.

\section{Real transformation. Elliptic case}

The eigenvalues of $A$ are either pure real (hyperbolic, unstable central orbit) or pure imaginary (elliptic, stable central orbit). In the elliptic case $\lambda_{1,2}= \pm i \omega$, the "eigencoordinates" $z_{1,2}=q \pm i p$ are complex, and $\overline{z_{1}}=z_{2}$. This means that equations for $\dot{z}_{2}$ and $\dot{z}_{1}$ are complex conjugates, and therefore the corresponding components of all vector fields, $u, v, w, \tilde{u}$, etc., have the property $(u)_{2}=\overline{(u)_{1}}$ : the canonical conjugate is the complex conjugate [30].

Like the symplectic property, reality is preserved. In the example (57) above, if the original equations are real, the algorithm of the Lie transformation will always produce combinations such as

$$
w_{r}=2 i\left(\begin{array}{l}
\left(z_{1}^{a+1} z_{2}^{b}\right) /(a+1)+\left(z_{1}^{b+1} z_{2}^{a}\right) /(b+1) \\
-\left(z_{1}^{b} z_{2}^{a+1}\right) /(a+1)-\left(z_{1}^{a} z_{2}^{b+1}\right) /(b+1)
\end{array}\right)
$$

in the generator or the corresponding term

$$
W_{r}=2 i \frac{z_{1}^{a+1} z_{2}^{b+1}+z_{1}^{b+1} z_{2}^{a+1}}{(a+1)(b+1)}
$$

in the Hamilton function of the Lie generator (see Appendix E 1). Therefore, the transformed equations will automatically be real. The factor of $2 i$ appears in front of all vector fields [30]. [The two additional auxiliary variables (48b) also obey complex conjugation; we will see this in Eq. (62a)].

\section{Birkhoff terms}

The Hamiltonian case always possesses "uninteresting", "resonances.' In Eq. (56b) let $k=1,\left(m_{1}, m_{2}\right)=(2,1)$, and all other $m_{i}=0$; then

$$
(m, \lambda)-\lambda_{1}=2 \lambda_{1}+\lambda_{2}-\lambda_{1},
$$

and this always vanishes since $\lambda_{2}=-\lambda_{1}$. Similarly $k=2$ and $\left(m_{1}, m_{2}\right)=(1,2)$ also gives zero. More generally, these "resonances" occur when $m_{1}=m_{2} \pm 1$ for $k=1,2$, respectively [cf. Eq. (57d)] and it follows that

$$
\bar{\Lambda}_{1}^{\{a+1, a\}}=\bar{\Lambda}_{2}^{\{a, a+1\}}=0 \quad \text { for } r>0 .
$$


The corresponding null space terms that cannot be eliminated from the vector field (41), and its Hamilton function are

$$
\begin{gathered}
v_{r}=2 i\left(z_{1} z_{2}\right)^{a}\left(\begin{array}{c}
z_{1} \\
-z_{2}
\end{array}\right) \in \operatorname{Ker} \mathfrak{L}_{A}, \\
V_{r}=(2 I)^{a+1}(a+1)^{-1},
\end{gathered}
$$

with $r=2 a$ (cf. Sec. VI A 3). These resonances therefore produce a power series in $I$ in the normal form of the Hamilton function

$$
H_{\mathrm{NF}}=\omega I+\beta I^{2}+\cdots .
$$

In a time-independent one-degree-of-freedom Hamiltonian system with variables $(p, q)$ these are the only resonance terms, and they form a convergent Birkhoff series. The first term represents rotation in the $(p, q)$ plane, and the second is the shear associated with that rotation.

\section{Resonances " $z: \theta$ "}

Bifurcations occur when there is a resonance between the period of the central orbit and the period of the transverse vibrations (Sec. VI B). Therefore the resonances of primary interest to us occur between a transverse mode $\left(z_{1}, z_{2}\right)$ with eigenvalues $\lambda_{1,2}= \pm i \omega$ and the auxiliary system $\left(\zeta_{1}, \zeta_{2}\right)$, such as defined in (48b), with $\lambda_{3,4}= \pm i$. For example, suppose $\omega=n / k, n<k$, and $k>2$; then $k \lambda_{1}=n \lambda_{3}$. Physically we are saying that in $k$ periods of the original orbit the linearized equations give $n$ transverse oscillations, so we look for a period- $k$ bifurcation. In Eq. (56b) we consider $\{m\}=\left(m_{1}, m_{2}, m_{3}, m_{4}\right)=(0, k-1, n, 0)$, and we obtain

$$
(k-1) \lambda_{2}+n \lambda_{3}=\lambda_{1},
$$

which also implies

$$
(k-1) \lambda_{1}+n \lambda_{4}=\lambda_{2} .
$$

The null-space term in $(v)_{1}$ arising from (61a) is $z_{2}^{k-1} \zeta_{1}^{n}$. By the symplectic condition [or by Eq. (61b)], the term $-z_{1}^{k-1} \zeta_{2}^{n}$ must occur in $(v)_{2}$. The vector field and the associated term in the transformed Hamilton function are

$$
\begin{gathered}
v_{r}=2 i\left(z_{2}^{k-1} \zeta_{1}^{n},-z_{1}^{k-1} \zeta_{2}^{n}\right)^{T}, \\
V_{r}=2 i\left(z_{2}^{k} \zeta_{1}^{n}+z_{1}^{k} \zeta_{2}^{n}\right) k^{-1} \\
\rightarrow(2 I)^{k / 2}\left(e^{i[k(\varphi-\pi / 2)+n \theta]}+e^{-i[k(\varphi-\pi / 2)-n \theta]}\right) k^{-1} \\
=(2 I)^{k / 2} 2 \cos (k \varphi-k \pi / 2+n \theta) k^{-1},
\end{gathered}
$$

with $r=k-1+n$ (cf. Sec. VI A 3) and the polar representation as in Eq. (50a) [30]. This is the principal, lowest-order term of the $n: k$ resonance.

This same resonance produces many higher-order terms in the Hamilton function: on the left-hand side of Eq. (61a) we may add zero in the form $0=a\left(\lambda_{1}+\lambda_{2}\right)$ for any integer $a>0$ to obtain $\{m\}=(a, a+k-1, n, 0)$. This and its conjugate give

$$
\begin{aligned}
& a\left(\lambda_{1}+\lambda_{2}\right)+(k-1) \lambda_{2}+n \lambda_{3}=\lambda_{1}, \\
& a\left(\lambda_{1}+\lambda_{2}\right)+(k-1) \lambda_{1}+n \lambda_{4}=\lambda_{2}
\end{aligned}
$$

for $a \geqslant 0$. Furthermore, if $a>0$ we also need

$$
\begin{aligned}
& (a-1)\left(\lambda_{1}+\lambda_{2}\right)+(k+1) \lambda_{1}+n \lambda_{4}=\lambda_{3}, \\
& (a-1)\left(\lambda_{1}+\lambda_{2}\right)+(k+1) \lambda_{2}+n \lambda_{3}=\lambda_{2} .
\end{aligned}
$$

The resulting null-space terms are

$$
V_{r}=(2 I)^{a+k / 2} 2 \cos (k \varphi-k \pi / 2+n \theta) .
$$

Since all these resonance terms have the same angular dependence (62c), the effect of the resonance can be expressed more simply by means of a canonical transformation

$$
(I, \varphi)=\left(I, \tilde{\varphi}-\frac{n}{k} \theta+\frac{\pi}{2}\right)
$$

with generating function

$$
F_{2}(\widetilde{\varphi}, I)=I \widetilde{\varphi}-\frac{n}{k} I \theta+\frac{1}{2} I \pi
$$

This transformation expresses the resonant term (62c) as $V_{r}=(2 I)^{k / 2} 2 \cos (k \widetilde{\varphi}) / k$, and through $\partial F_{2} / \partial \theta$ it converts the linear term of the normal form $\omega I$ to $\alpha I=(\omega-n / k) I$, which vanishes at the resonance. Hence we obtain a "timeindependent" formula for the normal form

$$
\begin{gathered}
H_{\mathrm{NF}}=\alpha I+\beta I^{2}+\cdots+\gamma I^{k / 2} \cos (k \widetilde{\varphi})+\cdots, \\
\alpha=\left(\omega-\frac{n}{k}\right) \sim 0 .
\end{gathered}
$$

Higher-order resonant terms are $V_{r}=I^{k / 2+a} \cos (k \widetilde{\varphi})$, with integer $a>0$ and $r=2 a+k-1+n$. [Recall the comment above Eq. (5): $\cos k \widetilde{\varphi}$ is combined with $I^{k / 2}$ and higher.] Of course, the normal form combines these resonant terms (64a) with Birkhoff terms (59d).

The time dependence has been moved out of the Hamilton functions and into the coordinates, so that the old coordinate and momentum in the transverse plane are

$$
y=\left(\begin{array}{c}
\sigma \\
p_{\sigma}
\end{array}\right)=\sqrt{2 I}\left(\begin{array}{c}
\sin (\widetilde{\varphi}+\pi / 2-n \theta / k) \\
\cos (\widetilde{\varphi}+\pi / 2-n \theta / k)
\end{array}\right) .
$$

In particular at $\theta=0(\sqrt{2 I}, \widetilde{\varphi})$ are standard polar coordinates for the frame $y=\left(\sigma, p_{\sigma}\right)$. Thus the contour plot of $H_{\mathrm{NF}}(\sqrt{2 I}, \widetilde{\varphi})$ in $(66)$ corresponds to the Poincare section at $\theta=0$. More generally, all Poincaré sections transverse to the orbit have now been made to correspond to contour plots of a function of two variables.

\section{Generic structure of bifurcations and sequences of bifurcations}

Equations (66) bring us back to Meyer's theorem on the generic structure of bifurcations of periodic orbits. Stationary points of a $C_{k}$-symmetric function of two variables (66) correspond to periodic orbits in a period- $k$ bifurcation (Sec. II B 3), so that the creation of new orbits is made visible in contour plots of (66). We find all generic structures by con- 
sidering all possible values of $k>2$ and all possible values of the parameters $\alpha, \beta$, and $\gamma$, remembering that $\alpha$ is small and passes through zero at the bifurcation (cf. Table I). This normal form (66) does not describe cases $k=1$ and $k=2$, since there the resonance occurs within the linear problem (Sec. VIII).

The quantitative implementation of the procedure to obtain (66) gives much more. By extending the normal form to higher-order terms, evaluating (numerically) all the relevant coefficients, we learn not only what can happen in general, but what does happen in any particular system. Thus, the normal form may predict the regular sequences of bifurcations and the order in which the bifurcations occur as the parameter(s) vary (Secs. I and II A 1). In the following section we will carry out the procedure for the perpendicular orbit, and thereby explain the sequences of bifurcations that exist in this system.

\section{B. Normal forms near period- $k, k>2$, resonances of the perpendicular orbit}

As we have already discussed in Secs. IV C 3 and V D 2, special symmetry properties of the perpendicular orbit $R_{\lambda}$ and $\mathrm{R}_{\sigma}$ result in some "nongeneric" features of the reduced problem, and hence of the normal forms that are obtained in this section. In a sense, however, these symmetries make things simpler and more suited to highlight major aspects of the general theory.

To obtain normal forms of one-degree-of-freedom $2 \pi$-periodic Hamilton equations $\dot{x}=A_{\varepsilon} x+v_{\varepsilon}(x, \widetilde{\theta})$ resulting from the Floquet transformation in Sec. V D we introduce auxiliary variables (48b) and reduce the system of four (nonlinear) differential equations following the method in Sec. VII A.

We begin by replacing polar coordinates $(I, \varphi)$ and time $\widetilde{\theta}$ in Eqs. (35) and (36b) by complex coordinates $\left(z_{1}, z_{2}\right)$ and auxiliary variables $\left(\zeta_{1}, \zeta_{2}\right)$ in Eqs. (48b). (Note that in the definition of $\zeta$ we use $\widetilde{\theta}=2 \theta$ introduced in Sec. V D 2.) The Hamilton function (36b) becomes

$$
\mathcal{J}_{\varepsilon}(z, \zeta)=i \omega z_{1} z_{2}+i \sum_{\substack{s=0 \\ b=0}}\left(z_{1} z_{2}\right)^{s} \sum_{\substack{a=0 \\ 2 a+s \geqslant 4}}\left(f_{\varepsilon, s}^{(a, b)}+g_{\varepsilon, s}^{(a, b)}\right),
$$

where $f_{\varepsilon, s}^{(a, b)}$ and $g_{\varepsilon, s}^{(a, b)}$ are coefficients derived from Eq. (36b) times functions $f^{(a, b)}$ and $g^{(a, b)}$ defined below:

\begin{tabular}{llll}
$a$ & $b$ & $f^{(a, b)}(z, \zeta)$ & $g^{(a, b)}(z, \zeta)$ \\
\hline$a=0$ & $b=0$ & 1 & \\
$a>0$ & $b=0$ & $z_{1}^{2 a}+z_{2}^{2 a}$ & \\
$a=0$ & $b>0$ & $\zeta_{1}^{b}+\zeta_{2}^{b}$ & \\
$a>0$ & $b>0$ & $z_{1}^{2 a} \zeta_{1}^{b}+z_{2}^{2 a} \zeta_{2}^{b}$ & $z_{1}^{2 a} \zeta_{2}^{b}+z_{2}^{2 a} \zeta_{1}^{b}$
\end{tabular}

Due to the $\mathrm{R}_{\sigma}$ symmetry of the orbit, the powers of $z$ are even. [(36b) is $\pi$ periodic in $\varphi$.] The Hamilton function (68) without the overall factor $2 i$ [30] is real, and moreover, all coefficients $f_{\varepsilon, s}^{(a, b)}$ and $g_{\varepsilon, s}^{(a, b)}$ are real.

The Lie transformation of the vector field of (68) naturally preserves these properties. The $a=b=0$ terms are the
TABLE IV. Normal forms near $1: k$ bifurcations of the perpendicular orbit.

\begin{tabular}{lcccc}
\hline \hline & $f^{\text {a }}$ & $f^{(0)}$ & $f^{(1)}$ & $f^{(2)}$ \\
\hline \multicolumn{4}{c}{$1: 3$ resonance at $\varepsilon_{\text {crit }}=-0.4830( \pm 0.0028)^{\mathrm{b}}$} \\
$I$ & $\alpha$ & & -0.797772 & -1.98580 \\
$I^{2}$ & $\beta_{1}$ & 0.0432953 & -0.499818 & -2.76776 \\
$I^{3}$ & $\beta_{2}$ & 0.351321 & 1.12411 & 4.70299 \\
& $\gamma_{2}$ & 0.00473866 & 0.0414095 & 0.595889 \\
$I^{4}$ & $\beta_{3}$ & 0.415886 & 2.51807 & 25.8660 \\
$I^{5}$ & $\beta_{4}$ & -0.357956 & -2.38341 & -14.1571 \\
& & $1: 4$ resonance at $\varepsilon_{\text {crit }}=-0.3160$ & \\
$I^{2}$ & $\alpha$ & & -1.25598 & -4.05203 \\
$I^{2}$ & $\beta_{1}$ & -0.108838 & -1.63900 & -15.9104 \\
& $\gamma_{1}$ & 0.0440067 & 0.265658 & 2.77952 \\
$I^{3}$ & $\beta_{2}$ & 0.710901 & 3.93034 & 36.2895 \\
& $\gamma_{2}$ & 0.194832 & 1.72993 & 25.6648 \\
$I^{4}$ & $\beta_{3}$ & 1.92713 & 26.4671 & 521.614 \\
& $\gamma_{3}$ & 0.342389 & 4.39222 & 83.3024 \\
\hline \hline
\end{tabular}

${ }^{\mathrm{a} C o e f f i c i e n t s} f=\alpha, \beta, \gamma$ in Eq. (69b) are approximated as $f(\varepsilon)=f^{(0)}+f^{(1)}\left(\varepsilon-\varepsilon_{\text {crit }}\right)+1 / 2 f^{(2)}\left(\varepsilon-\varepsilon_{\text {crit }}\right)^{2}$.

${ }^{\mathrm{b}}$ Range of $\varepsilon-\varepsilon_{\text {crit }}$ used in our study.

always present "uninteresting" resonances (Birkhoff terms in Sec. VII A 4). When $\omega \sim n / k$ some of the terms $g^{(a, b)}(z, \zeta)$ become (nearly) resonant terms of type " $z: \theta$." From Eq. (62b) we conclude that the lowest resonance term is such that $(a, b)$ are

$$
(2 a, b)_{\text {res }}= \begin{cases}(k, n) & \text { for even } k \\ (2 k, 2 n) & \text { for odd } k .\end{cases}
$$

Then if we define $\kappa=2 a_{\text {res }}$, the normal form is

$$
H_{n: k}=\alpha(\varepsilon) I+\sum_{s=1} \beta_{s}(\varepsilon) I^{s+1}+\sum_{s=\kappa / 2-1} \gamma_{s}(\varepsilon) I^{s+1} \cos (\kappa \varphi) .
$$

To find how coefficients $\alpha, \beta_{s}$, and $\gamma_{s}$ depend on $\varepsilon$ we obtain $H_{n: k}$ at different values $\varepsilon \sim \varepsilon_{\text {crit }}^{n: k}$ and approximate the coefficients by a power series. (Usually a parabolic threepoint fit suffices.) Of course,

$$
\alpha\left(\varepsilon_{\text {crit }}^{n: k}\right) \equiv 0 .
$$

Note that we have thereby explicitly derived the rules found by Mao and Delos for bifurcations of the perpendicular orbit ([6], Sec. III B); in particular, Eq. (69a) explains why only odd- $k$ bifurcations differ from the generic $k$ bifurcation of Meyer and form " $2 k$-island chain" structures. [Thus for the period-3 bifurcation $k=3$ in Eq. (69a), so $\kappa=6$; cf. Ref. [12].] Furthermore, as we show below, the $I$ dependence of the principal terms $\beta$ and $\gamma$ explains other bifurcations occurring near the central orbit.

Coefficients in (69b) that we obtained for the perpendicular orbit are given in Table IV. A particular result, which is not related to symmetry, is that the period-4 bifurcation has the "island-chain" structure $\left(\left|\beta_{1}\right|>\left|\gamma_{1}\right|\right)$ rather than the "touch-and-go", structure (cf. Table I). 
Periodic trajectories correspond to the stationary points of normal form (69b). These points are easy to find because they must lie on radial lines with angles $\varphi_{0}$ such that

$$
\left\{\varphi_{0}: \frac{\partial H_{n: k}}{\partial \varphi}\left(\varphi_{0}\right)=0\right\}=\left\{0, \frac{\pi}{\kappa}, \frac{2 \pi}{\kappa}, \ldots\right\} .
$$

All we need is to consider two sections of (69b) - for $\varphi=0$ and $\pi / \kappa$, and to find stationary points in these sections. The stability of the points (and of the orbits) can be easily determined by comparing the sections. For instance, a point $\left(I_{0}, \pi / \kappa\right)$ is a saddle if it is a minimum in the $\varphi=\pi / \kappa \mathrm{sec}-$ tion but is higher than $\left(I_{0}, 0\right)$.

\section{Symmetries and configuration-space images}

Further analysis of the relation between the stationary points or, more generally, the contour plot of (69b) and the actual periodic trajectories involved requires reexamination of the symmetries $\mathrm{R}_{\sigma}$ and $\mathrm{R}_{\lambda}$, that were considered briefly in Sec. IV C 3. The Hamilton function and the perpendicular orbit are invariant under these order-2 operations.

As is well known, at a bifurcation stable and unstable orbits are created simultaneously. In our case the new orbits cannot retain all the order- 2 symmetries of their parent. They must break a symmetry, and therefore they must appear as twins connected by the operation of the broken symmetry.

The normal form near the $k$ bifurcation has a number of stable and unstable stationary points, which correspond to fixed points of the $k$ th iterate of the Poincare half map $\left[\lambda=0, p_{\lambda}\right.$ either sign $]$. The half map itself connects these points in sets of $k$ points, and thus defines the correspondence between the stationary points of the normal form and the periodic trajectories.

Detailed analysis shows that the following is true at every $1: k$ bifurcation of the perpendicular orbit with $k \geqslant 3$ : (1) Four distinct phase-space periodic orbits are either created or destroyed. Two are stable and two are unstable. (2) At each $k$ every new orbit is invariant under one of the two symmetry operations, $R_{\sigma}$ or $R_{\lambda}$ (i.e., they are all invariant under $\mathrm{R}_{\sigma}$ or they are all invariant under $R_{\lambda}$ ). The other operation converts one twin orbit into another (i.e., the other symmetry is broken by the bifurcation). (3) If $k$ is odd, $\mathrm{R}_{\sigma}$ is broken; each of the four new orbits is transformed into itself under $R_{\lambda}$. The new orbits are labeled $\Gamma_{i}$, and they are related by

$$
\begin{array}{cc}
R_{\sigma} & R_{\sigma} \\
\Gamma_{0} \leftrightarrow \Gamma_{2}, & \Gamma_{1} \leftrightarrow \Gamma_{3}
\end{array} \text { for odd } k .
$$

If $k$ is even, $\mathbf{R}_{\lambda}$ is broken and each new orbit is transformed into itself under $\mathrm{R}_{\sigma}$. The twins are called $\Gamma_{i}^{ \pm}$, and

$$
\Gamma_{0}^{+} \stackrel{R_{\lambda}}{\leftrightarrow} \Gamma_{0}^{-}, \quad \Gamma_{1}^{+} \stackrel{R_{\lambda}}{\leftrightarrow} \Gamma_{1}^{-} \quad \text { for even } k .
$$

Of course, twin orbits have the same stability and other properties [43].

(4) If $k$ is odd the Poincare half map $\left[\lambda=0, p_{\lambda}\right.$ either sign] shows $k+k$ stable $\mathrm{O}$ points and $k+k$ unstable $\mathrm{X}$ points. Each such set of $k$ points corresponds to a periodic trajectory with period $2 k$ times the half-map period, i.e., $k$ times the full period of the perpendicular orbit in $\left(u^{\prime}, v^{\prime}\right)$

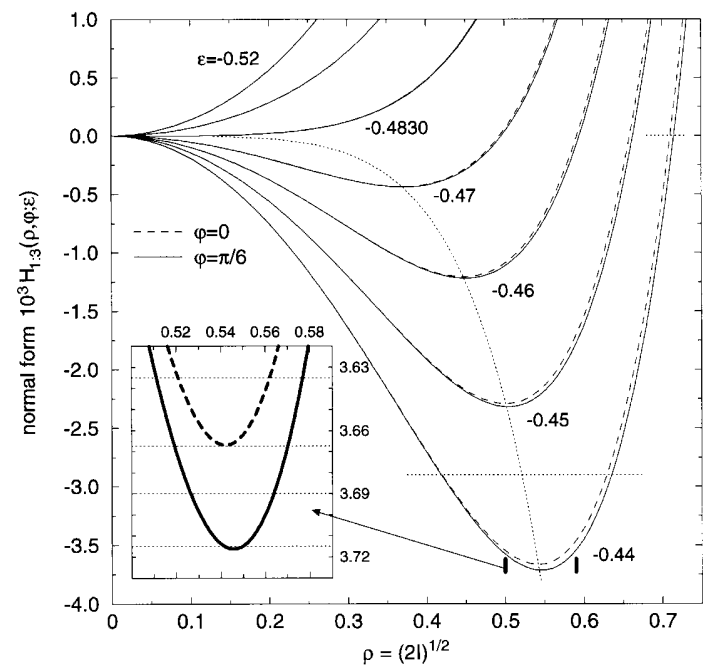

FIG. 12. Normal form near the 1:3 bifurcation of perpendicular orbit [cf. Table IV and Eq. (69b)] at various scaled energies $\varepsilon$. Dotted lines give the position of constant level sections in Fig. 13.

space. In a full period of the new orbit an $\mathrm{O}$ point (or an $\mathrm{X}$ point) is visited twice, once with $p_{\lambda}>0$ and once with $p_{\lambda}<0$.

(5) If $k$ is even the half map shows $k \mathrm{O}$ points and $k \mathrm{X}$ points. Each set of $k$ points corresponds to two periodic trajectories, one with $p_{\lambda}>0$ and one with $p_{\lambda}<0$. The period of those orbits is $k$ times the half-map period, i.e., $k / 2$ times the full period of the perpendicular orbit in $\left(u^{\prime}, v^{\prime}\right)$ space.

(6) In configuration space $\left(u^{\prime}, v^{\prime}\right)$ one pair of twin orbits forms loops and the other forms lines (see Figs. 13 and 15). Loops are $\Gamma_{1}, \Gamma_{3}$ and $\Gamma_{0}^{+}, \Gamma_{0}^{-}$; lines are $\Gamma_{0}, \Gamma_{2}$ and $\Gamma_{1}^{+}, \Gamma_{1}^{-}$.

(7) One pair of twins passes through the origin $u^{\prime}=v^{\prime}=0 \quad(\sigma=\lambda=0) \quad$ and the other does not. If $k \bmod 4=2$ (i.e., if $k$ is even but $k / 2$ is odd) then the lines pass through the origin. Otherwise it is the loops that pass through the origin.

All this is proven in Appendix F.

\section{Bifurcation 1:3}

Table IV gives coefficients in the normal form for the 1:3 bifurcation and Figs. 12 and 13 give plots of this normal form. SMD ([11,6], Fig. 8) computed surfaces of section near this bifurcation. The contour plot of our normal form (Fig. 13) is indistinguishable from their numerically generated SOS. The 1:3 case is unusual among the bifurcations of the perpendicular orbit. Most of the other bifurcations follow the organized sequence discussed in Sec. I (Fig. 1). However, in the 1:3 case, $\beta_{1}$ and $\beta_{2}$ have the same sign, and the organized sequence is not present. Instead a single symmetric period-3 bifurcation takes place and new PO's are created directly at the central orbit. Additional details of this case follow.

The case $\varepsilon=E B^{-2 / 3} \rightarrow-\infty$ corresponds to the zero field $(B \rightarrow 0)$ bound $(E<0)$ Coulomb problem and is integrable. Therefore, according to the KAM theorem most of the invariant tori of the Coulomb problem are preserved if $\varepsilon$ is sufficiently low. The 1:3 bifurcation of the perpendicular orbit occurs at such low $\varepsilon$ (cf. Fig. 9). Thus, as we see in Table 

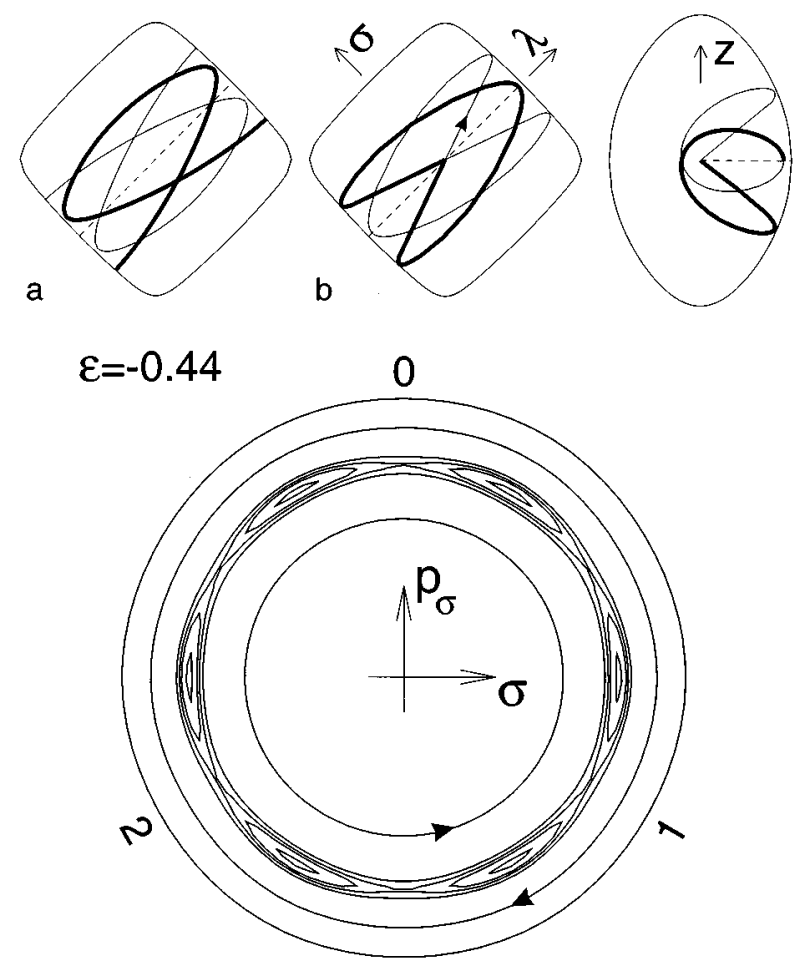

FIG. 13. Periodic trajectories created in the period-3 bifurcation (above): (a) stable trajectories $\Gamma_{0,2}$ in $(u, v)$ coordinates; (b) unstable trajectories $\Gamma_{1,3}$ in $(u, v)$ and $(\rho, z)$ coordinates. Constant level sections of the 1:3 normal form (below).

IV the resonance term $\gamma I^{3} \cos (\pi / 3)$, which, in a sense, reflects nonintegrability, is very small compared to Birkhoff terms $(|\gamma / \beta| \ll 1)$, so that $H_{1: 3}$ has only a small dependence on angle $\varphi$. This is well demonstrated in Fig. 12 where the entire angular variation of the reduced Hamilton function $H_{1: 3}$ is confined between the solid and the dashed lines, and is $\sim 1 \%$ of the radial variation.

Most (all at $\varepsilon<\varepsilon_{\text {crit }}$ ) of the constant level sets of $H_{1: 3}$ are (concentric) circles and represent the invariant tori of the perturbed Coulomb problem. In other words, the action $I$ is not only well preserved locally (in the neighborhood of the perpendicular orbit) but in fact it is an approximate integral of motion for the whole problem. Each invariant torus is densely filled by quasiperiodic trajectories that coil around the perpendicular orbit. The speed of the coil, and in particular its direction (the direction of the Poincare map), relative to $\omega=1 / 3$, the speed of our reference frame [cf. Eq. (65)], is given by $\dot{\varphi}=\partial H_{1: 3} / \partial I$. At $\varepsilon<\varepsilon_{\text {crit }}$ this $\dot{\varphi}(I)>0$, whereas at $\varepsilon>\varepsilon_{\text {crit }} \dot{\varphi}(I)$ is negative if $I<I_{0}$ and positive if $I>I_{0}$. In other words, as shown by arrows in Fig. 13 trajectories coil slower than $2 \pi / 3$ per period near the central orbit, and faster than $2 \pi / 3$ if far from the center. $I=I_{0}$ corresponds to a resonant torus that is destroyed by the bifurcation. The position $I_{0}$ corresponds to $\dot{\varphi}=0$, the minimum of the curves in Fig. 12 . Thus $I_{0}(-0.44)=0.147(\rho=0.542)$.

The destroyed resonant torus and its narrow vicinity where the original Hamiltonian could exhibit chaotic dynamics can be well observed in the contour plot in Fig. 13. The new periodic orbits are the "bones" left from this torus (to visualize the torus, superimpose the two upper left plots in Fig. 13). To find the correspondence between the contour

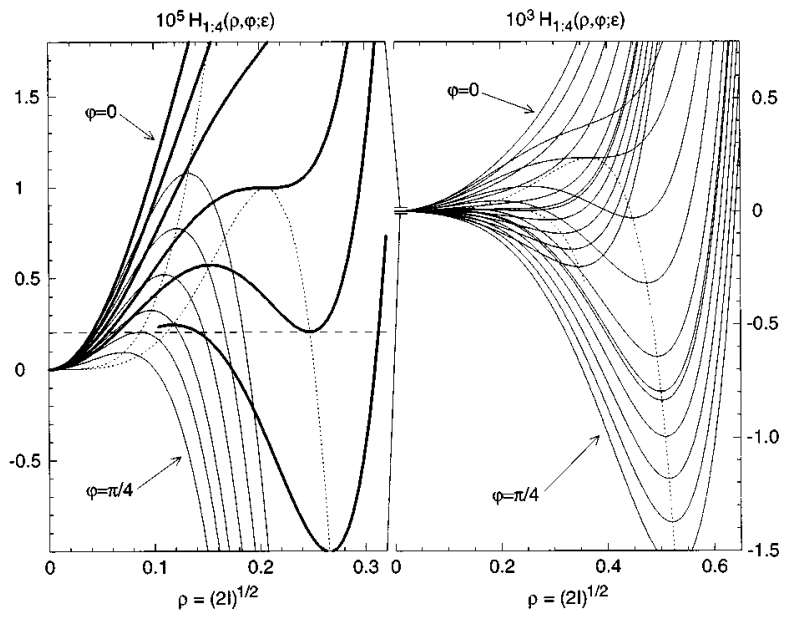

FIG. 14. Normal form near the 1:4 bifurcation of the perpendicular orbit [cf. Table IV and Eq. (69b)] at various scaled energies $\varepsilon$. Dotted lines show the position of stationary points; the dashed line on the enhanced graph (left) shows the position of the unified separatrix [cf. Fig. 1(c)]. Sections are plotted for $10^{3}\left(\varepsilon_{1: 4}-\varepsilon\right)=10$ (top trace in each family), 8.12, 6, 4, 2, 1.1, $0.88,0,-1,-2$, and -3 on the right; and $2,1.7,1.4,1.1,0.88$, 0.6 on the left.

plot in Fig. 13, the half-period Poincare map, and the actual periodic trajectories refer to the general outline in Sec. VII B 1. For instance, the orbit whose direction is shown in Fig. 13(a) by an arrow, starts from the origin and passes (twice) the points 0,1 , and 2 . Hence this is $\Gamma_{3}$. It and its twin $\Gamma_{1}$ are unstable.

\section{Bifurcation 1:4}

The period-4 bifurcation, even though it follows next to period 3 at $\varepsilon_{1: 4}=-0.31627$ (Fig. 9), occurs in a very different domain. The global near integrability has been destroyed, and a large part of phase space is chaotic. The absence of integrability is well demonstrated by the profound difference of the sections at $\pi / 4$ (deep minimum) and 0 shown in Fig. 14. The corresponding contour plots are shown in Fig. 1.

The most important qualitative difference in the 1:4 normal form is that the major nonlinear terms alternate sign: in Table IV $\beta_{1} \pm \gamma_{1}<0$ but $\beta_{2} \pm \gamma_{2}>0$. This causes a more complicated phenomenon, the organized sequence of three bifurcations, already discussed in Secs. I and II A 1.

We consider function $H_{1: 4}$ in Table IV and easily find that the sequence begins with the saddle-node $\left(C_{1}\right)$ bifurcation at $\varphi=\pi / 4$ and $\varepsilon^{\prime}=\varepsilon_{1: 4}-0.008123=-0.324403$ followed by a similar bifurcation at $\varphi=0$ and $\varepsilon^{\prime \prime}=\varepsilon_{1: 4}-0.001106=-0.317386$. Shortly after that, at $\varepsilon_{1: 4}-0.000882=-0.317162$ the two saddle points change their relative position so that a qualitative change of the separatrices occurs. [The moment when the two saddles are at the same level is shown by the dashed line in Fig. 14; it corresponds to a single "degenerated" separatrix nearly shown in Fig. 1(c).] All these values completely agree with those observed numerically by Mao and Delos ([6], Sec. V C 3) (cf. their $\varepsilon^{\prime} \approx-0.325$, and $\varepsilon^{\prime \prime} \approx-0.3173$ ).

Eight periodic orbits are involved; the ones that eventually survive are shown in Fig. 15. The other four are similar 


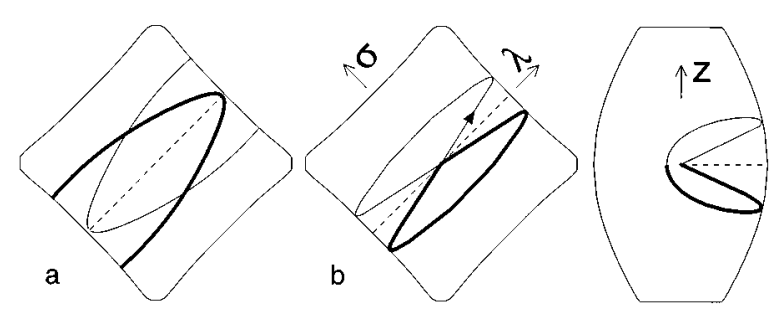

FIG. 15. Periodic trajectories created near the perpendicular orbit before it undergoes a half period-4 (actual period-2) bifurcation: (a) stable trajectories in $(u, v)$ coordinates $\Gamma_{1}^{ \pm}$; (b) unstable trajectories $\Gamma_{0}^{ \pm}$or "pacmen" in $(u, v)$ and in $(\rho, z)$ coordinates for scaled energy $\varepsilon=-0.31$.

to those shown, they contract to the perpendicular orbit and disappear at $\varepsilon_{1: 4}$.

\section{Bifurcations at higher $\varepsilon$}

The alternation of signs in the normal form and the concomitant organized sequences of bifurcations of perpendicular PO persist at higher $\varepsilon$, where the other $1: k$ bifurcations occur (Fig. 9). Except for the 1:3 bifurcation at low $\varepsilon$ (Sec. VII B 2 and Fig. 13), all of the $1: k$ bifurcations that we and SMD $[6,11]$ examined exhibit the same organized sequence.

This raises an interesting question. The last bifurcation of the perpendicular PO occurs when it goes unstable at $\varepsilon=-0.1273$. Calculations $[6,11]$ had shown that at this point two unstable PO's collide with the perpendicular PO and disappear, so it is a pitchfork bifurcation (Table I, $C_{2}$ type, case $a b<0$ ). Does this bifurcation also occur through an organized sequence? If so, we would look for a twin saddle-node bifurcation creating twin stable-unstable pairs of PO's at some lower $\varepsilon$ with the two twin unstable PO's closer to the perpendicular orbit in the center; then as $\varepsilon$ increases we would expect these unstable PO's move towards the center and disappear.

To answer this question, we need to create some more machinery, because for this $C_{2}$ bifurcation, the matrix $\Omega\left(\varepsilon_{\text {crit }}\right)$ is singular, and cannot be put into diagonal form. The Lie-transformation scheme is given in the following section.

\section{NORMAL FORM IN THE SINGULAR CASE}

The above construction in Sec. VII A presumed that the matrix $A$ representing the linear part of the differential equations and the associated matrix representation of $\mathfrak{L}_{A}$ could be put into diagonal form. The procedure, and the resulting normal form, describes all $k$ bifurcations with $k \geqslant 3$. The $k=1$ and $k=2$ resonances are distinct cases. The matrix $A$, and the associated representation of $\mathfrak{L}_{A}$, cannot be converted to diagonal form. Therefore a different approach is required.

The essential result is the following ([17], Chap. 6, Sec. 35D): given

$$
\left(\begin{array}{l}
\dot{x}_{1} \\
\dot{x}_{2}
\end{array}\right)=\left(\begin{array}{ll}
0 & 1 \\
0 & 0
\end{array}\right)\left(\begin{array}{l}
x_{1} \\
x_{2}
\end{array}\right)+u\left(x_{1}, x_{2}\right)
$$

normalization converts $u(x)$ to

$$
v\left(x_{1}, x_{2}\right)=\left(\begin{array}{c}
0 \\
a x_{1}^{2}+b x_{1} x_{2}+\ldots
\end{array}\right) .
$$

In the Hamiltonian case $b x_{1} x_{2}$ is forced to be zero. That leads to a Hamilton function in the form

$$
H_{\mathrm{NF}}\left(x_{1}, x_{2}\right)=\frac{1}{2} x_{2}^{2}-\frac{1}{3} a x_{1}^{3}+\cdots
$$

The lowest terms in the normal form of the Hamilton function represent a particle moving on a cubic potential curve [cf. Eq. (2a)]. For period 2 or in the case of symmetry $C_{2}$, the normal form of the Hamilton function is

$$
H_{\mathrm{NF}}\left(x_{1}, x_{2}\right)=\frac{1}{2} x_{2}^{2}-\frac{1}{4} a x_{1}^{4}+\cdots
$$

[cf. Eq. (2b)], a quartic potential energy.

As before, to get a quantitative scheme for generating higher-order terms, we have to construct carefully a rather elaborate procedure.

\section{A. General procedure}

Matrices $A$ in a one-parameter generic family have at most one $2 \times 2$ Jordan block (71a) at an isolated value of the parameter ([17], Chap. 6, Sec. 30E, Corollary on p. 246). In the Hamiltonian case the diagonal elements of this block vanish [24].

Consider a problem of dimension $N$ with a singular linear part, and let (symplectic) coordinates $\left(x_{1}, x_{2}\right)$ span the singular subspace of $A$ in (38) and $\left(z_{3}, z_{4}, \ldots, z_{N}\right)$, the regular subspace where $A$ has been brought to diagonal form $\operatorname{diag}\left(\lambda_{3}, \lambda_{4}, \ldots\right)$, so that

$$
\left(\begin{array}{c}
\dot{x} \\
\dot{z}
\end{array}\right)=A\left(\begin{array}{l}
x \\
z
\end{array}\right)+u(x, z)=\left(\begin{array}{c}
x_{2} \\
0 \\
\lambda_{3} z_{3} \\
\vdots
\end{array}\right)+u(x, z) .
$$

More generally, we study a parametric family of matrices $A$ near a singular point, such as

$$
A=\left(\begin{array}{cc|ccc}
0 & 1 & & & \\
\alpha & 0 & & & \\
\hline 0 & & \lambda_{4} & \\
& & \lambda_{3} & & \\
0 & & & \ddots
\end{array}\right), \quad \alpha \sim 0,
$$

with off-diagonal element $\alpha$ and eigenvalues $\lambda$ depending on the parameter(s) of our problem $\varepsilon$ (not to be confused with $\epsilon$, the formal parameter of Lie theory). At the critical point $\alpha\left(\varepsilon_{\text {crit }}\right)=0$ and $A$ in (72b) is singular-a $C_{1}$ bifurcation occurs.

The idea of the transformation is basically the same: we introduce vector monomials, similar to those in Eqs. (47a) and (53), and try to solve the Lie equation (43c), i.e., to "remove all removable terms" from $u(x)$. Having the recipe for Eq. (43c) we apply iteration (44d). The problem is that we no longer work with an eigenbasis of $\mathfrak{L}_{A}$. The key to the solution is that $\operatorname{Ker}_{\mathfrak{L}_{A}}$ can still be constructed in terms of (53). 


\section{Vector valued polynomial basis}

The major property of operator $\mathfrak{L}_{A}$, already used in Secs. VI A 3 and VII A, is that it conserves the total power in $(x, z)$. It, in fact, separately conserves total powers in $x$ and in $z$, and furthermore it is clear that for $z_{3}, \ldots, z_{N}$, the coordinates on the regular subspace, $\mathfrak{L}_{A}$ conserves each individual power $m_{3}, \ldots, m_{N}$. This approves the construction of the following vector valued functions:

$$
w^{\{l, m\}}(x, z)=w^{\{l\}}(x) z^{\{m\}}=\left(\begin{array}{c}
w_{1}(x) \\
w_{2}(x) \\
w_{3}(x) \\
\vdots
\end{array}\right) z_{3}^{m_{3}} \cdots z_{N}^{m_{N}} .
$$

Here the functions $w_{j}(x)$ are homogeneous polynomials in $\left(x_{1}, x_{2}\right)$ of fixed degree $l$, and $z^{\{m\}}$ is a monomial in $z_{3}, \ldots, z_{N}$, with fixed individual powers [cf. Eqs. (47b) and (47c)]. The derivative [cf. Eq. (43b)] is given by

$$
\frac{d w(x, z)}{d(x, z)}=\left(\begin{array}{cc|ccc}
\frac{\partial w_{1}}{\partial x_{1}} & \frac{\partial w_{1}}{\partial x_{2}} & \frac{m_{3} w_{1}}{z_{3}} & \frac{m_{4} w_{1}}{z_{4}} & \ldots \\
\frac{\partial w_{2}}{\partial x_{1}} & \frac{\partial w_{2}}{\partial x_{2}} & \frac{m_{3} w_{2}}{z_{3}} & \frac{m_{4} w_{2}}{z_{4}} & \ldots \\
\hline \vdots & \vdots & \vdots & \vdots & \ddots
\end{array}\right) z^{\{m\}}
$$

and one can show that subspaces spanned by vectors (73a) (together with 0) with fixed $l$ and $\{m\}$ (and hence $l+m$ ) are indeed invariant under the action of $\mathfrak{L}_{A}$. Henceforth we work in each subspace of polynomials with fixed degree $l$ and fixed set $\{m\}$.

\section{Solving the Lie equation for $\alpha=0$}

Let $w$ and $u$ be vector-valued polynomials of type (73a). We first consider a pure singular case $\alpha=0$, such that

$$
\begin{aligned}
\mathfrak{L}_{A} w & =\left(\frac{d w(x, z)}{d(x, z)}\right)\left(\begin{array}{c}
x_{2} \\
0 \\
\lambda_{3} z_{3} \\
\vdots
\end{array}\right)-\left(\begin{array}{c}
w_{2} \\
0 \\
\lambda_{3} w_{3} \\
\vdots
\end{array}\right) \\
& =\left(\begin{array}{c}
\mathfrak{P} w_{1}-w_{2} \\
\mathfrak{P} w_{2} \\
\ldots \\
\left(\mathfrak{P}-\lambda_{k}\right) w_{k} \\
\ldots
\end{array}\right) z^{\{m\}}=\left(\begin{array}{c}
u_{1} \\
u_{2} \\
\ldots \\
u_{k} \\
\ldots
\end{array}\right) z^{\{m\}},
\end{aligned}
$$

$$
\mathfrak{P}=(\lambda, m)+x_{2} \frac{\partial}{\partial x_{1}} .
$$

We can always solve the first row in Eq. (74b) to obtain

$$
w_{2}(x)=\mathfrak{P} w_{1}(x)-u_{1}(x) \text {. }
$$

In other words, we can always find $w_{2}(x)$ such that in the normal form $u_{1} \rightarrow v_{1}=0$.

Evaluation of $v_{2}$ is more complicated. Substitution of (75a) into the second row of Eq. (74b) gives an equation for $w_{1}$, while the rest remains the same as in Eq. (74b):

$$
\mathfrak{P}^{2} w_{1} \stackrel{?}{=} u_{2}+\mathfrak{P} u_{1},
$$

$$
\left(\mathfrak{P}-\lambda_{k}\right) w_{k}=\stackrel{?}{=} u_{k} \quad \text { for } k>2 .
$$

For these equations to be solvable with arbitrary right-hand side (rhs) the kernel of $\mathfrak{P}$ and $\mathfrak{P}-\lambda_{k}$ should be empty. This occurs only if $(\lambda, m) \neq 0$ and $(\lambda, m)-\lambda_{k} \neq 0$.

The convenient representation of $w_{j}(x)$ and $\mathfrak{P}$ is the set of monomials of degree $l, x_{1}^{l_{1}} x_{2}^{l-l_{1}}$, which span an $(l+1)$-dimensional invariant subspace of the operator $\mathfrak{P}$. In this subspace we find from $(74 c)$ that $\mathfrak{P}$ is represented by the matrix

$$
\begin{aligned}
& {[l] \quad[l-1] \cdots \quad[2][1] \mid[0] \quad w / u}
\end{aligned}
$$

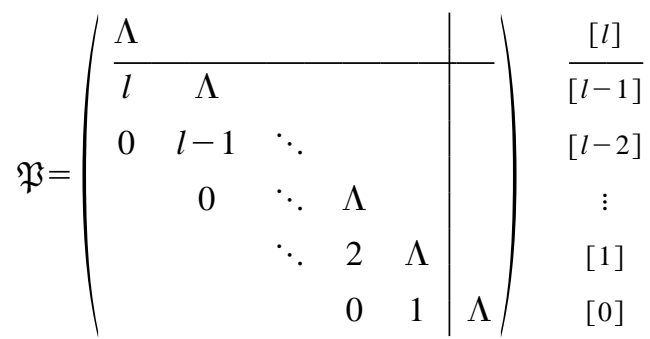

Rows and columns are labeled by the value of $l_{1}$, and $\Lambda=(\lambda, m) . \mathfrak{P}-\lambda_{k}$ in Eq. (75c) is represented by a matrix of the same form, but with $\Lambda=(\lambda, m)-\lambda_{k}$. The matrix (76) has rank $l+1$ only if $\Lambda \neq 0$. If $(\lambda, m) \neq 0$ or $(\lambda, m)-\lambda_{k} \neq 0$ then Eq. (75b) or (75c) has a unique solution $w$, which eliminates the corresponding terms $u(x, z)$ from the normal form.

Referring to Eq. $(75 \mathrm{c})$, if $\Lambda=(\lambda, m)-\lambda_{k}=0$, the $x_{1}^{l}$ component of $u_{k}(x)$ cannot be eliminated, and the $x_{2}^{l}$ component of $w_{k}(x)$ is undetermined (KerP $=x_{2}^{l}$ ). Turning to Eq. $(75 b)$, the matrix of $\mathfrak{P}^{2}$ is lower tridiagonal:

$$
\begin{gathered}
\left(\mathfrak{P}^{2}\right)^{i}{ }_{i}=\Lambda^{2}, \quad i=0, \ldots, l \\
\left(\mathfrak{P}^{2}\right)^{i}{ }_{i+1}=2 \Lambda(l-i), \quad i=0, \ldots, l-1,
\end{gathered}
$$

$$
\left(\mathfrak{P}^{2}\right)^{i}{ }_{i+2}=(l-i)(l-i-1), \quad i=0, \ldots, l-2 .
$$


When $\Lambda=(\lambda, m)=0$ this matrix has rank $l-2$, and $\operatorname{KerP}^{2}=\left\{x_{1} x_{2}^{l-1}, x_{2}^{l}\right\}$ represents the undetermined terms in $w_{1}(x)$. The terms that we cannot eliminate from the rhs of Eq. (75b) are $\left\{x_{1}^{l}, x_{1}^{l-1} x_{2}\right\}$. Therefore the principal terms of order $l$ in $x$ that remain in the normal form are of the type $\left(0, x_{1}^{l}\right)^{T}$ and $\left(0, x_{1}^{l-1} x_{2}\right)^{T}$ and in the lowest order

$$
\left(\begin{array}{c}
\dot{x}_{1} \\
\dot{x}_{2}
\end{array}\right)=\left(\begin{array}{c}
x_{2} \\
0
\end{array}\right)+\left(\begin{array}{c}
0 \\
v^{[2]} x_{1}^{2}+v^{[1]} x_{1} x_{2}
\end{array}\right)+\cdots
$$

The solution of (75),

$$
w^{\{l, m\}}(x, z)=z^{\{m\}}\left(\begin{array}{c}
\sum_{l_{1}=2}^{l} \underbrace{w_{1}^{\left[l_{1}\right]} x_{1}^{l_{1}} x_{2}^{l-l_{1}}}_{\mathrm{Im} \mathfrak{P}^{2}}+\underbrace{w_{1}^{[1]} x_{1} x_{2}^{l-1}+w_{1}^{[0]} x_{2}^{l}}_{\mathrm{Ker} \mathfrak{P}^{2}} \\
\sum_{l_{1}=0}^{l} w_{2}^{\left[l_{1}\right]} x_{1}^{l_{1}} x_{2}^{l-l_{1}} \\
\vdots
\end{array}\right),
$$

has two undetermined parameters, $w_{1}^{[1]}$ and $w_{1}^{[0]}$. [The notation is as in (76): $w_{j}^{\left[l_{1}\right]}$ is the coefficient of $x_{1}^{l_{1}} x_{2}^{l-l_{1}}$ in the $j$ th row of $w$.] The latter can, for instance, be fixed to zero but we should remember that even though these two terms do not contribute to the current main order of the transformation (75), say $r=l+m-1$, they affect higher orders $s>r$ (next rows of Lie triangle). In other words, $\tilde{u}_{s}>r$ depends on what values these parameters take. Below we show how crucial this dependence can be.

\section{Symplectic property}

Before we analyze the resonance terms in more detail we must consider how to make our transformation symplectic. In the faithful case $\mathrm{KerP}=\varnothing$ we should not worry because all we do is solve Eqs. (75) and eliminate the entire $u(x, z)$ : our solution $w(x, z)$ will replicate the Hamiltonian property of $\widetilde{u}(x, z)$. For $\operatorname{KerP} \neq \varnothing$ the situation requires special attention. In the Hamiltonian case variables $\left(x_{1}, x_{2}\right)$ [as well as $\left.\left(z_{j}, z_{j+1}\right), j=3,5, \ldots,(2 N-1)\right]$ are canonical conjugates, and at each order $r$ of the Lie transformation the components $w_{j}$ of the generator $w(x, z)$ must obey an additional relation

$$
\begin{aligned}
\frac{\partial^{2} W(x)}{\partial x_{1} \partial x_{2}} & =\frac{\partial w_{1}(x)}{\partial x_{1}}=w_{1}^{[1]} x_{2}^{l-1}+\cdots \\
& =-\frac{\partial w_{2}(x)}{\partial x_{2}}=-l w_{2}^{[0]} x_{2}^{l-1}+\cdots
\end{aligned}
$$

[cf. Eqs. (57)] such that in particular the coefficients are related by

$$
w_{1}^{[1]}=-l w_{2}^{[0]}
$$

Therefore, in the Hamiltonian case the parameter $w_{1}^{[1]}$ is determined by the symplectic condition (80c) together with the Lie equation (75). If we do not satisfy Eq. (80c) the trans- formed field of order $r$ will still be Hamiltonian, but higher orders will be irreversibly spoiled.

In contrast, there is no restriction due to (80) on parameter $w_{1}^{[0]}$ : the rank of the problem is $l-1$ and this parameter remains completely undetermined. This raises the question whether the normal form, our final result, is affected by $w_{1}^{[0]}$. To show that the normal form is indeed unique consider $\tilde{u}_{a}=\mathfrak{L}\left(w_{r}, u_{s}\right)$ with $w_{r}=\left(x_{2}^{l}, 0\right)^{T}$, where subscripts $a$, $r, s$ represent the orders and $a=r+s-1$. The $\left(\tilde{u}_{a}\right)_{2}$ component of this bracket is (at least) of order $l>1$ in $x_{2}$ and hence $\left(w_{r}\right)_{1}^{[0]}$ does not contribute to terms $x_{1}^{a}$ or $x_{1}^{a-1} x_{2}$ in $\left(\tilde{u}_{a}\right)_{2}$. Since all other contributions can always be eliminated at stage $a$ of the transformation, the resulting normal form is unique.

\section{Normal form in the Hamiltonian case}

Such a symplectic $w$ preserves the Hamiltonian form of the equations. To see this apply Eq. (74b) to a Hamiltonian field with components $u_{1}$ and $u_{2}$ related as in (80) and verify that terms $x_{1}^{l} x_{2}$ in $\mathfrak{P} w_{2}$ match those in $u_{2}$. Since $w_{2}$ is a solution of Eq. (75a) apply $\mathfrak{P}$ to both sides of this equation, neglect $\mathfrak{P}^{2} w_{1}$ because it does not contribute to the terms of interest and use relation between $u_{1}$ and $u_{2}$ :

$$
\mathfrak{P} w_{2}=\mathfrak{P}^{2} w_{1}-\mathfrak{P} u_{1} \rightarrow 0-x_{2} \frac{\partial u_{1}}{\partial x_{1}}=x_{2} \frac{\partial u_{2}}{\partial x_{2}}
$$

Therefore by solving Eq. (75a) for a Hamiltonian field $u(x)$ we not only eliminate $x_{1}^{l}$ (and all other nonlinear terms) from the $\dot{x}_{1}$ row, but also remove the related term $-l x_{1}^{l-1} x_{2}$ from the $\dot{x}_{2}$ row. As a consequence we have no mixed terms $x_{1}^{l} x_{2}$ in the normal form (78). In other words

$$
\dot{x}_{2}=\sum_{l>1} v_{2}^{[l]} x_{1}^{l}
$$




$$
H_{\mathrm{NF}}\left(\varepsilon_{\text {crit }}\right)=\frac{1}{2} x_{2}^{2}-\frac{1}{3} v_{2}^{[2]} x_{1}^{3}+\cdots
$$

\section{Resonances $\Lambda=(\lambda, m)-\lambda_{k}=0$}

The above outlines the procedure for the singular case, based on matrix (72b) with $\alpha=0$. Now let us recall that we are dealing with ODE's that depend smoothly on a single parameter $\varepsilon$. Typically $\alpha(\varepsilon)$ has a simple zero at $\varepsilon_{\text {crit }}$ and typically the eigenvalues $\lambda_{j}\left(\varepsilon_{\text {crit }}\right)$ are irrational numbers. In a generic situation, therefore, at $\varepsilon_{\text {crit }}$ the only set $\{m\}$ such that $(\lambda, m)=0$ is $\{m\} \equiv\{0\}, z^{\{m\}}=1$. It follows that the null space of $\mathfrak{P}$, and the resulting terms in the normal form of $\left(v_{1}, v_{2}\right)^{T}$, are entirely independent of the variables $z_{3}, \ldots, z_{N}\left[\right.$ cf. Eq. (78)]. Furthermore, $\operatorname{Ker}\left(\mathfrak{P}-\lambda_{k}\right)=\varnothing$, so Eqs. (75c) can be solved to eliminate everything from the normal form: $v_{3}=v_{4}=\cdots=v_{N}=0$ and $\dot{z}=\lambda z$.

Essentially the same argument applies in a neighborhood of $\varepsilon_{\text {crit }}$. There are additional resonances $(\lambda, m)-\lambda_{k}=0$, but in a sufficiently small neighborhood of $\varepsilon_{\text {crit }}$ they are only high-order resonances. Therefore the normal form $v$ has the same low-order terms with coefficients depending on $\varepsilon$.

On the other hand, a Hamiltonian system always has "uninteresting" resonances (Sec. VII A 4), which mix the two subsystems $\left(x_{1}, x_{2}\right)$ and $\left(z_{3}, \ldots, z_{2 N}\right)$. Since $\lambda_{3}=-\lambda_{4}$, we will find such resonances $(\lambda, m)=0$ whenever $m_{3}=m_{4}$ and our nonremovable terms in $v_{2}$ must also include $x_{1}^{l}\left(z_{3} z_{4}\right)^{m_{3}}$. Further thought tells us that the normal form of $v$ contains

$$
v(x, z)=\mu\left(\begin{array}{c}
0 \\
-x_{1}^{l}\left(z_{3} z_{4}\right)^{a} \\
x_{1}^{l+1}\left(z_{3} z_{4}\right)^{a-1} z_{3} \\
-x_{1}^{l+1}\left(z_{3} z_{4}\right)^{a-1} z_{4} \\
\ldots
\end{array}\right),
$$

with $z_{3}=\overline{z_{4}}$ a canonical pair. These terms are more easily expressed in terms of the Hamilton function [for $\alpha=0$, cf. Eq. (81b)]

$$
H_{\mathrm{NF}}\left(x, I ; \varepsilon_{\text {crit }}\right)=\frac{1}{2} x_{2}^{2}-\frac{1}{3} v_{2}^{[2]}(I) x_{1}^{3}+\cdots,
$$

where $v_{2}^{[2]}(I)=v_{2}^{[2]}\left(I_{3}, I_{4}, \ldots, I_{N}\right)$ is a power series in $I_{j}=\frac{1}{2} z_{2 j-1} z_{2 j}$. In other words the "uninteresting" resonances lead to a Birkhoff-type dependence of the terms of the resulting normal form on $N-1$ action variables $I_{j}$ of the nonsingular subsystem.

\section{Time dependence}

The main advantage of the method we present is that once time $\theta$ is replaced by auxiliary variables (48b) the latter are treated within the framework already developed for dynamical variables (albeit that they are regarded as parameters when the symplectic property is considered). The auxiliary system itself does not need to be transformed, and it follows that the terms left in the normal form of the main system include auxiliary variables as factors $\left(\zeta_{1} \zeta_{2}\right) \equiv 1$; i.e., the resulting normal form is time independent. Thus to study a periodic trajectory of a system with two degrees of freedom we transform the initial periodic system of two equations in $\left(x_{1}, x_{2}\right)$ into a system of four equations in $\left(x_{1}, x_{2}, \zeta_{1}, \zeta_{2}\right)$ and then reduce the latter to form $(81 \mathrm{~b})$.

\section{Real transformation}

One technical detail proves to be quite useful. If (some of) the eigenvalues $\lambda_{k}$ of matrix (72b) are imaginary (elliptic) we can avoid complex arithmetic by making the singular block imaginary:

$$
\begin{gathered}
x=\left(\begin{array}{ll}
1 & 0 \\
0 & i
\end{array}\right) y, \\
\dot{y}=\left(\begin{array}{cc}
1 & 0 \\
0 & -i
\end{array}\right) A\left(\begin{array}{cc}
1 & 0 \\
0 & i
\end{array}\right) y+\left(\begin{array}{cc}
1 & 0 \\
0 & -i
\end{array}\right) u(x(y)) \\
=i\left(\begin{array}{cc}
0 & 1 \\
-\alpha & 0
\end{array}\right) y+\left(\begin{array}{c}
u_{1}\left(y_{1}, i y_{2}\right) \\
-i u_{2}\left(y_{1}, i y_{2}\right)
\end{array}\right) .
\end{gathered}
$$

There is an important class of Hamilton functions of concrete physical systems that are (i) real and (ii) even in the momentum $p=x_{2}$ (often called time-reversal invariant). If $u(x)$ is a field generated by such a function then polynomials $u_{1}$ and $u_{2}$ are, respectively, odd and even functions of $x_{2}$ so that the transform of $u(x)$ on the rhs of Eq. (84b) is purely imaginary. In such a case $i$ becomes an overall factor and we use real polynomials in $(y, z)$ for the Lie transformation of field $u$.

\section{Normal form in the $C_{1}$ case}

In this case the coordinate system $\left(x_{1}, x_{2}\right)$ can be chosen such that the linear part $A x$ is always singular, i.e., $\alpha \equiv 0$ (see Sec. II B 4). On the other hand, the field at the origin is nonsingular for all parameter values $\varepsilon \neq \varepsilon_{\text {crit }}$; i.e., the field has a constant component $u_{\text {const }} \propto \varepsilon-\varepsilon_{\text {crit }}$, which vanishes at the critical point. The normal form of the Hamilton function is

$$
H_{\mathrm{NF}}(x, I ; \varepsilon)=a\left(\varepsilon-\varepsilon_{\text {crit }}\right) x_{1}+\frac{1}{2} x_{2}^{2}+\frac{1}{3} v_{\varepsilon}(I) x_{1}^{3}+\cdots
$$

(cf. Table I). Note that the dependence of nonlinear terms $v_{\varepsilon}(I)$ on $\varepsilon$ has no qualitative significance; the critical term is $a\left(\varepsilon-\varepsilon_{\text {crit }}\right) x_{1}$.

\section{9. $C_{2}$ case: solution for $\alpha \sim 0$}

The $C_{2}$ case differs from the above in that (i) the origin is always a fixed point; (ii) matrix $A$ of the linear part becomes singular only at $\varepsilon_{\text {crit }}$, which is a simple zero of $\alpha(\varepsilon)$; and (iii) the Hamilton function is an even function of both $x_{2}$ and $x_{1}$. The main technical problem arises here due to nonzero $\alpha$. Instead of Eqs. (74b) we have

$$
\begin{gathered}
\left(\mathfrak{P}+\alpha \mathfrak{P}_{2}\right) w_{1}-w_{2}=u_{1}, \\
\left(\mathfrak{P}+\alpha \mathfrak{P}_{2}\right) w_{2}-\alpha w_{1}=u_{2}, \\
\left(\mathfrak{P}+\alpha \mathfrak{P}_{2}\right) w_{k}-\lambda_{k} w_{k}=u_{k},
\end{gathered}
$$

with $\mathfrak{P}$ defined in Eq. (74c) and 

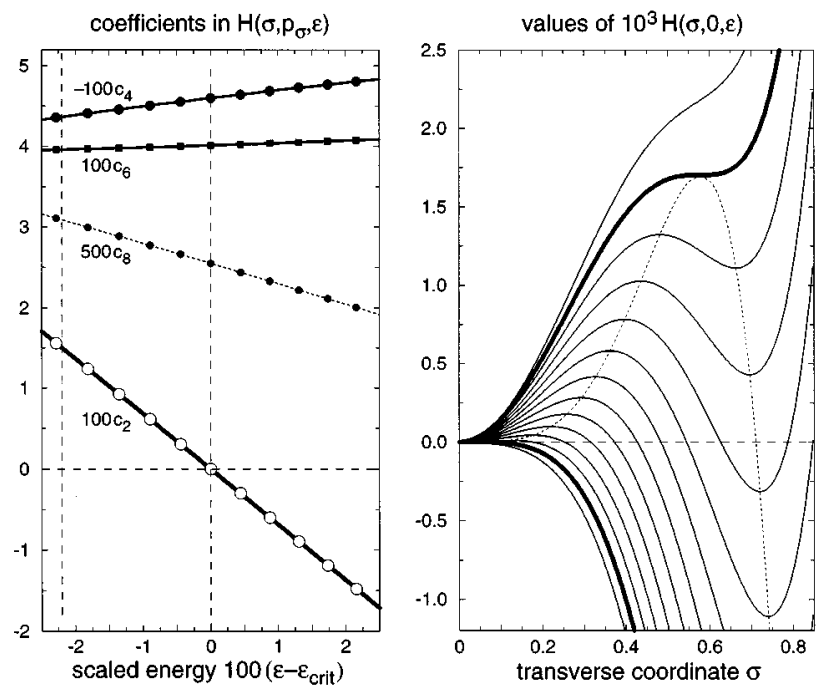

FIG. 16. Normal form (88a) near the period-1 $C_{2}$ symmetric (pitchfork) bifurcation of the perpendicular orbit. Left: dependence of coefficients on scaled energy $\varepsilon$, with $\varepsilon_{\text {crit }}=-0.12726$. Comparing to Eq. (88a), $c_{2}$ is $\alpha\left(\varepsilon-\varepsilon_{\text {crit }}\right.$ ), and $c_{4}, c_{6}, c_{8}$ are, respectively, $v_{1}, v_{2}, v_{3}$. Right: sections at $p_{\sigma}=0$ and $\varepsilon-\varepsilon_{\text {crit }}=-0.024$ (top), -0.022014 (saddle node), $-0.020,-0.018, \ldots, 0,0.002$.

$$
\mathfrak{P}_{2}=x_{1} \frac{\partial}{\partial x_{2}} \text {. }
$$

We solve Eqs. (86) in the same way as Eqs. (75): we entirely eliminate $u_{1}$ and determine $w_{2}$ from Eq. (86a); substitute $w_{2}$ into Eq. (86b) to obtain an equation for $w_{1}$; construct components $w_{1}$ and $w_{k}$ from homogeneous polynomials (79) in $\operatorname{Im}^{2}$ and $\operatorname{Im} \mathfrak{P}$ as we did for $\alpha=0$. In other words in the case of resonance we reduce the dimension of the system as if $\alpha=0$ with the only difference being that matrices replacing (77) and (76) have many additional small nonzero elements. Naturally, this is the only stable procedure for $\alpha \sim 0$.

As a result

$$
H_{\mathrm{NF}}(x, I ; \varepsilon)=\alpha(\varepsilon) x_{1}^{2}+\frac{1}{2} x_{2}^{2}+\frac{1}{4} v_{\varepsilon}(I) x_{1}^{4}+\cdots .
$$

\section{B. Symmetric period-1 bifurcation of the perpendicular orbit}

Due to the $\mathrm{R}_{\sigma}$ symmetry of the perpendicular orbit the period-1 bifurcation is not of the generic "saddle-node" or $C_{1}$ type in Table I but of the "pitchfork" or $C_{2}$ type. Symmetry saves the life of this orbit. The $C_{2}$-symmetric normal form is

$$
H_{\mathrm{NF}}=\frac{1}{2} \alpha\left(\varepsilon-\varepsilon_{\text {crit }}\right) \widetilde{\sigma}^{2}+\frac{1}{2} \widetilde{p}_{\sigma}^{2}+\sum_{j=1} v_{j}(\varepsilon) \widetilde{\sigma}^{2 j+2}
$$

with $\varepsilon_{\text {crit }}=-0.12726$. To achieve such a canonical representation we scaled the initial coordinates so that

$$
\left(\widetilde{\sigma}, \tilde{p}_{\sigma}\right)=\left(\sigma \mu(\varepsilon)^{1 / 2}, p_{\sigma} \mu(\varepsilon)^{-1 / 2}\right),
$$

with effective mass $\mu(\varepsilon)$ well approximated as $\mu(\varepsilon)=0.42719+0.12172\left(\varepsilon-\varepsilon_{\text {crit }}\right)$. As shown in Fig. 16 (left) parameters in (88a) are also essentially linear functions of $\left(\varepsilon-\varepsilon_{\text {crit }}\right)$, and, of course, $v_{0}\left(\varepsilon_{\text {crit }}\right)=\frac{1}{2} \alpha(0)=0$.
TABLE V. Parameters of the normal form (88a) near the period-1 symmetric bifurcation of the perpendicular orbit as series in scaled energy $\varepsilon$.

\begin{tabular}{ccccc}
\hline \hline & $f^{\mathrm{a}}$ & $f^{(0)}$ & $f^{(1)}$ & $f^{(2)}$ \\
\hline$q^{2}$ & $\alpha / 2$ & 0 & -6.848360 & -1.410814 \\
$q^{4}$ & $v_{1}$ & -0.460116 & -1.011357 & 5.491070 \\
$q^{6}$ & $v_{2}$ & 0.401166 & 0.273090 & 2.851380 \\
$q^{8}$ & $v_{3}$ & 0.050968 & 0.498350 & -0.506682 \\
\hline \hline
\end{tabular}

${ }^{\mathrm{a}} f\left(\varepsilon=\left[f^{(0)}+f^{(1)}\left(\varepsilon-\varepsilon_{\text {crit }}\right)+1 / 2 f^{(2)}\left(\varepsilon-\varepsilon_{\text {crit }}\right)^{2}\right] \times 10^{-1}\right.$.

\section{Organized bifurcations}

The numeric values of the parameters we obtained for (88a) are listed in Table $\mathrm{V}$. The major nonlinear contribution $v_{1} \sigma^{4}$ has negative sign, while the next term $v_{2} \sigma^{6}$ is positive. This results in the organization phenomenon described in Sec. II A 1, Eq. (2c). And indeed, as shown on the $p_{\sigma}=0$ sections in Fig. 16 (cf. Fig. 2), at first a saddle-node bifurcation occurs at $\varepsilon \approx-0.149\left(\varepsilon-\varepsilon_{\text {crit }} \approx-0.022\right)$ and $\sigma>0$. Due to the $\mathrm{R}_{\sigma}$ symmetry a twin saddle-node bifurcation occurs at $\sigma<0$. This produces two stable points (minima) and two unstable points (saddles). As $\varepsilon$ increases the unstable points move towards the origin, collide there, and disappear at $\varepsilon_{\text {crit }}$. Figure 17 illustrates this process.

The scale of the described phenomenon is large, in terms of both $\sigma$ and the range of $\varepsilon$. Moreover, from Fig. 9 we find that in the range $\varepsilon \approx-0.149 \cdots \varepsilon_{\text {crit }}$ the perpendicular orbit has many high-order resonances with $k>10$. Furthermore, at such high $\varepsilon$ the motion is very irregular: most of the phase space is chaotic. All this would be a good excuse for the normal form (88a) to fail. It needs no mercy, however. While the $v_{1}$ and $v_{2}$ coefficients in Table $\mathrm{V}$ are of the same magnitude, the value of $v_{3}$ drops significantly, thus indicating that the formal series can be extended to $q^{8}$ and even to $q^{10}$. (And indeed the period- 8 resonance is sufficiently distant in $\varepsilon$.) Using the three terms $q^{4}, q^{6}$, and $q^{8}$ we obtain the value of -0.1490 , while the numerical estimate (from the sequence of the Poincare surfaces) made for us by Shaw [11] gives -0.1482 . Intrigued by such success of the normal form approach we compared the simple prediction in Fig. 17 with the actual surface of section in the whole $\left(\sigma, p_{\sigma}\right)$ domain of interest. This section, also provided by Shaw [10], demonstrates both the power and the limitation of the normal form. The global picture is indeed very similar to that predicted by the simple contour plot in Fig. 17. In terms of the positions of the stationary points and the dimensions of the stable islands the normal form is quantitatively correct. (To compare, place the surface of section between the bottom and the middle contour plots according to $-0.015<\varepsilon-\varepsilon_{\text {crit }}=-0.0127<0$.) However, the normal form cannot reproduce all the fascinating destruction caused by other resonances. As we see in Fig. 18, the stable twins undergo a period-6 bifurcation of their own; a barely seen chain below and above the central stable island may also indicate a high-order resonance of the central orbit. At close distance we witness the structure of the emerging chaos.

\section{Periodic orbits and their symmetry}

This period-1 bifurcation of the half map corresponds to a period-1 bifurcation of the full map. The twin stationary 


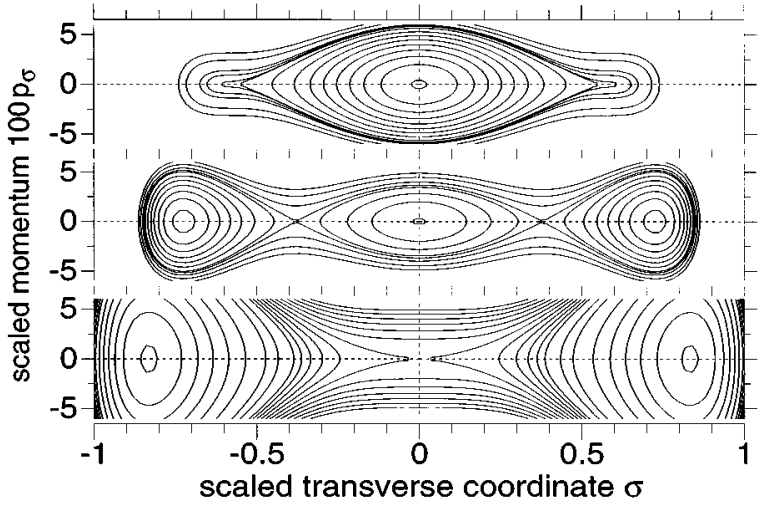

FIG. 17. Contour plot of the normal form $H_{\varepsilon}\left(\sigma, p_{\sigma}\right)$ near the $C_{2}$ symmetric period-1 bifurcation of the perpendicular orbit: $\varepsilon-\varepsilon_{\text {crit }}=-0.022$ (top), -0.015 (middle), 0.002 (bottom).

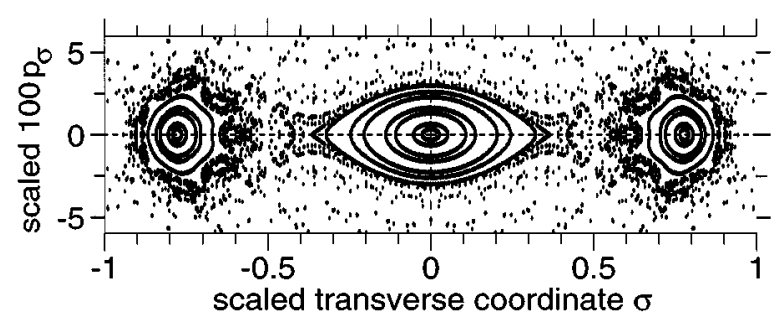

FIG. 18. Poincaré surface of section near the $C_{2}$ symmetric period-1 bifurcation of the perpendicular orbit: $\varepsilon=-0.14$, $\varepsilon-\varepsilon_{\text {crit }}=-0.01271$. The data were provided by J. Shaw.

points of the normal form (Fig. 17) are not connected by the map: each point corresponds to a distinct periodic orbit in $\left(u^{\prime}, v^{\prime}\right)$. The four orbits are shown in Fig. 19. The twins are related to each other by $\mathrm{R}_{\sigma}$. Hence, similar to all odd- $k$ bifurcations they break the $\mathrm{R}_{\sigma}$ symmetry and preserve $\mathrm{R}_{\lambda}$. Each orbit passes the surface of section in both directions $\left(p_{\lambda} \gtrless 0\right)$, and is a line in $\left(u^{\prime}, v^{\prime}\right)$ (is degenerate). The unstable twins shown in Fig. 19 move to the perpendicular trajectory as $\varepsilon \rightarrow \varepsilon_{\text {crit }}$ and disappear. None of these trajectories passes through the origin.

\section{CONCLUSIONS}

\section{A. Results}

Normal form theory was sketched by Poincare and then by Birkhoff nearly 70 years ago; since then it has become a standard tool for qualitative analysis. Normalization about an equilibrium point has been used as a quantitative tool for many years. Normalization about a periodic orbit is more complex. This paper reports the first full numerical implementation of this procedure as a quantitative scheme for studying developments in the phase space near a PO.

Normalization about a PO proceeds in three steps. (i) Isoenergetic reduction: we choose coordinates along and across the original $\mathrm{PO}$, and reduce $2 \mathrm{~N}$ autonomous equations to $2 N-2$ nonautonomous equations. (ii) Floquet transformation: we make a linear periodic change of variables to eliminate the time dependence in the linear equations. (iii) Lie transformation: we construct Lie generators of a sequence of

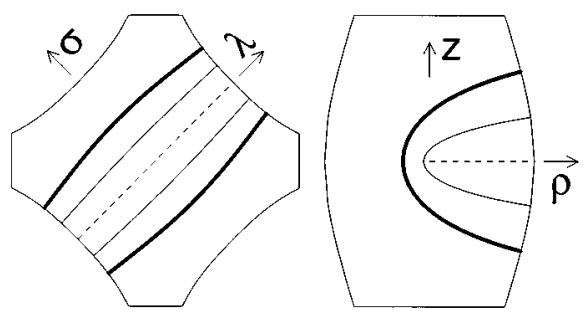

FIG. 19. Periodic trajectories created near the perpendicular orbit before it undergoes the period-1 ("last") bifurcation and becomes unstable in the $(u, v)$ (left) and $(\rho, z)$ coordinates for scaled energy $\varepsilon=-0.14$. Stable trajectory is shown by a bold line.

canonical transformations to eliminate all possible nonlinear terms from the equations, leaving only those that are essential. This transformation is terminated at a desired order and the resulting truncated normal form is a Hamilton function, which defines integrable dynamics. This function has the property that its contour plots correspond to Poincare surfaces of section of the original system: in particular, PO's of the original system correspond to stationary points of the normal form, and bifurcations of PO's are understood as bifurcations of these stationary points.

The normal form method is a practical and effective method for studying motion in the vicinity of a PO. As the present paper indicates, some effort is required to construct and implement a normalizing algorithm; however, once that is done, the normal form gives back information about PO's in an efficient, compact, and eloquent manner. For example, we have found that pictures such as Fig. 1 are generated at least ten times faster by normalization than by numerical construction of surfaces of section.

More important, of course, is that the simple structure of normal forms provides insight and understanding that cannot be obtained simply from numerical integration of trajectories. At the linear level the method tells us at which values of parameter $\varepsilon$ bifurcations will occur. Extending the normal form to higher degrees, we can now understand the organized sequences of bifurcations that had previously been seen in calculations. We can also see that the organized sequence of bifurcations shown in Fig. 1 is not some strange anomaly that is unique to the perpendicular PO of diamagnetic Kepler problem. It must occur commonly-the normal form predicts such behavior whenever the major nonlinear terms in the normal form have opposite signs, and provided that higher-order terms are small in the region of interest. This occurs for all 1:k bifurcations of the perpendicular PO with the exception of the 1:3 bifurcation (Tables IV and V). It will occur in many other systems.

Finally, the theory and the examples lead in new directions, some of which are suggested below.

\section{B. Connections with other work and perspectives for future study}

\section{Normal forms, integrability, and chaos}

The relation between normal-form theory and chaotic motion is well represented in Figs. 17 and 18. If the sequence of canonical transformations that generates the normal form were to converge then the system would have only integrable motion, no chaos. It is well known, however, that in general 
that sequence diverges $[1,14,29]$. Truncation of this normal series is the main simplification, which replaces the true Hamiltonian by an integrable one. Despite the fact that this integrable system cannot be equivalent to the initial system (their phase portraits, or families of phase curves, are topologically different), the theory proves to be quite a powerful tool. Normal form theory produces a finite precision local approximation that is valid in an average (short time) sense and that discards chaotic behavior ([29], Appendix 7.E). The way this theory functions is to some extent similar to the way our eye analyzes numerical Poincaré surfaces of section: it suppresses (eliminates) chaotic "noise," brightens regular features (islands of stability), interpolates these features, i.e., interpolates the Poincare map (in particular removes separatrix splitting), and finally reshapes the picture into an ideal $C_{k}$ symmetric form, such as in Fig. 1 and Table I.

However, limited normal form theory is for any global (phase space and time) analysis of nonintegrable systems, it perfectly suits the purpose of studying the qualitative changes in the local short-time behavior, such as bifurcations of periodic orbits or equilibria. For such a study we consider the normal form as a formal series and take the few first terms. This model is equivalent to the initial system only in terms of formal series. For those who like to start from the surfaces of section the equivalence relation (periodic orbits) $\sim$ (fixed points of the Poincare map) $\approx$ (fixed points of the normal form of this map) (stationary points of effective Hamiltonian) would give the main idea of this paper. We may conclude that the normal-form method is a tool to study short-time correlations in the phase space of a system with soft chaos.

\section{Dynamical vs a priori symmetry}

Our problem (DKP) is typical of systems studied by physicists in that it contains a priori symmetries in addition to the approximate symmetries caused by the resonances and idealized in the normal form. In this paper we treated these $a$ priori symmetries in an elementary and $a d$ hoc manner. It is clear that a more general and systematic treatment can be done and would be helpful. An important step in that direction was made by de Aguiar et al. [12]: considering symmetric maps they showed that a bifurcation of a $\mathrm{PO}$ in a $C_{s}$-symmetric system produces a $2 k$-island chain. More remains to be done. Normal-form theory has a great potential for application in the analysis of problems with symmetry. A systematic analysis would show how this symmetry gets built into the symmetry of the normal form; i.e., it would start from the total symmetry group $\mathfrak{G}$ of the total initial Hamilton function, select those symmetry operations that form a symmetry group $\mathfrak{g} \subset \mathfrak{G}$ of the periodic solution under study, and define the action of $\mathfrak{g}$ on this solution and on the surrounding phase space. We plan to present such an approach in a future paper.

\section{Higher dimensions}

In this paper we used as an example a system with two degrees of freedom. Nevertheless, we formulated the theory in general terms for systems with $N$ degrees of freedom. We believe that the real power of normal form methods will be manifested in systems with $N=3$ or 4 . The Poincare surfaces of section for such systems are of dimension 4 or 6 and are hard to generate numerically and difficult to envision and to comprehend.

In contrast, implementation of the normal-form theory is straightforward. Thus for $N=3$, normalization about a PO produces an effective Hamiltonian with $N=2$, and this can be studied by standard methods. Moreover, near each bifurcation the normal-form method selects an appropriate observation plane in the four-dimensional phase space of this Hamiltonian. Atoms in crossed fields are obvious candidates for such studies.

\section{Connections with quantum descriptions}

All of this theory arose because of experimental measurements on a quantum system [8]. What connections exist between normal-form theory and quantum Hamiltonians and wave functions?

For systems with (approximate) integrals, semiclassical quantization can be based on the global normal form of the Hamilton function near equilibrium (see Refs. [3], Chap. 14.3, [44-46], and many others).

Nonintegrable systems can be quantized according to Gutzwiller's theory [3], which shows that quantum mechanics develops around periodic orbits of the classical problem. Due to the uncertainty principle (due to the finite resolution, the "speck" structure of quantum phase space) classical dynamics near these orbits is time-space averaged in essentially the same way as in the normal form: over finite (short) time and locally. Thus the absence of classical chaos in the normal form is somewhat analogous to that in the quantum problem. It follows that local normal forms near periodic orbits that we work with in this paper are directly related to the semiclassical theory of chaotic systems.

For example, experimental measurements on atoms in fields show quantum manifestations of bifurcations of periodic orbits. Near a bifurcation, semiclassical formulas for recurrence strengths and the density of states diverge, and have to be replaced by uniform approximations [47]. The information needed to construct these approximations is, in fact, contained in the normal form.

\section{ACKNOWLEDGMENTS}

This work was supported by the Office of Naval Research and the National Science Foundation through grants to the College of William \& Mary. We thank Joint Institute for Laboratory Astrophysics for hospitality. We thank John Shaw for discussions, for providing $\left(u^{\prime}, v^{\prime}\right)$ plots of trajectories in Figs. 19, 15(a), and 13(a), and for making his numerical calculations for the trace (solid lines in Fig. 9) and for the Poincare surface of section in Fig. 18 available for comparison. We also thank K. Meyer for discussion and inspiration, and B. Zhilinskií for many helpful comments.

\section{APPENDIX A: HAMILTON FUNCTIONS, VECTOR FIELDS, AND MAPS}

Qualitative theory of dynamical (Hamiltonian) systems can be equivalently developed in terms of Hamilton functions, Hamiltonian vector fields, and symplectic maps. Normal form theory gives a technique to reduce any of these 
three representations of the system to some canonical simple form with only essential (nonreducible) terms remaining.

The language of vector fields unifies the Hamiltonian case with the general theory of differential equations. It is particularly convenient for the theory of time-dependent or driven systems. Since the equations of motion near a periodic orbit are of this latter type, we primarily use this language in this paper. On the other hand, the use of Hamilton functions takes full advantage of the symplectic nature of the problem, is compact, eloquent, and hence the most convenient for presenting the results, such as Eq. (1) and Fig. 1. Maps have been much appreciated since Poincaré. They are more illustrative and more familiar in applications, but are less convenient for our purposes.

\section{Hamilton functions near equilibrium}

Qualitative theory for one degree of freedom directly follows from Sec. II A 2. We use $x=(q, p)$, or $(\varphi, I)$, with $I=\frac{1}{2}\left(q^{2}+p^{2}\right)=\frac{1}{2} \rho^{2}$, as symplectic (canonical) coordinates on the plane and consider $f_{\varepsilon}(q, p)$ or $f_{\varepsilon}(-\varphi, I)$ in Table I as Morse (generic) Hamilton functions. We choose the origin as described in Sec. II B 4 and obtain linearized equations of motion $\dot{x}=A_{\varepsilon} x$ with Jacobian matrix

$$
A_{\varepsilon}=\left(\begin{array}{cc}
0 & 1 \\
-1 & 0
\end{array}\right)\left(\frac{\partial^{2} f_{\varepsilon}}{\partial(q, p)}\right),
$$

whose determinant and hence singularity properties are the same as those of (3b). For $N$ degrees of freedom $A_{\varepsilon}$ is a Hamiltonian matrix: $A_{\varepsilon} \in \operatorname{sp}(N, r)$ ([16], Chap. II A), the theory extends naturally [28].

\section{Hamiltonian vector fields near a singular point}

In one degree of freedom we again implement Morse functions in Table I to generate qualitatively different possible normal forms of generic (Hamiltonian) vector fields on the plane near their singular point

$$
v_{\varepsilon}(q, p)=\left(\begin{array}{cc}
0 & 1 \\
-1 & 0
\end{array}\right)\left(\frac{\partial f_{\varepsilon}}{\partial q}, \frac{\partial f_{\varepsilon}}{\partial p}\right)^{T} .
$$

Fields $v_{\varepsilon}$ are equivariant: they commute with operations of the corresponding symmetry group $C_{k}$ [32].

The matrix of the linearized field (A1) defines the direction of the field (direction of motion, or time evolution) near the origin. The phase portraits can be relatively simply understood from the contour plots (constant energy sets) in Table I. The field is tangent to these contours and its direction can be indicated by the "direction of the contour" as shown in Fig. 1(d). The stationary points (3a) of $f_{\varepsilon}$ become the singular points of $v_{\varepsilon}$. To determine relative directions we choose an arbitrary direction for one nonseparatrix contour and assign the same (the opposite) direction to all other nonseparatrix contours whose points can be connected to those of the initial one by a continuous line that crosses an even (odd) number of separatrices (and avoids fixed points). Reversing time $t \mapsto-t$ simultaneously reverses all directions.

An important point to note is that the symmetry of the fields (of the phase portraits and of the directed contours) is precisely $C_{k}$ [32], the one indicated in Table I. This is be- cause reflection, such as $\sigma:(q, p) \mapsto(q,-p)$, is not a symplectic (canonical) transformation and hence does not commute with Hamiltonian fields. (Similar to reversing time reflections change the sign of $d q \wedge d p$.)

\section{Symplectic maps of the plane near a fixed point}

Symplectic maps naturally arise in dynamical theory as Poincaré maps (see [17], Sec. 6.32D and [16], Chap. V E) and as such are often used in the study of periodic orbits. Thus Meyer obtained his classification [9] (see Sec. II B) by studying generic bifurcations of symplectic maps of the plane and using normal forms of such maps near a fixed point.

Of course, Meyer's normal forms of the maps (of area preserving diffeomorphisms of the plane) are finite difference analogs of (A2) and can be derived from Table I. For instance, the flow

$$
\left(\begin{array}{c}
\dot{q} \\
\dot{p}
\end{array}\right)=\left(\begin{array}{c}
p+\cdots \\
-\varepsilon-q^{2}+\ldots
\end{array}\right),
$$

generated by the the $C_{1}$-type Morse Hamiltonian, corresponds to Meyer's extremal case ([16], Chap. VIII A2)

$$
\left(\begin{array}{l}
q \\
p
\end{array}\right) \mapsto\left(\begin{array}{l}
q \\
p
\end{array}\right)-\varepsilon+\left(\begin{array}{ll}
0 & 1 \\
0 & 0
\end{array}\right)\left(\begin{array}{l}
q \\
p
\end{array}\right)-\left(\begin{array}{c}
\ldots \\
q^{2}+\ldots
\end{array}\right) .
$$

The map near the transitional fixed point ([16], Chap. VIII A 3) corresponds to the $C_{2}$ Morse function. For $k$-bifurcation points ([16], Chap. VIII A 4) $(k>2)$ we use angle-action coordinates (50a). This produces a timeindependent map in the frame $(\widetilde{\varphi}, \widetilde{I})$ rotating with rational frequency $n / k$. Explicit time dependence can be restored by (canonical) backsubstitution (cf. Sec. II B 5)

$$
(\widetilde{\varphi}, \widetilde{I})=(\varphi-n t / k, I), \quad F_{2}=I(\widetilde{\varphi}+n t / k) .
$$

The map is then produced by setting $t=2 \pi$ (the period).

We also note that the linear part and the nonsingularity condition of the map $z \mapsto z+A_{\varepsilon} z+\cdots$ are given by Eqs. (A1) and (3b). The contour plots in Table I give an image of the corresponding map ([16], Chap. VIII A 1). Like vector fields, maps are directed, and the only symmetry operations that can commute with them are of type $C_{k}$.

Truncated normal forms of symplectic maps $P$ and of Hamilton functions $H$ are indeed equivalent, and for studying local and short time phenomena such equivalence suffices. However, regarding $P$ and $H$ themselves, we can only say that at the level of formal series $P$ can be interpolated by the flow of $H$ ([17], Sec. 2.26.H, p. 200 and Sec. 6.35, p. 321).

\section{APPENDIX B: CURVILINEAR COORDINATES NEAR A TRAJECTORY IN TWO DEGREES OF FREEDOM}

Consider a smooth configuration-space curve $R(\lambda)$ parametrized by its natural parameter (length) $\lambda$ ([7], Appendix D1). In a simple case of dimension two, i.e., $R(\lambda)=\left[R_{x}(\lambda), R_{y}(\lambda)\right]$, the space of normal variations is defined by the normal unit vector $n:[v \times n]=1$, or $\left(n_{x}, n_{y}\right)=\left(-v_{y}, v_{x}\right)$, where $v=d R / d \lambda$ is the speed vector 
and $|v(\lambda)| \equiv 1$ since $\lambda$ is a natural parameter. The derivative of $n$ is given by the Frenet formula (see [48], Pt. I or [49], Chap. 15.4)

$$
\frac{d n}{d \lambda}=-\kappa(\lambda) v= \pm\left|\frac{d v}{d \lambda}\right| v
$$

where $\kappa(\lambda)$ is (signed) curvature. Radius vectors $r$ of points sufficiently close to the curve can always be uniquely represented as

$$
\begin{gathered}
r(\lambda, \sigma)=R(\lambda)+\sigma n(\lambda), \\
\frac{\partial r}{\partial \lambda}=v(\lambda)[1-\kappa(\lambda) \sigma], \\
\frac{\partial r}{\partial \sigma}=n(\lambda)
\end{gathered}
$$

if $\sigma$, the distance to the curve, is small, or more precisely

$$
\kappa(\lambda) \sigma<1
$$

Equations (B2) introduce configuration space coordinates $(\lambda, \sigma)$ such that $\delta \sigma$ is normal to the configuration-space image of the trajectory. For Hamiltonian systems we use a Mathieu transformation with generating function ([7], Eq. (D6)]

$$
F_{3}\left(p_{x}, p_{y} ; \lambda, \sigma\right)=-p_{x} x(\lambda, \sigma)-p_{y} y(\lambda, \sigma)
$$

such that new conjugate momenta

$$
p_{\lambda, \sigma}=\left[\frac{\partial r}{\partial(\lambda, \sigma)}\right]^{T} p_{x, y, \ldots}
$$

are obtained from the Jacobian in (B2b) and (B2c).

\section{APPENDIX C: DIAMAGNETIC KEPLER PROBLEM}

\section{Larmor reduction, cylindrical coordinates}

The total three-dimensional Hamiltonian of the hydrogen atom in the magnetic field has continuous symmetry $C_{\infty}$; projection $L_{z}$ of the orbital angular momentum of the electron ( $z$ is parallel to the field) and longitude $\varphi$ are the corresponding integral of motion and ignorable angle. In cylindrical coordinates $(\rho, z, \varphi)$, and in the frame rotating with Larmor frequency $\omega_{L}=\frac{1}{2}(B / c)$ a.u. the reduced Hamiltonian is

$$
\begin{gathered}
H=\frac{1}{2}\left[\left(p_{\rho}^{2}+p_{z}^{2}-\left(\rho^{2}+z^{2}\right)^{-1 / 2}+\rho^{2}+\ell^{2} \rho^{-2}\right]=\varepsilon,\right. \\
\ell=\frac{1}{2} L_{z}(B / c)^{1 / 3}
\end{gathered}
$$

where $\varepsilon$ is the "scaled energy" (see [6,50] and [3], Chap. 18.4).

We formally extend the domain of definition of $(\mathrm{C} 1)$ to $-\infty<\rho<\infty$. This 1:2 image of the initial $\rho \geqslant 0$ problem has a reflection symmetry $\rho \rightarrow-\rho$ in addition to the symmetry $z \rightarrow-z[51]$.

\section{Regularization: semiparabolic coordinates}

The main difficulty of the Hamiltonian (C1) is the Coulomb singularity $\left(\rho^{2}+z^{2}\right)^{-1}$. It can be removed in the socalled semiparabolic coordinates $[5,6]$

$$
\begin{gathered}
(\rho, z)=\left(2 u v, u^{2}-v^{2}\right)=\left(u^{\prime 2}-v^{\prime 2}, 2 u^{\prime} v^{\prime}\right), \\
\left(u^{\prime}, v^{\prime}\right)=\left(\frac{u+v}{\sqrt{2}}, \frac{u-v}{\sqrt{2}}\right) .
\end{gathered}
$$

If we scale time (the Levi-Civita transformation) and consider the important case $L_{z}=0$, (C1) becomes

$$
\begin{aligned}
H_{\varepsilon}= & \frac{1}{2}\left(p_{u}^{2}+p_{v}^{2}\right)+4\left(u^{2}+v^{2}\right)\left[2(u v)^{2}-\varepsilon+\frac{\widetilde{\ell}}{(u v)^{2}}\right]=2, \\
= & \frac{1}{2}\left(p_{u^{\prime}}^{2}+p_{v^{\prime}}^{2}\right)+4\left(u^{\prime 2}+v^{\prime 2}\right) \\
& \times\left[\frac{1}{2}\left(u^{\prime 2}-v^{\prime 2}\right)^{2}-\varepsilon+\frac{4 \widetilde{\ell}}{\left(u^{\prime 2}-v^{\prime 2}\right)^{2}}\right]=2,
\end{aligned}
$$

with $\varepsilon$ the only parameter. Because $L_{z}=0$ (C3) has no singularity [52].

To completely simplify the topology of the phase space we allow all values of $(u, v)$ in (C2) [51]. This gives a 4:1 image of the initial problem and causes many of the a priori symmetries of (C3). [The full symmetry group of (C3) is $D_{4}$, while the initial problem possesses only $z \mapsto-z$.] Nevertheless, the trajectories of the two problems are in a certain correspondence and hence we can study them for the smooth problem (C3) and then map onto those of the initial problem.

\section{Perpendicular orbit}

The perpendicular orbit is a particular solution for equations of motion defined by $(\mathrm{C} 1)$ such that $z \equiv 0$ and the corresponding Hamilton function is

$$
\mathcal{H}\left(p_{\rho}, \rho\right)=\frac{1}{2}\left(p_{\rho}^{2}+\rho^{2}\right)-\rho^{-1}=\varepsilon .
$$

In the coordinates (C2b) this becomes Eq. (16). In the extended $(\rho, z)$ frame we have two equivalent-by-symmetry solutions $\rho>0$ and $\rho<0$, which correspond to solutions $u^{\prime} \equiv 0$ and $v^{\prime} \equiv 0$. Obviously, we need to study only one of the solutions, and therefore we do not need to consider the $C_{4}:\left(u^{\prime}, v^{\prime}\right) \mapsto\left(v^{\prime},-u^{\prime}\right)$ symmetry of $(\mathrm{C} 3 \mathrm{~b})$.

\section{APPENDIX D: LOGARITHM OF A SYMPLECTIC $2 \times 2$ MATRIX}

In this appendix we construct the logarithm of a real symplectic $2 \times 2$ matrix $M \in \mathrm{Sp}(2, r)$. The logarithm is an element of the algebra $\operatorname{sp}(2, r)$, the algebra of generators of the group $\mathrm{Sp}(2, r)$.

Consider a real symplectic $2 \times 2$ matrix

$$
M=\left(\begin{array}{cc}
\alpha & \beta \\
\gamma & \delta
\end{array}\right) \in \operatorname{Sp}(2, r) \Leftrightarrow \operatorname{det} M=\alpha \delta-\beta \gamma=1, \quad \text { (D1a) }
$$

with eigenvalues 


$$
\lambda_{1,2}= \begin{cases}\exp ( \pm \kappa) & \text { for } \tau>-1 \\ \exp ( \pm \widetilde{\kappa}+i \pi) & \text { for } \tau<1\end{cases}
$$

with half trace

$$
\tau=\frac{1}{2} \operatorname{Tr} M=\frac{1}{2}(\alpha+\delta) .
$$

We connect matrix $M$ to the origin $I$ using the exponent

$$
M(t)=\exp (t \ln M), \quad M(0)=I, \quad M(1)=M .
$$

We will show that if $\tau>-1$, or in the "positive domain of $M$,' the $\log$ arithm $\ln M$ is a real Hamiltonian matrix, otherwise $\ln M$ cannot be real.

To define the logarithm of a symplectic $2 \times 2$ matrix (D1a) we construct a Hamiltonian matrix

$$
\begin{aligned}
\Omega & =\left(\begin{array}{cc}
(\alpha-\delta) / 2 & \beta \\
\gamma & (\delta-\alpha) / 2
\end{array}\right) \in \operatorname{sp}(2, r), \quad \operatorname{Tr} \Omega=0, \\
& =M-\tau I,
\end{aligned}
$$

or in terms of the generators of $\operatorname{Sp}(2)$,

$$
\Omega=\frac{\beta-\gamma}{2}\left(i \hat{\sigma}_{y}\right)+\frac{\beta+\gamma}{2} \hat{\sigma}_{x}+\frac{\alpha-\beta}{2} \hat{\sigma}_{z} \in \operatorname{sp}(2, r),
$$

where $\hat{\sigma}_{x}, \hat{\sigma}_{y}$ and $\hat{\sigma}_{z}$ are the Pauli matrices. Since

$$
\operatorname{det} \Omega=\sqrt{\tau^{2}-1},
$$

matrix $\Omega$ is factor $\tau^{2}-1$ idempotent (nilpotent for $|\tau|=1$ ),

$$
\Omega^{2}=\left(\tau^{2}-1\right) I
$$

We first consider the positive domain of $M$. Let $\kappa$ in (D1b) be

$$
\kappa=i \omega
$$

with $\omega$ real and positive for $-1<\tau<1$, zero for $\tau=1$, and positive imaginary for $\tau>1$. Using exponential definitions for hyperbolic and trigonometric functions, such as $\cosh \kappa=[\exp \kappa+\exp (-\kappa)] / 2$, we obtain

$$
\begin{gathered}
\tau=\cosh \kappa=\cos \omega, \\
\sqrt{\tau^{2}-1}=\sinh \kappa=i \sin \omega .
\end{gathered}
$$

It follows that $\kappa$ and $\sinh \kappa$ are real and positive if $\tau>1$, while $\omega$ and $\sin \omega$ are real and positive if $-1<\tau<1$. Then the logarithm of $M$ in (D1a) and (D2) can be expressed as

$$
\begin{aligned}
\ln M & =\frac{\kappa}{\sinh \kappa} \Omega=\frac{\omega}{\sin \omega} \Omega=\frac{\kappa}{\sqrt{\tau^{2}-1}} \Omega \text { for } \tau>-1 \\
& \equiv \Omega \text { for } \tau=1 .
\end{aligned}
$$

Proof. Using the property (D3d) of $\Omega$ makes exponentiation (D2) quite simple:

$$
\begin{aligned}
M(t) & =\exp \left(\frac{t \kappa}{\sqrt{\tau^{2}-1}} \Omega\right) \\
& =\sum_{m=0}^{\infty}\left[I \frac{(t \kappa)^{2 m}}{(2 m) !}+\frac{\Omega}{\sqrt{\tau^{2}-1}} \frac{(t \kappa)^{2 m+1}}{(2 m+1) !}\right] \\
& =I \cosh (t \kappa)+\frac{\sinh (t \kappa)}{\sinh \kappa} \Omega \text { for } \tau>-1 \\
& =I+t \Omega \text { for } \tau=1 .
\end{aligned}
$$

The above solution does not apply if $\tau<-1$. However, a different formula may be used in that domain, and indeed for all $\tau<1$. Write $\tilde{\kappa}$ in (D1b) as

$$
\widetilde{\kappa}=i \widetilde{\omega}
$$

with $\widetilde{\omega}$ real and positive for $\tau<-1$, zero for $\tau=-1$, and positive imaginary for $\tau<1$. Then

$$
\begin{gathered}
\tau=-\cosh \widetilde{\kappa}=-\cos \widetilde{\omega}, \\
\sqrt{\tau^{2}-1}=\sinh \widetilde{\kappa}=i \sin \widetilde{\omega},
\end{gathered}
$$

so that $\tilde{\kappa}$ and $\sinh \tilde{\kappa}$ are real and positive if $\tau<-1$, while $\widetilde{\omega}$ and $\sin \widetilde{\omega}$ are real and positive if $-1<\tau<1$. Then

$$
\begin{aligned}
\ln M & =\frac{-\widetilde{\kappa}}{\sinh \widetilde{\kappa}} \Omega+i \pi I=\frac{-\widetilde{\omega}}{\sin \widetilde{\omega}} \Omega+i \pi I \\
& =-\frac{\widetilde{\kappa}}{\sqrt{\tau^{2}-1}} \Omega+i \pi I \text { for } \tau<1 \\
& \equiv-\Omega+i \pi I \text { for } \tau=-1 .
\end{aligned}
$$

The proof is the same as before:

$$
\begin{aligned}
M(t) & =\exp \left(\frac{-t \widetilde{\kappa}}{\sqrt{\tau^{2}-1}} \Omega+i \pi t I\right) \\
& =\exp (i \pi t)\left[I \cosh (t \widetilde{\kappa})-\frac{\sinh (t \widetilde{\kappa})}{\sinh \widetilde{\kappa}} \Omega\right] \text { for } \tau<1 \\
& =\exp (i \pi t)(I-t \Omega) \text { for } \tau=-1
\end{aligned}
$$

The two logarithms (D4) and (D6) represent two different sheets of the logarithm function. They do not match in the domain of overlap $-1<\tau<1$.

We conclude that in accordance with general statements ([16], Chap. II) a family of real matrices $M(t)$ can be constructed for $\frac{1}{2} \operatorname{Tr} M>-1$ [38].

\section{APPENDIX E: ALTERNATIVE FORMULATIONS OF LIE TRANSFORMATION THEORY}

\section{Lie transformation of Hamilton functions and operators}

In Secs. VII and VI, we explain Lie transformation theory of vector fields-we worked directly with the differential equations (38). A similar Lie transformation theory can be 
used for Hamilton functions $[15,46]$ or (in quantum mechanics) for Hamiltonian operators [53]. (To highlight the correspondence we make the notation similar to that used in Secs. VII and VI.)

If our dynamical system is Hamiltonian, vector fields $u$, $w$, and $v$ in Eqs. (40) and (41) can be generated from Hamilton functions $U, W$, and $V$ [cf. Eq. (A2)]. The algorithm of Lie transformation $U \rightarrow V$ is the same as presented above, but with $\mathfrak{L}$ defined as the Poisson bracket

$$
\begin{aligned}
\mathfrak{L}(U, W)=\{U, W\} & =\nabla_{x} U\left(\begin{array}{cc}
0 & 1 \\
-1 & 0
\end{array}\right)\left(\nabla_{x} W\right)^{T} \\
& =\sum_{i=1}^{N}\left(\frac{\partial U}{\partial q_{i}} \frac{\partial W}{\partial p_{i}}-\frac{\partial U}{\partial p_{i}} \frac{\partial W}{\partial q_{i}}\right),
\end{aligned}
$$

so that the transformation generated by $W$ is symplectic.

The quantum analog of the theory of Lie transformations of classical Hamilton functions is the method of contact transformations (often named after Van Vleck in physics). In this latter theory we transform the quantum Hamiltonian

$$
\hat{U}=\hat{U}_{0}+\epsilon \hat{U}_{1}+\epsilon \hat{U}_{2}+\cdots,
$$

a self-adjoint operator on the Hilbert space, as

$$
\hat{U} \rightarrow \hat{V}=\hat{\mathfrak{W}}^{-1} \hat{U} \hat{\mathfrak{W}}
$$

using a transformation

$$
\hat{\mathfrak{W}}=\exp (i \hat{W})=\exp \left(\epsilon i \hat{W}_{1}+\epsilon^{2} i \hat{W}_{2}+\cdots\right),
$$

such that

$$
\hat{\mathfrak{W}}^{-1}=\hat{\mathfrak{W}}^{T} \Rightarrow \hat{W}^{T}=-\hat{W} .
$$

The Lie product, the quantum analog of (E1), is of course defined as $[A, B]=A B-B A$, and the transformed quantum Hamiltonian becomes

$$
\hat{V}=\hat{U}+[i \hat{W}, \hat{U}]+[i \hat{W},[i \hat{W}, \hat{U}]]+\cdots
$$

We substitute $\hat{U}$ and $\hat{W}$ as $\epsilon$ series (E2a) and (E2c) and collect terms of the same order-exactly as prescribed by the Lie triangle, to arrive at

$$
\hat{V}_{0}=\hat{U}_{0}, \quad \hat{V}_{1}=\hat{U}_{1}+i\left[\hat{W}_{1}, \hat{U}_{0}\right], \ldots .
$$

Thus if we want to modify (to eliminate) $\hat{U}_{1}$ we solve the Lie equation for $\hat{W}_{1}$.

As noted in Sec. IX B 1, projecting the quantum-classical correspondence on the problem of normal forms near a periodic trajectory is by itself a very interesting subject.

\section{Lie transformations periodic in time}

In Eqs. (48) we convert 2N-2 nonautonomous Hamiltonian equations into $2 \mathrm{~N}$ autonomous non-Hamiltonian equations. Two alternatives can be used.

a. Transformation of nonautonomous vector fields. We can operate with vector fields that explicitly depend on time. [Recall the slippery change from $\theta$ to $\left(q_{2}, p_{2}\right)$ in Eq. (10b) of
Sec. II B 5.] This, however, changes the Lie equation (43c) into

$$
\left(\mathfrak{L}_{A}+\frac{\partial}{\partial \theta}\right) w(y, \theta)=u(y, \theta),
$$

and incurs further changes to the finite transformation algorithm in Sec. VI A 2. The advantage of the periodicity in $\theta$ is taken when solving Eq. (E3): solution $w(y, \theta)$ is sought on invariant subspaces spanned by Fourier-Taylor terms $y^{m} \exp (i k \theta)$ ([17], Chap. 5, Sec. 25B).

b. Transformation of Hamilton function. If our equations are Hamiltonian we can make a $\theta$-dependent transformation of the Hamilton function $U(x, \theta, \epsilon)$ instead of the vector field $u(x, \theta, \epsilon)$. The Lie equation [cf. Eqs. (E3) and (E1)] becomes

$$
\{W, U\}+\frac{\partial}{\partial \theta} W(y, \theta)=U(y, \theta),
$$

and our new Hamilton function now includes an additional part, the reminder ([16], Chap. VII A 3), composed of derivatives $\partial W / \partial \theta$. Again, we make a general time-dependent transformation and take advantage of the periodicity in $\theta$ only when solving Eq. (E4) in terms of $y^{m} \exp (i k \theta)$.

\section{APPENDIX F: SYMMETRIES OF THE NORMAL FORM AND OF PERIODIC TRAJECTORIES IN 1: $k$ BIFURCATIONS}

A contour plot of the normal $H_{n: k}$ alone is not enough to understand completely the corresponding system of periodic trajectories. Detailed consideration for the perpendicular PO follows.

\section{Linear map properties}

We take into account that the coiling speed (linear frequency $\omega$ ) is $+n / k$ (the direction of the coil is clockwise) and the greatest common denominator $\operatorname{gcd}(n, k)=1$ so that orbits cross $\left(\sigma, p_{\sigma}\right) k$ times before the closure in $\left(\sigma, p_{\sigma}\right)$. From Eqs. (69) we easily conclude that if $k$ is even $H_{n: k}$ has $k$ stable and $k$ unstable stationary points and hence there is a stable-unstable pair of trajectories passing through these points. On the other hand, if $k$ is odd we have two such pairs: indeed, one stable (unstable) trajectory cannot pass all $2 k$ stable (unstable) points since it steps in $\varphi$ by $2 \pi n / k$, not by $\pi n / k$, and closes after $k$ crossings.

\section{Connecting pieces into wholes}

We should also remember that in Sec. V D we chose to use the half period, so that our trajectories return to $\left(\sigma, p_{\sigma}\right)$ at every half period $\theta=\pi l, l=0,1,2, \ldots$. Now we have to connect the halves. For this it is sufficient to remember that $p_{\lambda}>0$ when $\theta=2 \pi l$ and $p_{\lambda}<0$ when $\theta=\pi+2 \pi l$ [indeed $\left.p_{\lambda}=\sqrt{2 J_{\varepsilon}} \cos (\theta)\right]$. Thus if $k$ is even, such as $k=4$,

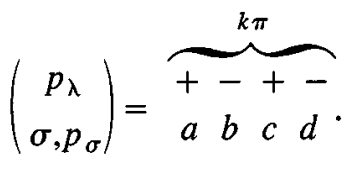

In other words, if $k$ is even we connect $k$ pieces into one whole, thus obtaining a period- $k / 2$ whole trajectory; a 
period- $k$ bifurcation of the half-period reduced problem turns out to be a period- $k / 2$ bifurcation of the actual trajectory $\left(\frac{1}{4}+\frac{1}{4}=\frac{1}{2}\right)$. On the other hand, if $k$ is odd, such as $k=3$,

$$
\left(\begin{array}{c}
p_{\lambda} \\
\sigma, p_{\sigma}
\end{array}\right)=\overbrace{+\begin{array}{llllll}
+ & - & + & - & + & - \\
a & b & c & a & b & c
\end{array},}^{2 k \pi},
$$

we have to connect $2 k$ pieces for the trajectory to retrace itself. Consequently, if $k$ is odd an $n: k$ bifurcation of the half-period problem corresponds to a $2 n: k$ bifurcation of the whole trajectory $\left(\frac{1}{3}+\frac{1}{3}=\frac{2}{3}\right)$.

\section{Behavior with respect to $R_{\sigma}$}

Resonances $n: k$ induce the $C_{k}$ symmetry in the $\left(\sigma, p_{\sigma}\right)$ plane. In the case of odd $k$ this symmetry is not compatible with $\mathrm{R}_{\sigma}=C_{2}$. Since the latter is inherent to the problem, the normal form $H_{n: k}$ centered at the perpendicular orbit has higher symmetry $C_{2 k}=C_{k} \otimes \mathrm{R}_{\sigma}$. The new periodic orbits (like the new stationary points of $H_{n: k}$ ) are, however, not $\mathrm{R}_{\sigma}$ invariant. They break this symmetry. On the contrary, in the even- $k$ case the new periodic trajectories remain $\mathrm{R}_{\sigma}$ invariant because $C_{k} \supset C_{2}$; i.e., the induced symmetry contains $\mathrm{R}_{\sigma}$.

The described symmetry properties can be easily observed if we represent trajectories by the groups of $k$ equivalent stationary points of $H_{n: k}$ [43]. (If such a set represents two different trajectories passing through the same points in the opposite directions, we do not need to distinguish them here because they transform in the same way with respect to $\mathrm{R}_{\sigma}$.) An odd- $k$ set of points, such as $\{2 \pi l / k\}$, $l=0,1, \ldots, \quad$ is not invariant under $\mathrm{R}_{\sigma}=C_{2}$ : $C_{2}\{2 \pi l / k\}=\{-2 \pi l / k\}=\{\pi+2 \pi l / k\}$. These two sets form the total of $2 k$ equivalent stationary points. The two corresponding trajectories are mapped into each other by $\mathrm{R}_{\sigma}$. In contrast, $\mathrm{R}_{\sigma}$ maps the even- $k$ set into itself.

\section{Behavior with respect to $R_{\lambda}$}

It is the $R_{\lambda}$ symmetry of the perpendicular orbit [and of our reduced problem with Hamilton function $\mathcal{J}_{\varepsilon}\left(\sigma, p_{\sigma}, \theta\right)$ where $R_{\lambda}$ corresponds to time reversal $\theta \rightarrow-\theta$ ] that gets broken in the even- $k$ case. To understand how this comes about consider

$$
\left(\begin{array}{cccc}
+ & - & + & - \\
a & b & c & d
\end{array}\right) \stackrel{\mathrm{R}_{\lambda}}{\rightarrow}\left(\begin{array}{cccc}
- & + & - & + \\
a & b & c & d
\end{array}\right),
$$

as opposed to

$$
\left(\begin{array}{ccc|ccc}
+ & - & + & - & + & - \\
a & b & c & b & c
\end{array}\right) \stackrel{\mathrm{R}_{\lambda}}{\rightarrow}\left(\begin{array}{ccc|ccc}
- & + & - & + & - & + \\
a & b & c & a & b & c
\end{array}\right) .
$$

In the even- $k$ case the $R_{\lambda}$ produces a new trajectory that goes in the opposite direction; in the odd- $k$ case $R_{\lambda}$ merely interchanges the two parts of the same trajectory leaving the whole trajectory invariant.

\section{Correspondence of trajectories and stationary points}

A $k$ bifurcation involves two period- $k$ orbits, stable and unstable, which correspond to $k$ stable and $k$ unstable stationary points of the reduced Hamilton function, such as the $C_{k}$ function in Table I. In our case any $k$ bifurcation also breaks one of the order-two a priori symmetries of the central orbit. This results in four period- $k$ orbits

$$
\begin{array}{cc}
\stackrel{\mathrm{R}_{\lambda}}{\Gamma_{0}^{+} \leftrightarrow \Gamma_{0}^{-},} \quad \Gamma_{1}^{+} \stackrel{\mathrm{R}_{\lambda}}{\leftrightarrow} \Gamma_{1}^{-} \quad \text { for even } k, \\
\stackrel{\mathrm{R}_{\sigma}}{\Gamma_{0} \leftrightarrow \Gamma_{2},} \quad \stackrel{\Gamma_{\sigma}}{\leftrightarrow} \Gamma_{3} \quad \text { for odd } k,
\end{array}
$$

created from (annihilated at) the perpendicular orbit. They correspond to stationary points $(69 \mathrm{~d})$ so that $\Gamma_{s}$ in (70) passes at $s(\pi / \kappa)+j(2 \pi n / k), j=0, \ldots, k-1$.

\section{Configuration-space images}

There are a few subtle details yet to be deduced. As follows from Eq. (67), coordinates of normal form (69b) are related to initial normal displacement $\sigma$ and momentum $p_{\sigma}$ so that at $\theta=\lambda=0$ the latter coincide with the standard rectangular frame for polar coordinates $(\sqrt{2 I}, \varphi)$. In other words, the contour plot of (69b) is (qualitatively) the same as the (interpolated) Poincaré surface of section with $\lambda=0, \sigma$ and $p_{\sigma}$ along horizontal and vertical axes, and $p_{\lambda}$ taking either sign (the $\pi$ map). If we now consider the points passed by $\Gamma_{s}$ in (70) we find that for equivalent orbits or for different passes of the same orbit the absolute values of $\left(\sigma, p_{\sigma}, p_{\lambda}\right)$ often are the same but the signs differ. Thus, in particular, orbits $\Gamma_{0,2}$ always pass through the origin $\lambda=\sigma=0$ $(\varphi= \pm \pi / 2)$, while $\Gamma_{1,3}$ never do. On the other hand, $\Gamma_{0}^{ \pm}$or $\Gamma_{1}^{ \pm}$pass there if $k \bmod 4$ equals 0 or 2. Furthermore, using the points $\varphi$, such that

\begin{tabular}{lllll} 
& \multicolumn{2}{c}{ Odd $k$} & \multicolumn{2}{c}{ Even $k$} \\
& $\Gamma_{0}, \Gamma_{2}$ & $\Gamma_{1}, \Gamma_{3}$ & $\Gamma_{0}^{+}, \Gamma_{0}^{-}$ & $\Gamma_{1}^{+}, \Gamma_{1}^{-}$ \\
\hline$|\varphi|$ & 0 & $\pi / 2$ & 0 & $\pi / k$ \\
$\sigma$ &,+- & 0 & + & + \\
$p_{\sigma}$ & 0 &,+- & 0 & \pm \\
$p_{\lambda}$ &,$\pm \mp$ &,$\pm \mp$ &,+- &,$\pm \mp$ \\
type & $\leftrightarrow$ & $\circlearrowleft$ & $\circlearrowleft$ & $\leftrightarrow$
\end{tabular}

we find that $\Gamma_{0,2}$ or $\Gamma_{1}^{ \pm}$pass such points in both directions, while $\Gamma_{1,3}$ and $\Gamma_{0}^{ \pm}$run in opposite directions. Therefore the configuration-space images of the former are degenerated (type " "w ") whereas those of the latter coincide (type " $\circlearrowleft$ ") and have opposite directions. In the following sections we display the configuration-space images of the actual trajectories. When verifying their symmetries remember to regard orbits of type " $\circlearrowleft$ " as directed curves. In Figs. 13, 15, and 19 we also distinguish the two equivalent-by-symmetry curves that either are halves of the same $\circlearrowleft$ trajectory or two $\leftrightarrow$ trajectories. 
[1] H. Poincaré, Les Méthodes Nouvelles de la Mécanique Céleste, Vol. III (Gauthier-Villars, Paris, 1892; Albert Blanchard, Paris, 1987); New Methods of Celestial Mechanics, Vol. III (Dover, New York, 1957).

[2] M. C. Gutzwiller, J. Math. Phys. 8, 1979 (1967); 10, 1004 (1969); 11, 1791 (1970); 12, 343 (1971).

[3] M. C. Gutzwiller, Chaos in Classical and Quantum Mechanics, Interdisciplinary Applied Mathematics Vol. 1 (Springer, New York, 1991).

[4] M. L. Du and J. B. Delos, Phys. Rev. Lett. 58, 1731 (1987); Phys. Rev. A 38, 1896 (1988); 38, 1913 (1988).

[5] D. Wintgen, Phys. Rev. Lett. 58, 1589 (1987); D. Wintgen and H. Friedrich, Phys. Rev. A 36, 131 (1987).

[6] J.-M. Mao and J. B. Delos, Phys. Rev. A 45, 1746 (1992).

[7] J.-M. Mao, J. Shaw, and J. B. Delos, J. Stat. Phys. 68, 51 (1992).

[8] J. Main, G. Wiebusch, K. Welge, J. Shaw, and J. B. Delos, Phys. Rev. A 49, 847 (1994).

[9] K. R. Meyer, Trans. Am. Math. Soc. 149, 95 (1970); 154, 273 (1971). See also K. R. Meyer, in Multiparameter Bifurcation Theory, Contemporary Mathematics Series Vol. 56, edited by M. Golubitsky and J. Guckenheimer (American Mathematical Society, Providence, RI, 1986), p. 373.

[10] A brief account of this work is given by D. A. Sadovskií, J. A. Shaw, and J. B. Delos, Phys. Rev. Lett. 75, 2120 (1995).

[11] J. Shaw, Ph.D. thesis, College of William \& Mary, 1993. As we show in this paper the period- $1 C_{2}$-symmetric bifurcation of the perpendicular orbit is organized in the fashion similar to the $k=4$ and 6 cases [6].

[12] An extension of the generic theory [16] to symmetric systems was given by M. A. M. de Aguiar, C. P. Malta, M. Baranger, and K. T. R. Davies, Ann. Phys. (N.Y.) 180, 167 (1987); M. A. M. de Aguiar and C. P. Malta, Physica D 30, 413 (1988).

[13] H. Dulac, Bull. Soc. Math. France 40, 324 (1912).

[14] G. D. Birkhoff, Dynamical Systems (American Mathematical Society, New York, 1966).

[15] A. Deprit, Celest. Mech. 1, 12 (1969); J. Henrard, ibid. 3, 107 (1970).

[16] K. R. Meyer and G. R. Hall, Introduction to Hamiltonian Dynamical Systems and the N-Body Problem, Applied Mathematics Series Vol. 90 (Springer, New York, 1992).

[17] Vl. I. Arnol'd, Geometrical Methods of the Theory of Ordinary Differential Equations, translated by J. Szücs, English translation edited by M. Levi, Series of Comprehensive Studies in Mathematics Vol. 250 (Springer, New York, 1988). Original Russian edition: Dopolnitel'nye Glavy Teorii Obyknovennykh Differentsial'nyk̂h Uravnenii (Nauka, Moscow, 1978).

[18] M. Morse and S. S. Cairns, Critical Point Theory and Differential Topology (Academic, New York, 1969).

[19] In mathematics the term "generic" has a precise meaning. A generic subset of an appropriately defined set (of functions, mappings, etc.) has two important properties: it must be open and everywhere dense. These properties are closely related to structural stability; see Refs. [17], Chap. 3, [16], Chaps. VIIIA, p. 202, and [54]. For example, integrable systems are structurally unstable in the set of all dynamical systems $[1,14]$, because an infinitely small perturbation typically destroys integrability; these systems are also not dense, and hence they cannot be used to approximate all possible dynamic regimes.
[20] The name comes from the pitchforklike bifurcation diagram in the parameter space.

[21] K. Meyer told us about a number of instances where similar phenomena were observed. He stressed that these phenomena are not generic in the one-parameter theory. See also Table I, footnote (c) and remarks of de Aguiar et al. (Ref. [12]), pp. 188 and 200.

[22] B. I. Żhilinskií and I. M. Pavliçhenkov, Zh. Eksp. Teor. Fiz. 92, 387 (1987) [Sov. Phys. JETP 65, 221 (1987)]; Ann. Phys. (N.Y.) 184, 1 (1988). For a general concise discussion see B. I. Żhilinskií, Teoriȳa Sloẑhnykkh Molekulyarnyk̂kh Spektrov (Moscow University Press, Moscow, 1989), Appendix 2 (in Russian) (English title: Theory of Complex Molecular Spectra).

[23] Note that the local symmetry $\mathfrak{g}$ of the normal forms in Table I is in fact higher than indicated. The actual symmetry includes reflection: $D_{k}=C_{k} \otimes \sigma$. Cases with true $C_{k}$ symmetry are qualitatively the same, even diffeomorphic to the ones shown. [To visualize this slightly curve the coordinate frame, for instance, curve axis $q$ (vertical) in the $C_{1}$ and $C_{2}$ plots to break $\sigma: p \mapsto-p$.$] Apart from bringing nothing new the improper$ rotations $D_{k}$ are not symplectic and thus should not be considered in the Hamiltonian case (cf. Appendix A).

[24] In general, in many degrees of freedom $(N>2)$, a complex hyperbolic case with four eigenvalues $\lambda \neq \bar{\lambda} \neq \lambda^{-1} \neq \bar{\lambda}^{-1}$ off the unit circle also arises; see Ref. [16], Chap. II C, Lemma 7, and Chap. II D, Lemma 13 and [33], Sec. 7.3(5). However, this case is not required for the present theory of bifurcations of periodic orbits, because here we are interested in resonances between the motion along and normal to the orbit. The motion along the orbit, or the driving force, is described by an auxiliary oscillator with eigenvalues $\lambda_{1,2}= \pm i$ (Secs. VI B and VII A 5). In a generic one-parameter theory at any critical value of the parameter $\varepsilon_{\text {crit }}$ only one transversal mode with eigenvalues $\lambda$ can be in such a resonance. It follows that $\lambda$ 's are purely imaginary: $\lambda=\exp ( \pm i 2 \pi n / k)$. Therefore, in this paper we only consider situations (7a) and (7b).

[25] A classical example of such generic parametrization is the Mathieu-Hill equation, where any of the two roots of $\omega(\varepsilon)=0$ are separated by finite intervals (Lyapunov's oscillation theorem (Ref. [37], Sec. 2.1)).

[26] At $\varepsilon_{\text {crit }}$ the monodromy matrix has at least one pair of multipliers (7b) and a corresponding Jordan block $\left(\begin{array}{ll}1 & \beta \\ 0 & 1\end{array}\right)$. It follows that members in a generic one-parameter family $M_{\varepsilon}$ of matrices, whose multipliers are of type (7a) [24], can have at most one degenerate pair (7b) ([17], Chap. 6, Sec. 30E). However, in a generic one-parameter family of $N \times N$ Hamiltonian matrices, another case can arise: there may be irreducible $4 \times 4$ blocks. We consider only the $2 \times 2$ case [24].

[27] In some exotic cases with very high symmetry $\mathfrak{G}$ present in the $\left(\sigma, p_{\sigma}\right)$ space transversal to the PO, there can be $\sigma_{1}$ and $\sigma_{2}$, which transform according to rows of the same irreducible representation, so that $\lambda_{1} \equiv \lambda_{2}$ and a $4 \mathrm{D}$ space $\sigma_{1,2}, p_{1,2}$ would have to be used.

[28] The idea of Żhilinskií and Pavlichenkov [22] is to classify bifurcations of the fixed points (equilibria) of generic onedegree-of-freedom Hamiltonians with possible a priori symmetries, and to study quantum and classical manifestations of these bifurcations. Their initial work [Ann. Phys. (N.Y.) 184, 1 (1988)] from where we cite our Table I deals with molecular rotation separated from vibration and electronic motion due to 
the Born-Oppenheimer principle; separation of vibrational modes of a molecule can be approximately achieved near the equilibrium configuration where the perturbation technique is valid [55]. In both cases symmetry enters as the a priori symmetry of the (equilibrium configuration of the) molecule. More generally this subject is discussed in [56]. As follows from our paper the range of applications of this theory is significantly broader.

[29] Vl. I. Arnol'd, Mathematical Methods of Classical Mechanics, translated by K. Vogtmann and A. Weinstein (Springer, New York, 1989). Original Russian edition: Matematicheskie Metody Klassicheskoí Mek̂khaniki (Nauka, Moscow, 1974).

[30] The transformation $(q, p) \rightarrow\left(z_{1}, z_{2}\right)$ is said to be symplectic with multiplier $2 i$, and since time is kept real, every Hamilton function has to be multiplied by $2 i$ to preserve the canonical structure. For example, $\left(p^{2}+q^{2}\right)=z \bar{z}$ must be replaced by $2 i z \bar{z}$, and then that factor carries through everywhere. This brings complex and symplectic structures into agreement.

[31] This "trick" has been suggested to us by K. Meyer [see K. R. Meyer and D. S. Schmidt, Funkcialaj Ekvacioj 20, 171 (1977), Eq. (3.16)]; cf. G. E. O. Giacaglia, Perturbation Methods in Nonlinear Systems, Applied Mathematics Sciences Vol. 8 (Springer, New York, 1972), Chap. 11.8.

[32] One common definition is worth remembering. By symmetry of a (Hamilton) function we understand the invariance of this function with respect to certain transformation of coordinates $\mathfrak{g}$; when we say that a map or flow is symmetric we mean that this map or flow, itself a transformation, commutes with $\mathfrak{g}$.

[33] Vl. I. Arnol'd, V. V. Kozlov, and A. I. Neishtadt, Mathematical Aspects of Classical and Celestial Mechanics, Dynamical Systems III, Encyclopedia of Mathematical Sciences Vol. 3 (Springer, New York, 1988).

[34] In a simple nearly linear case $\left[H_{\varepsilon} \sim J+\frac{1}{2}\left(p_{\sigma}^{2}+\sigma^{2}\right)+\cdots\right]$ and for $N=2$ Eq. (14a) defines a paraboloid. Since our study is local, $\sigma$ and $p_{\sigma}$ (in other words $\delta J$ ) can be made arbitrarily small [consider the area near the top of the paraboloid and its projection on the $\left(p_{\sigma}, \sigma\right)$ space] to suffice $(14 \mathrm{c})$ as well as curvature constraint (B2d), so that by the implicit function theorem we can always rewrite Eq. (14a) as Eq. (14b).

[35] Projections of periodic orbits on the configuration space can be qualitatively different. If the configuration-space image of an orbit is a closed curve (that possibly crosses itself) we call such orbit "circular." The image of a "degenerate" orbit degenerates into a line. A circular orbit runs in one distinct direction along its configuration-space image; it shares this image with another orbit running in the opposite direction. Therefore, circular orbits are not invariant with respect to reversing time. A circular orbit does not touch the border of the classically allowed domain of the configuration space. A degenerate orbit arrives at this border at the right angle, turns, and retraces itself. Since degenerate orbits run along their images in both directions they are time-reversal invariant.

[36] Handbook of Mathematical Functions, edited by M. Abramowitz and I. Stegun (GPO, Washington, DC, 1972), Chap. 20.

[37] W. Magnus and S. Winkler, Hill's Equation, Interscience Tracts in Pure and Applied Mathematics Vol. 20 (Wiley, New York, 1966).

[38] If the logarithm of the monodromy matrix $\ln M$ cannot be chosen real (and unique) we can deduce the real case from the initial problem by doubling the period, i.e., by considering
$M(4 \pi)=M^{2}$ whose multipliers are positive.

[39] It is interesting to apply the theory of foliations of threedimensional constant energy level sets of integrable systems in Refs. [48], Pt. III and [57], Chap. 4.1 to the problem of topology of the reduced phase space near the periodic orbit. For a stable central orbit the local normal form in Eq. (69b) defines the set of tori characterized by action $I$ of the motion normal to the orbit. (Another representation is a "filled torus.") In other words $I$ is the (local) Bott integral for Liouville tori. See also Sec. VII B 2.

[40] S.-N. Chow and J. K. Hale, Methods of Bifurcation Theory, Grundlehren der mathematischen Wissenschaften (Foundations of Mathematical Sciences) Vol. 251 (Springer, New York, 1982).

[41] We use indices as in [16], Chap. VII A 2: a near identity transformation has no $w_{0}$.

[42] In various references, either $M$ in Eq. (52b) or $M^{\prime}$ are called the order of the resonance. In the Hamiltonian case (52) such $M^{\prime}$ equals $M-1$. Furthermore, when we consider the order of the resonance for a periodic orbit and we have added two auxiliary equations (48b), we do not sum over those $m_{j}$ 's in (52d) that correspond to the auxiliary eigenvalues $\lambda= \pm i$ (cf. Sec. VII A 5).

[43] In group theory the set of (equivalent-by-symmetry) objects mapped into each other by all operations of the group is called an orbit [56]. We do not use this terminology since the reader may confuse it with periodic orbit. [Thus the twin periodic orbits $\Gamma^{ \pm}$in (70) form one orbit of the group $C_{2}=\left\{I, R_{\lambda}\right\}$.] Interestingly, the use of the term orbit in both cases has essentially the same idea.

[44] R. T. Swimm and J. B. Delos, J. Chem. Phys. 71, 1706 (1979).

[45] M. Robnik, J. Phys. A 14, 3195 (1981); M. Robnik and E. Schrüfer, ibid. 18, L853 (1985).

[46] K. D. Krantzman and D. Farrelly, Phys. Rev. A 43, 1666 (1991); K. D. Krantzman, J. A. Milligan, and D. Farrelly, ibid. 45, 3093 (1992).

[47] M. W. Beims and G. Alber, Phys. Rev. A 48, 3123 (1993); J. Gao and J. B. Delos, Phys. Rev. A 49, 869 (1994).

[48] B. A. Dubrovin, A. T. Fomenko, and S. P. Novikov, Modern Geometry-Methods and Applications, translated by R. G. Burns, Springer Series in Soviet Mathematics, Graduate Texts in Mathematics Vol. 93 (Springer, New York, 1984). Original Russian edition: Sovremennaỹa Geometriya: Metody $i$ Priloẑheniỹa (Nauka, Moscow, 1979).

[49] J. B. Fraleigh, Calculus with Analytic Geometry (AddisonWesley, Reading, MA, 1980).

[50] J. B. Delos, S. K. Knudson, and D. W. Noid, Phys. Rev. A 28 , 7 (1983); 28, 1208 (1983). Note that the scaling constant used by Mao and Delos for $\rho$ in Ref. [6] differs by $1 / 2$ :

$$
\hat{\rho}=\alpha \rho, \quad \hat{z}=\alpha z, \quad \alpha=\frac{1}{2}(B / c)^{2 / 3}, \quad \hat{S}=\frac{1}{2}(B / c)^{-1 / 3} .
$$

[51] Even though the topology of the original DKP phase space is quite different from the topology of the $(u, v)[$ or $(\rho, z)]$ representation, we can make the $(u, v)$ dynamics equivalent to that in the original DKP using special Maslov indices: whenever the electron passes through $v$ or $u$ axes the Maslov index increases by 1 [7].

[52] Alternatively, the Coulomb singularity can be removed by full 3D KS transformation, see E. L. Stiefel and G. Scheifele, Linear and Regular Celestial Mechanics, Ser. Grundlehren der mathematischen Wissenschaften (Foundations of Mathematical Sciences) Vol. 174 (Springer, Berlin, 1970), and Ref. [46]. 
[53] Ch. Cerjan and W. P. Reinhardt, J. Chem. Phys. 71, 1819 (1979); W. P. Reinhardt and D. Farrelly, J. Phys. (Paris), Colloq. 43, C2-29 (1982); C. Jaffé and W. P. Reinhardt, J. Chem. Phys. 77, 5191 (1982).

[54] T. Poston and I. Stewart, Catastrophe Theory and its Applications (Pitman, London, 1978); R. Gilmore, Catastrophe Theory for Scientists and Engineers (Wiley, New York, 1981);
Vl. I. Arnol'd, Catastrophe Theory, translated by R. Thomas (Springer, New York, 1984).

[55] D. A. Sadovskií, N. G. Fulton, J. R. Henderson, J. Tennyson, and B. I. Żhilinskií, J. Chem. Phys. 99, 906 (1993).

[56] D. A. Sadovskií and B. I. Žhilinskií, Phys. Rev. A 47, 2653 (1993); 48, 1035 (1993).

[57] A. T. Fomenko, Simplecticheskaȳa Geometriỹa (Moscow University Press, Moscow, 1988) (in Russian). 